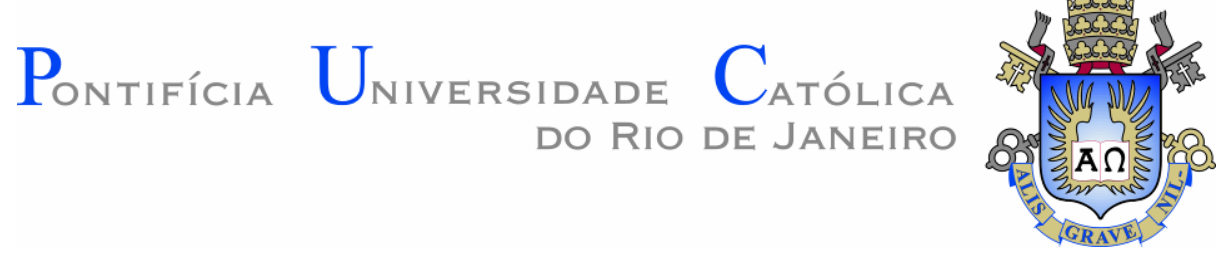

Luis Diego Valdez Silva

Slot Coating of Particle Suspension

Thesis presented to the Programa de Pós-Graduação em Engenharia Mecânica of the Departamento de Engenharia Mecânica do Centro Técnico Científico da PUC-Rio, as partial fulfillment of the requirements for the degree of Mestre.

Advisor : $\quad$ Prof. Márcio da Silveira Carvalho

Co-Advisor : $\quad$ Prof. Diego Martín Campana

Rio de Janeiro

April 2013 


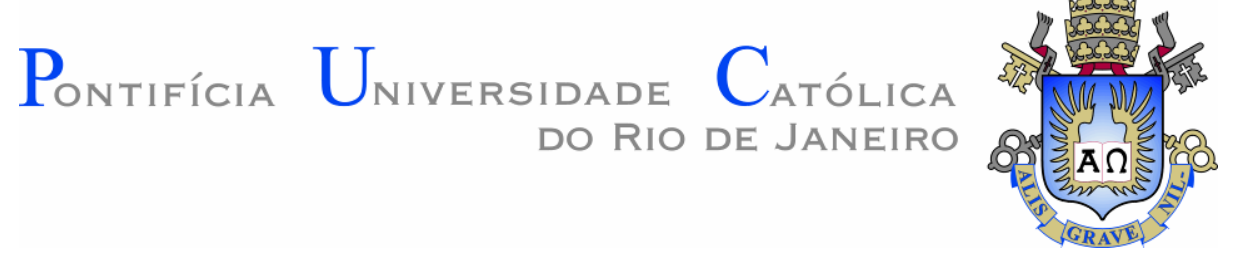

Luis Diego Valdez Silva

\title{
Slot Coating of Particle Suspension
}

Thesis presented to the Programa de Pós-Graduação em Engenharia Mecânica of the Departamento de Engenharia Mecânica do Centro Técnico Científico da PUC-Rio, as partial fulfillment of the requirements for the degree of Mestre.

\author{
Prof. Márcio da Silveira Carvalho \\ Advisor \\ Departamento de Engenharia Mecânica - PUC-Rio \\ Dr. Diego Martín Campana \\ Co-Advisor \\ Conicet - INTEC
}

Profa. Angela Ourivio Nieckele

Departamento de Engenharia Mecânica - PUC-Rio

Prof. Paulo Roberto de Souza Mendes

Departamento de Engenharia Mecânica - PUC-Rio

Prof. José Eugenio Leal Coordinator of the Centro Técnico Científico - PUC-Rio 
All rights reserved.

\section{Luis Diego Valdez Silva}

Graduated in Mechanical Engineering from Universidad

Nacional de Trujillo in September 2009.

Ficha Catalográfica

Silva, Luis Diego Valdez

Slot coating of particle suspension / Luis Diego Valdez Silva; advisor: Márcio da Silveira Carvalho ; coadvisor: Diego Martín Campana. - 2013.

137 f. : il. (color); $30 \mathrm{~cm}$

1. Dissertação (mestrado)-Pontifícia Universidade Católica do Rio de Janeiro, Departamento de Engenharia Mecânica, 2013.

Inclui bibliografia

1. Engenharia mecânica - Teses. 2. Processo de revestimento. 3. Suspensão de partículas. 4. Método dos elementos finitos. 5. Migração de partículas. I. Carvalho, Márcio da Silveira. II. Martín Campana, Diego. III. Pontifícia Universidade Católica do Rio de Janeiro. Departamento de Engenharia Mecânica. IV. Título. 


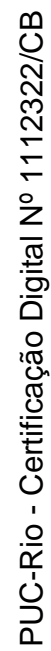

To my family. 


\section{Acknowledgments}

I would like to thank my advisor, Professor Márcio da Silveira Carvalho, for all the support and advices during the development of this work.

I would like to thank my co-advisor, Professor Diego Martín Campana, for the discussions about the problems involved in this work and the helpful advices.

I shall thank CAPES and FAPERJ for the financial support.

My acknowledgments to my colleges of the "Laboratório de Micro Hidrodinâmica e Meios Porosos" of the PUC-Rio.

I extend my gratitude to Luis Quevedo, José Olivencia and Giovanni Zucchi, my professors in Peru.

I would like to thank my family for all their constant support. 


\section{Abstract}

Valdez Silva, Luis Diego; Carvalho, Márcio da Silveira (Advisor); Campana, Diego Martín (Co-Advisor). Slot coating of particle suspension. Rio de Janeiro, 2013. 137p. Msc. Dissertation - Departamento de Engenharia Mecânica, Pontifícia Universidade Católica do Rio de Janeiro.

Slot coating process is commonly used in the manufacture of high technology products such as flexible circuits, LCD and OLED displays, among others. The thickness of the coated liquid film depends on the flow rate and substrate velocity. It is very important to study the flow in the coating bead region, because the flow pattern has a strong effect on the quality of the coated film. Most of the available studies on slot coating consider the fluid as a Newtonian liquid. However, coating liquids are usually polymer solutions or particle suspensions. The effect of dissolved polymer molecules in the flow has been analyzed recently. However, the effect of suspended particle remains unknown. The goal of this work is to develop a fundamental understanding of the effect of suspended particles in the flow pattern in a slot coating process. The local changes in particle concentration can lead to great changes in the local liquid viscosity and consequently in the flow and process limits. In this research, the particle suspension is a system composed by a Newtonian liquid as a continuous phase and rigid spherical particles as the dispersed phase. The particle transport equations takes into account the diffusion due to gradients of concentration, viscosity and deformation rates. The particle transport equation together with the mass and momentum conservation equations are used to describe the twodimensional free surface flow. These equations are solved using the Galerkin/Finite element method. The numerical code was validated by comparing numerical prediction obtained in circular tube flow with the available exact solution. The results obtained in slot coating flow show regions of high and low concentration of particles and its effect of the flow, and the variation of particle concentration in the deposited thin film. 


\section{Keywords}

Slot coating process, particle suspension, finite element method, particle migration. 


\section{Resumo}

Valdez Silva, Luis Diego; Carvalho, Márcio da Silveira; Campana, Diego Martín. Análise do Processo de Revestimento de Suspensões de Partículas. Rio de Janeiro, 2013. 137p. Dissertação de Mestrado Departamento de Engenharia Mecânica, Pontifícia Universidade Católica do Rio de Janeiro.

O processo de revestimento por extrusão é muito usado na fabricação de produtos de alta tecnologia tais como circuitos flexíveis, telas LCD e OLED, dentre outros. A espessura do filme revestido depende da vazão de alimentação e da velocidade do substrato. $\mathrm{O}$ estudo do escoamento na região de aplicação é de muita importância, devido à influência que tem sobre a qualidade do filme revestido. A grande maioria dos trabalhos disponíveis na literatura consideram o líquido como um fluido Newtoniano. Porém, nos processos de revestimentos nas indústrias, os líquidos comumente usados são soluções poliméricas e suspensões de partículas. O efeito da presença de moléculas poliméricas dissolvidas no fluido de trabalho nos padrões de escoamento e consequentemente na janela de operação do processo foi estudado recentemente, contudo, o efeito de partículas suspensas no escoamento é ainda desconhecido. O objetivo deste trabalho é compreender melhor a influência da suspensão de partículas no padrão de escoamento que ocorre na região de aplicação de um filme fino de líquido sobre um substrato em movimento. A variação local da concentração de partículas no líquido pode levar a grande variações na viscosidade e consequentemente mudanças no escoamento e nos limites do processo. Neste estudo, considera-se a suspensão como um sistema formado por um fluido Newtoniano como a fase contínua e partículas esféricas rígidas como a fase dispersa. A equação de transporte de partículas engloba os efeitos de difusão de partículas devido a gradientes de concentração, viscosidade e taxa de deformação. As equações de transporte de partículas em conjunto com as equações de conservação de massa e quantidade de movimento são usadas para descrever o escoamento bidimensional com fronteiras livres. Estas equações são resolvidas aproximadamente pelo método de Galerkin/Elementos Finitos. A validação do código numérico foi feita comparando com resultados analíticos 
disponíveis para escoamento em tubos de seção circular. Os resultados obtidos para o escoamento no precesso de revestimento por extrusão mostram as regiões de maior e menor concentração de partículas e como esta variação altera o escoamento, e a variação da concentração ao longo da espessura do filme depositado.

\section{Palavras-chave}

Processo de revestimento, suspensão de partículas, método dos elementos finitos, migração de partículas. 


\section{Contents}

1. Introduction 22

1.1. Coating process 22

1.2. Slot Coating Method 25

1.2.1. Coating Window 27

1.2.2. Flow in the Coating Bead Region 29

1.3. Suspension of Particles 32

1.4. Scope of the Research and Previous Works 37

2. Mathematical Formulation of Free Surface Flow of Particle Suspension $\quad 42$

2.1. Equation of Motion for Concentrated Suspensions 42

2.1.1. Governing Equations 46

2.1.2. Boundary Conditions for the Free Surface Problem 51

2.2. Solution of Free Surface Problems 56

2.2.1. Elliptic Mesh Generation 58

2.2.2. Boundary Conditions of Mesh Equations 59

3. Solution Method for the System of Differential Equations 62

3.1. Solution Method by the GFEM 62

3.2. Solution of the Nonlinear System of Algebraic Equations by $\begin{array}{ll}\text { Newton's Method } & 67\end{array}$

4. Validation 69

4.1. Flow of Suspension of Particles through a Cylindrical Tube 71

4.1.1. Solution for Mesh M1 73

4.1.2. Solution for Mesh M2 79

4.2. Flow of Suspension of Particles through a Rectangular Channel 83

4.3. Closure 87

5. Results 89

5.1. Overview $\quad 89$

5.2. Particle Migration in the Feed Slot 90 
5.3. General Features of Particle Concentration Field in Slot Coating Flow

5.4. Influence of Film Thickness on Particle Concentration 103

5.4.1. Analysis for $t=0.5 H_{0}$

5.4.2. Analysis when Recirculation Flow Starts 112

5.4.3. Analysis when Recirculation Flow is Present 120

5.5. Influence of Film Thickness on Coated Layer Concentration 128

6. Conclusions 130

6.1. Conclusions 130

6.2. Future Works 131

7. References

132 


\section{List of Figures}

Figure 1.1: Coat of a substrate by a single liquid layer.................................. 22

Figure 1.2: Fabrication process of coated substrates................................... 23

Figure 1.3: Most common coating methods: (a) slot coating, (b) slide coating,

(c) curtain coating, (d) knife coating, (e) forward roll coating and (f) reverse roll coating.

Figure 1.4: Important features of single-layer slot coating method and the nomenclature used. 25

Figure 1.5: Various die designs of slot coater, patents: (a) O'Brien, 1984,

(b) O'Brien, 1984, (c) Kageyama \& Yoshida, 1986,

(d) Kageyama \& Yoshida, 1986. 26

Figure 1.6: Coating window of slot coating process. 27

Figure 1.7: Studied geometries: (a) Ruschak (1976), (b) Higgins \& Scriven (1980), (c) pressure and shear driven flow in the coating bead. 30

Figure 1.8: Low-flow limit predicted using the viscocapillary model. 31

Figure 1.9: Classification of suspensions. 33

Figure 1.10: Creeping flow around a sphere. 33

Figure 1.11: Normalized suspension viscosity..... 36

Figure 1.12: "Phase diagram" for suspension rheology. Remade from Stickel \& Powell (2005).

Figure 2.1: Relevant variables involved in the slot coating process with suspension of particles. 44

Figure 2.2: Irreversible, two body collisions with (a) spatially varying shear rate, (b) constant viscosity and (c) spatially varying viscosity......... 50

Figure 2.3: Scheme of the division of the boundaries................................... 52

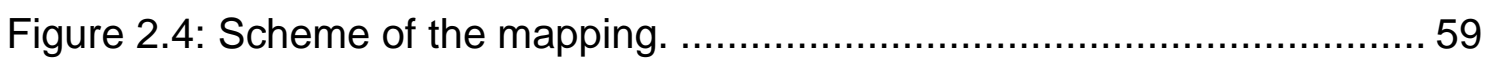

Figure 4.1: Cylindrical tube geometry and boundary conditions...................... 73

Figure 4.2: Evolution of particle concentration along the centerline. cbulk $=0.40$

Figure 4.3: Comparison of particle concentration: numerical vs. exact 
solutions. cbulk $=0.40$.

Figure 4.4: Developed velocity of the suspension at the end of the tube. cbulk $=0.40$.

Figure 4.5: Project "tubes4-006". 2D flow of suspension in a cylindrical tube. cbulk $=0.40$. 76

Figure 4.6: Evolution of particle concentration along the centerline. cbulk $=0.60$

Figure 4.7: Comparison of particle concentration: numerical vs. exact solutions. cbulk $=0.60$.

Figure 4.8: Developed velocity of the suspension at the end of the tube. cbulk $=0.60$

Figure 4.9: Project "tubes6-006". 2D flow of suspension in a cylindrical tube. cbulk $=0.60$.

Figure 4.10: Evolution of particle concentration along the centerline for the two meshes. cbulk $=0.40$.

Figure 4.11: Comparison of particle concentration: numerical vs. exact solutions. cbulk $=0.40$

Figure 4.12: Evolution of particle concentration along the centerline. cbulk $=0.60$

Figure 4.13: Comparison of particle concentration: numerical vs. exact solutions. cbulk $=0.60$.

Figure 4.14: Two-dimensional rectangular channel geometry and boundary conditions.

Figure 4.15: Evolution of particle concentration along the centerline. cbulk $=0.40$. 85

Figure 4.16: Comparison of particle concentration: numerical vs. exact solutions. cbulk $=0.40$.

Figure 4.17: Developed velocity of the suspension at the end of the tube. cbulk $=0.40$.

Figure 4.18: Project "tubes6-006". 2D flow of suspension in a cylindrical tube. cbulk $=0.40$. 86

Figure 5.1: Physical domain divided in 6 regions and mapped into a 
reference computational domain where the equations are solved.

Figure 5.2: Feed slot domain 91

Figure 5.3: Developed concentration at the exit of the slot, for different meshes. 92

Figure 5.4: Domain mesh. 93

Figure 5.5: Slot coating flow of suspension without migration.

Case "without.part.mig". 94

Figure 5.6: Slot coating flow of suspension with migration. Case "with.part.mig". 95

Figure 5.7: Feed slot region of case "with.part.mig". 96

Figure 5.8: Comparison of the pressure over the substrate in the upstream region 96

Figure 5.9: Upstream section of case "with.part.mig". 97

Figure 5.10: Magnified view of upstream flow. Case "with.part.mig". 97

Figure 5.11: Shorter upstream region. 98

Figure 5.12: Concentration at the downstream region. Case "with.part.mig". 99

Figure 5.13: Pressure over the substrate in downstream region. 100

Figure 5.14: (a) Velocity and (b) concentration (PHF) profiles at $\mathrm{x}=0.40 \mathrm{~mm}$. Case "with.part.mig". 101

Figure 5.15: Concentration at the downstream interface along interface length 102

Figure 5.16: Concentration across the developed coated layer...................... 102

Figure 5.17: Particle concentration across coated layer 103

Figure 5.18: Two-dimensional particle distribution at coating bead for

(a) $\mathrm{Ca}=0.10$, "slots4-017" and (b) $\mathrm{Ca}=1.0$, "slots4-015". 106

Figure 5.19: Velocity profile at $\mathrm{x}=0.40 \mathrm{~mm}$ for (a) Ca $=0.10$, "slots4-017" and (b) $\mathrm{Ca}=1.0$, "slots4-015".

Figure 5.20: Concentration profiles at $\mathrm{x}=0.40 \mathrm{~mm}$, for (a) $\mathrm{Ca}=0.10$,

"slots4-017" and (b) $\mathrm{Ca}=1.0$, "slots4-015". 107

Figure 5.21: Pressure over substrate for $\mathrm{Ca}=0.10$ 108

Figure 5.22: Pressure over substrate for $\mathrm{Ca}=1.0$. 108 
Figure 5.23: Concentration at free surface region for (a) $\mathrm{Ca}=0.10$,

"slots4-017" and (b) $\mathrm{Ca}=1.0$, "slots4-015".

Figure 5.24: Free surface shape for Newtonian liquid ("New.flow2")

and suspension ("slots4-015"), for $\mathrm{Ca}=1.0$.

Figure 5.25: Concentration along the interface length for $\mathrm{Ca}=0.10$

("slots4-017") and $\mathrm{Ca}=1.0$ ("slots4-015")

Figure 5.26: Particle concentration across coated layer for $\mathrm{Ca}=0.10$

("slots4-017") and $\mathrm{Ca}=1.0$ ("slots4-015")

Figure 5.27: Two-dimensional particle distribution at coating bead for

(a) $\mathrm{Ca}=0.1$ ("slots4-038") and (b) $\mathrm{Ca}=1.0$ ("slots4-060").

Figure 5.28: Velocity profile at $\mathrm{x}=0.40 \mathrm{~mm}$ for (a) $\mathrm{Ca}=0.1$ ("slots4-038")

and (b) $\mathrm{Ca}=1.0$ ("slots4-060").

Figure 5.29: Concentration profiles at $\mathrm{x}=0.40 \mathrm{~mm}$, for (a) $\mathrm{Ca}=0.1$

("slots4-038") and (b) $\mathrm{Ca}=1.0$ ("slots4-060").

Figure 5.30: Concentration distribution along downstream slot die lip for

$\mathrm{Ca}=0.1$ ("slots4-038") and $\mathrm{Ca}=1.0$ ("slots4-060"). 115

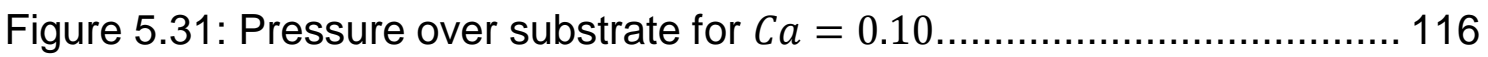

Figure 5.32: Pressure over substrate for $\mathrm{Ca}=1.0$

Figure 5.33: Concentration at free surface region for (a) $\mathrm{Ca}=0.1$

("slots4-038") and (b) $\mathrm{Ca}=1.0$ ("slots4-060").

Figure 5.34: Comparison of free surface shape for $\mathrm{Ca}=0.1$ of suspension flow ("slots4-038") and Newtonian flow ("New.flow3").

Figure 5.35: Comparison of free surface shape for $\mathrm{Ca}=1.0$ of suspension flow ("slots4-060") and Newtonian flow ("New.flow4").

Figure 5.36: Concentration along the interface length for $\mathrm{Ca}=0.1$

("slots4-038") and $\mathrm{Ca}=1.0$ ("slots4-060"). 119

Figure 5.37: Particle concentration across coated layer for $\mathrm{Ca}=0.1$

("slots4-038") and $\mathrm{Ca}=1.0$ ("slots4-060"). 120

Figure 5.38: Particles convected to coated layer top. 120

Figure 5.39: Two-dimensional particle distribution at coating bead for

$$
\mathrm{Ca}=0.1 \text { ("slots4-92"). }
$$

Figure 5.40: Closer view of recirculation region. 122

Figure 5.41: Streamlines for coated layer. 123 
Figure 5.42: (a) Velocity and (b) concentration profiles at $x=0.3 \mathrm{~mm} \ldots \ldots \ldots . .123$

Figure 5.43: Pressure over the substrate for case "slots4-092" .................... 124

Figure 5.44: Concentration at free surface region for case "slots4-092" ........ 125

Figure 5.45: Free surface shape for Newtonian liquid and suspension

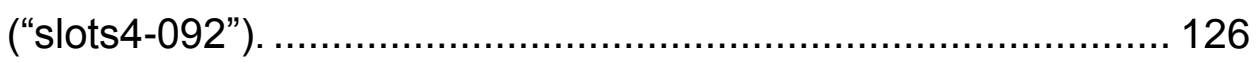

Figure 5.46: Concentration along interface length for case "slots4-092". ...... 126

Figure 5.47: Location of stagnation point for (a) suspension flow

("slots4-092") and (b) Newtonian flow.

Figure 5.48: Tangential velocity along the free surface for case "slots4092".

Figure 5.49: Particle concentration across coated layer for case

"slots4-092".

Figure 5.50: Fully developed concentration profile at different film thickness. 


\section{List of Tables}

Table 1.1: Dispersions classification. ....................................................... 32

Table 3.1: Degrees of freedom at each element. .................................... 67

Table 4.1: Meshes used in the tube simulation. ........................................... 73

Table 4.2: Data for the simulation for mesh $M 1$.................................... 74

Table 4.3: Data for the simulation for mesh M2 ....................................... 79

Table 4.4: Data for the simulation. Rectangular channel............................ 84

Table 5.1: Mesh uses in the feed slot suspension flow. .............................. 91

Table 5.2: Relevant variables for each case. .............................................. 91

Table 5.3: Number of elements in the mesh.......................................... 93

Table 5.4: Parameters for slot coating simulation for $t=0.5 H 0 \ldots \ldots \ldots \ldots \ldots . . . . .105$

Table 5.5: Parameters for slot coating simulation when recirculation flow

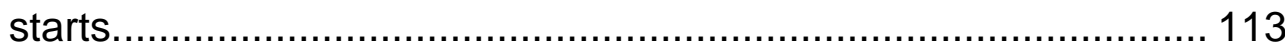

Table 5.6: Parameters for slot coating simulation when recirculation flow

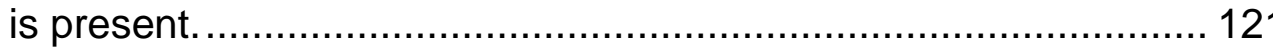




\section{List of Abbreviations}

$\begin{array}{ll}\text { 1D } & \text { One-Dimensional } \\ \text { 2D } & \text { Two-Dimensional } \\ \text { DCL } & \text { Dynamic Contact Line } \\ \text { DoF } & \text { Degrees of Freedom } \\ \text { GFEM } & \text { Galerkin Finite Element Method } \\ \text { LED } & \text { Light-Emitting Diodes } \\ \text { OLED } & \text { Organic Light-Emitting Diode } \\ \text { PDEs } & \text { Partial Differential Equations } \\ \text { PHF } & \text { Concentration Field } \\ \text { SCL } & \text { Static Contact Line }\end{array}$




\section{List of Symbols}

\begin{tabular}{|c|c|}
\hline$a$ & particle radius \\
\hline Bo & Bond number \\
\hline$c$ & particle concentration \\
\hline Ca & capillary number \\
\hline$c_{\text {buk }}$ & average or bulk particle concentration \\
\hline$c_{m}$ & maximum packing volume fraction of particles \\
\hline$c_{w}$ & particle concentration at the wall \\
\hline$\underline{D}$ & diffusion coefficient tensor of the inverse mapping equation \\
\hline$D_{\xi}$ & $\begin{array}{l}\text { diffusion coefficient of the coordinate } \xi \text { of the inverse } \\
\text { mapping equation }\end{array}$ \\
\hline $\begin{array}{l}D_{\eta} \\
\text { mapping }\end{array}$ & $\begin{array}{l}\text { diffusion coefficient of the coordinate } \eta \text { of the inverse } \\
\text { equation }\end{array}$ \\
\hline$g$ & acceleration of gravity \\
\hline$\underline{\underline{G}}$ & interpolated velocity gradient \\
\hline$H$ & half of the rectangular channel width \\
\hline$H_{0}$ & coating gap \\
\hline$H_{s}$ & feed slot gap \\
\hline $\begin{array}{l}\underline{i} \\
\text { domain }\end{array}$ & unit vector of the horizontal coordinate of the physical \\
\hline$\underline{I}$ & identity tensor \\
\hline II & second invariant of a tensor \\
\hline$\underline{j}$ & unit vector of the vertical coordinate of the physical domain \\
\hline$\underline{J}$ & Jacobian matrix of the Newton's iterative method \\
\hline$J_{T}$ & Jacobian determinant \\
\hline $\boldsymbol{J}_{T}$ & Jacobian matrix of the change of coordinates \\
\hline$k$ & Boltzmann's constant \\
\hline$k_{c}$ & migration coefficient due to spatially varying viscosity \\
\hline$k_{\eta}$ & migration coefficient due to spatially varying viscosity \\
\hline$n_{f}$ & unit vector normal to the free surface \\
\hline
\end{tabular}




\begin{tabular}{ll}
$n_{w}$ & unit vector normal to the wall \\
$N_{c}$ & particle flux due to spatially varying interaction frequency \\
$N_{\eta}$ & particle flux due to spatially varying viscosity \\
$N_{t}$ & total particle flux \\
$p$ & pressure \\
$P_{a t m}$ & atmospheric pressure \\
$P_{v a c}$ & vacuum pressure \\
Pe & Péclet number \\
$q$ & flow rate per unit width \\
$Q$ & flow rate \\
$r$ & radial coordinate \\
$R$ & radius of the cylindrical tube \\
$\hat{r}$ & dimensionless radius \\
$\underline{R}$ & vector of weighted residuals \\
$s$ & arc length \\
$t$ & film thickness \\
$T$ & absolute temperature \\
$\underline{T}$ & stress tensor \\
$t_{f}$ & unit vector tangent to the free surface \\
$t_{m i n}$ & minimum film thickness \\
$t_{w}$ & unit vector tangent to the wall \\
$u$ & component of suspension velocity in horizontal direction \\
$U_{m a x}$ & centerline velocity \\
$\underline{u}$ & suspension velocity \\
$v$ & component of suspension velocity in vertical direction \\
$V_{w}$ & substrate or web speed \\
$\underline{W}$ & weighting function vector of the momentum and \\
equations & \\
$x$ & horizontal coordinate of the physical domain \\
$x_{d c l}$ & horizontal coordinate of the dynamic contact line \\
& position vector in physical domain \\
\hline &
\end{tabular}




\section{Greek symbols}

$\beta$

$\dot{\gamma}$

slip coefficient

$\dot{\gamma}_{N L}$

shear rate or deformation rate

$\underline{\underline{\gamma}}$

non-local shear rate

rate-of-strain tensor

$\Gamma \quad$ boundary of the physical domain

$\bar{\Gamma}$

boundary of the reference domain

$\eta$

dynamic viscosity of the particle suspension

$\eta$

vertical coordinate of the reference domain

$\eta_{0}$

viscosity of the Newtonian suspending liquid

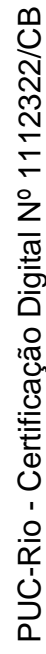

$\eta_{\mathrm{r}}$

relative suspension viscosity

dynamic contact angle

static contact angle

position vector in reference domain

horizontal coordinate of the reference domain

pi number

suspension density

surface tension

weighting function for the transport equation

Lagrangian biquadratic polynomial basis function

weighting function for the continuity equation

linear discontinuous basis function

$\underline{\underline{\tau}}$

viscous stress tensor

$\underline{\psi}$

weighting function for the interpolated velocity gradient

equation

$\psi_{j}$

Lagrangian bilinear polynomial basis function

$\Omega$

physical domain

$\bar{\Omega}$

reference domain 


\section{Introduction}

\section{1.}

\section{Coating process}

Coating process consists in the continuum deposition of a liquid film over a solid surface in motion, as sketched in Figure 1.1. The liquid displaces the gas phase existing over the surface. Therefore in this process three phases interact: solid, liquid and gas. This process is used to modify some properties of the coated surface.

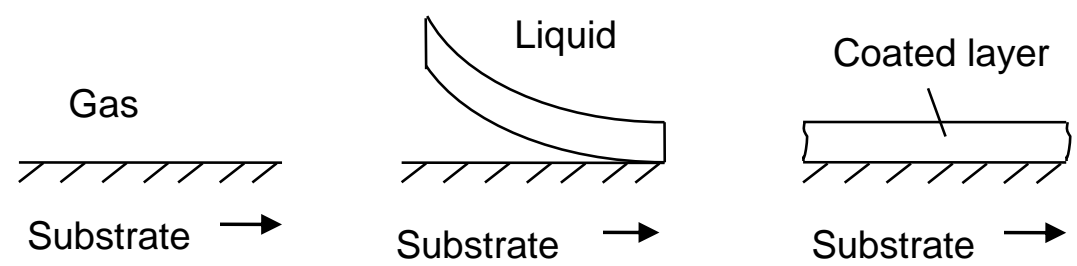

Figure 1.1: Coat of a substrate by a single liquid layer.

Coating process is used in the manufacturing of a wide variety of products, such as: imaging films, adhesive and magnetic tapes, magnetic and optic discs, flexible displays (LCD, OLED), etc. The thickness of the coated layer depends on the application, but generally this thickness is between 1 to 100 microns.

The solid surface to be coated is called substrate, and it could be flexible, porous and/or rigid; while the coating liquid could be a pure substance, a polymer solution, a colloidal dispersion, or a particle suspension.

A scheme of the diverse operations involved in coating processes is shown in Figure 1.2. The application of the liquid layer is done by one of the many existing methods. After the application of the liquid, it is necessary to solidify the coated layer. This is done by drying or curing. In the drying process the solvent is evaporated, leaving just the solid components over the substrate. The 
solidification by curing occurs when chemical reactions take place between the polymer chains.

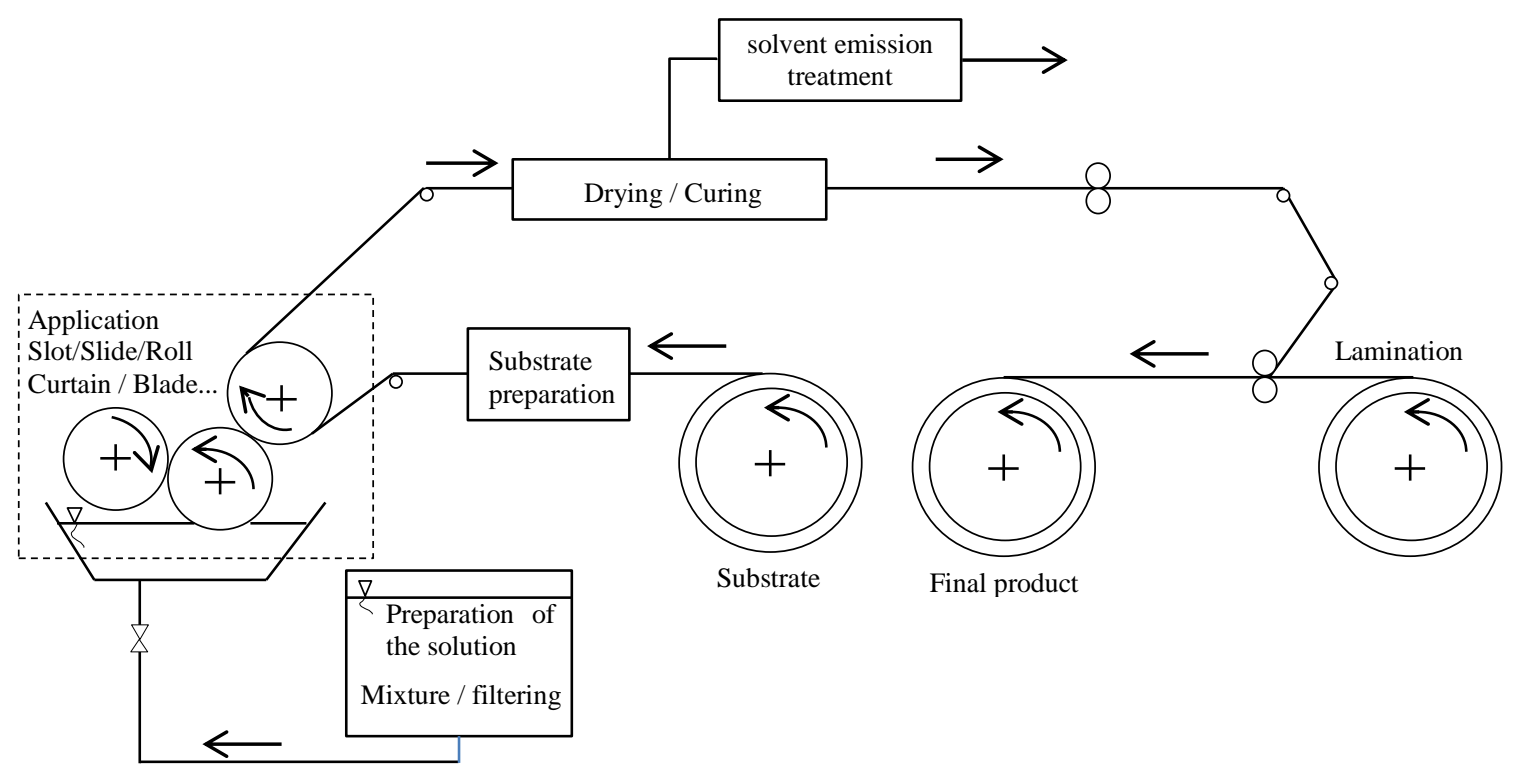

Figure 1.2: Fabrication process of coated substrates.

Coating process covers a multidisciplinary area and to have a better control of the process it is very important to fully understand the different phenomena that take place in each operation. In order to be competitive and to get products of higher quality it is important to understand the challenges involved in the coating process. The most important challenges are:

a) Thinner film layer;

b) Process optimization to minimize film thickness variation;

c) Faster production speed;

d) Use of more concentrated solutions;

e) Better understanding on how nano and micro particles flow inside the coating bead;

f) Better understanding on how nano and micro particles flow during drying.

Different coating methods have been developed. They can be classified in (i) pre-metered coating, where the thickness of the coated liquid layer is set by the prescribed flow rate, and in (ii) self-metered coating, in which the final wet 
thickness of the coated film is mostly controlled by the interactions of the fluid flow with the coating applicator, and the excess liquid applied to the substrate is removed.

The Figure 1.3 shows some examples of the most common coating methods used in industries: (a) slot coating, (b) slide coating, (c) curtain coating, (d) knife coating, (e) forward roll coating, (f) reverse roll coating. Additional coating methods are described in Kistler \& Schweizer (1997).

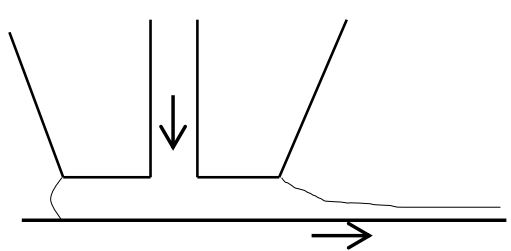

(a)

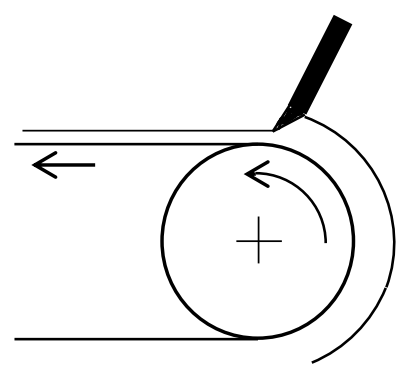

(d)

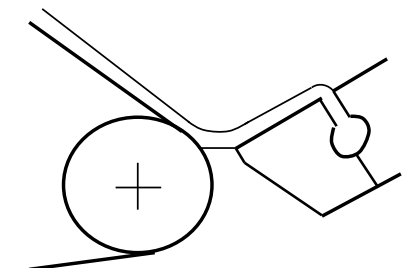

(b)

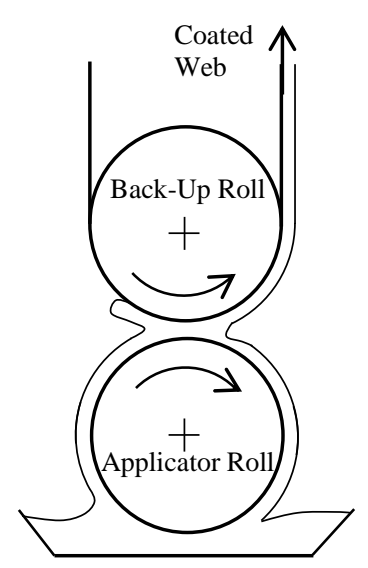

(e)

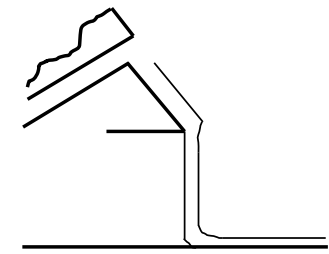

(c)

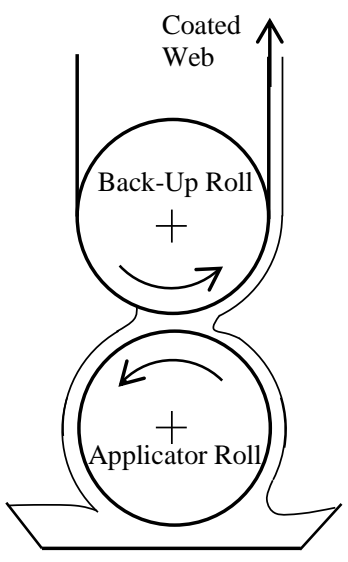

(f)

Figure 1.3: Most common coating methods: (a) slot coating, (b) slide coating, (c) curtain coating, (d) knife coating, (e) forward roll coating and (f) reverse roll coating.

The adequate selection of the coating method depends on many factors. The most important are:
a) Number of coated layers;
b) Final thickness of the coated liquid layer;
c) Liquid properties;
d) Film precision and uniformity; 
e) Velocity of the substrate (web velocity);

f) Substrate properties: porosity, flexibility, roughness, and/or smoothness.

\section{2.}

\section{Slot Coating Method}

One of the most efficient and widely used coating methods is the slot coating. This is a pre-metered method; where the thickness of the coated liquid layer is set by the prescribed flow rate fed into the coating die and it is independent of the process variables, making this class of method ideal for highprecision coating. In Figure 1.4 are shown some of the most important features of this method.

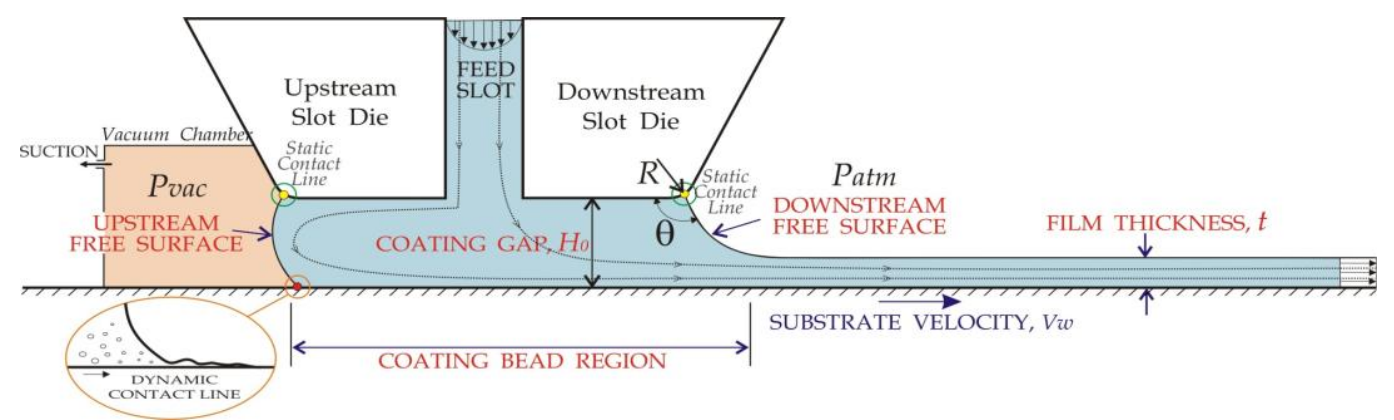

Figure 1.4: Important features of single-layer slot coating method and the nomenclature used.

In this process, the coating liquid is pumped to a coating die in which an elongated chamber distributes it across the width of a narrow slot through which the flow rate per unit width at the slot exit is made uniform. Exiting the slot, the liquid fills (wholly or partially) the gap between the adjacent die lips and the substrate translating rapidly past them.

The liquid in the gap, bounded upstream and downstream by gas-liquid interfaces or menisci, forms the coating bead as shown in Figure 1.4. The competition among viscous, capillary, pressure, and in some cases inertial and elastic forces, sets the range of operating parameters in which the viscous free surface flow of the liquid is two-dimensional and steady, which is the desired state. In order to sustain the coating bead at higher substrate speeds, Beguin (1954) proposed that a slight vacuum be applied to the upstream meniscus. 
The upstream and downstream menisci touch the slot lips at the static contact lines. But when the liquid touches the substrate, a dynamic contact line or wetting line is formed. The liquid-gas and solid interface forms an angle called apparent dynamic contact angle. At this line the liquid is continuously drag by the substrate in the downstream direction. Locally the fluid particle has a sudden change in velocity. The fluid flow in this region is still not well understood.

The nature of the flow in the coating bead, and therefore the uniformity of the liquid layer it delivers, can be affected by the substrate speed, the viscosity and any non-Newtonian properties of the liquid, and the configuration of the die lips immediately upstream and downstream of the slot exit.

There are variants in the configuration of the slot dies in this method. Different die lip configurations, inclinations of the slot, and number of coated layers are used in industry. Each type has its own application and many industries have their own technology. Kistler \& Schweizer (1997) present some of these configurations, as shown in Figure 1.5.

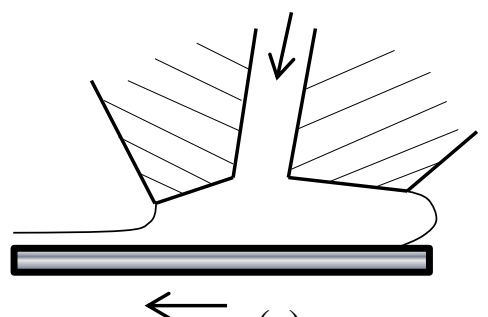

(a)

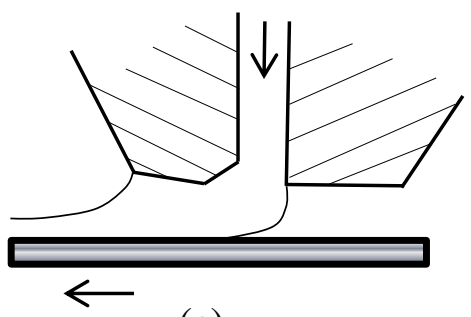

(c)

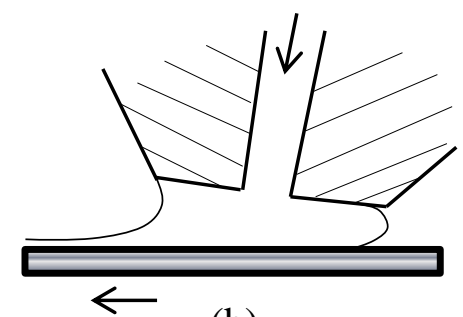

(b)

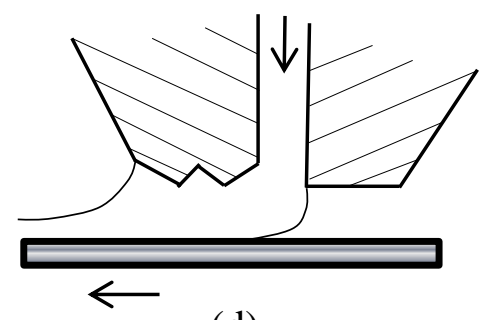

(d)

Figure 1.5: Various die designs of slot coater, patents: (a) O'Brien, 1984, (b) O'Brien, 1984, (c) Kageyama \& Yoshida, 1986, (d) Kageyama \& Yoshida, 1986. 


\subsection{1.}

\section{Coating Window}

The region in the space of operating parameters of a coating process where the delivered liquid layer is adequately uniform is usually referred to as the coating window. A good knowledge of the coating window for different coating methods is needed in order to predict whether a particular method can be used to coat a given substrate at a prescribed production rate. Figure 1.6 shows that the coating window is bounded by three modes of failure:

1) When the coated layer is thicker than the thinnest that can be produced at a fixed gap and substrate speed, i.e. $t>t_{\min }$ in Figure 1.6 , too great vacuum pressure at the upstream free surface causes liquid to be drawn along the die surface into the vacuum chamber. This diversion of liquid destroys the pre-metering condition.

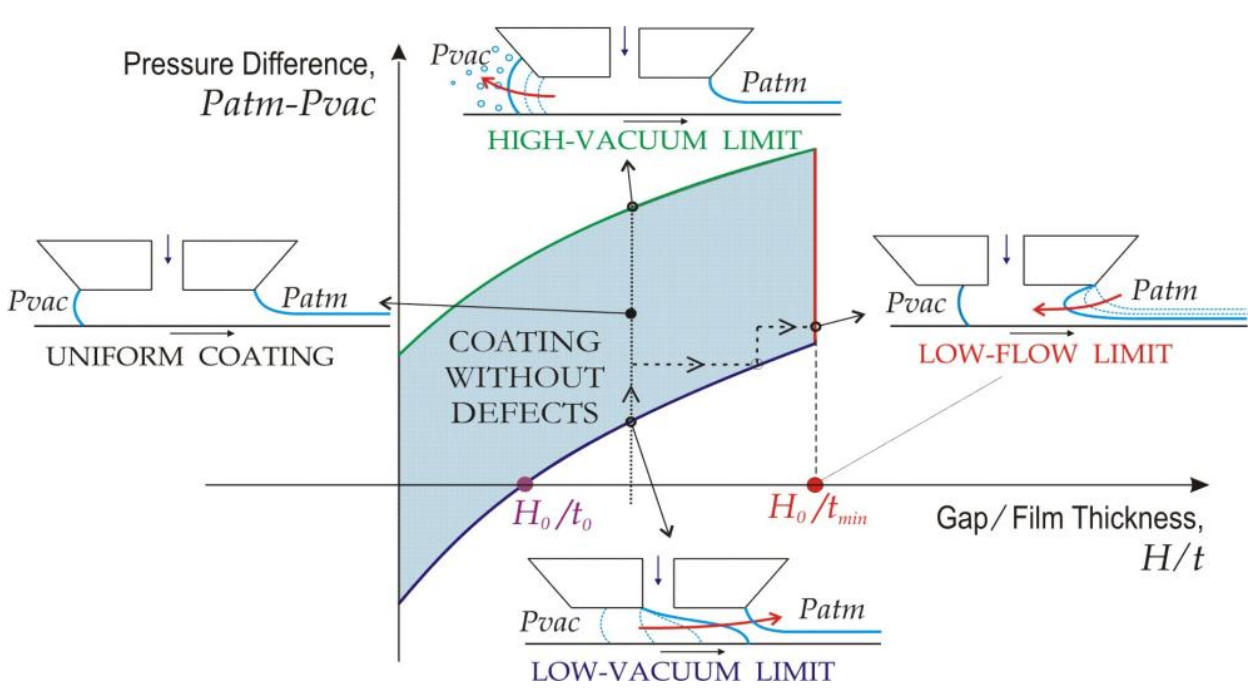

Figure 1.6: Coating window of slot coating process.

2) Too little vacuum at the upstream free surface leaves the net viscous drag force on the upstream part of the bead unbalanced by the pressure gradient that is imposed by capillary pressure forces in the menisci upstream and downstream and the difference in external pressure on those menisci (i.e. vacuum). In consequence the upstream meniscus shifts toward the feed slot until the bead drastically rearranges into a three-dimensional form that delivers separate rivulets to the substrate (Gates, 1999). As Figure 1.6 shows, 
the limit can be lowered by applying greater vacuum and thereby shifting the upstream meniscus away from the edge of the feed slot.

3) At given substrate speed, too little flow rate per unit width from the slot causes the downstream meniscus to curve so much that it cannot bridge the gap clearance $H_{0}$. Consequently, the meniscus loss its uniformity (becomes three-dimensional) and eventually it turns to be unstable, thus producing the breakup of the interface and forming separated rivulet and/or droplets of liquid deposited on the substrate. This transition from a continuous coated liquid layer is what is called here the low-flow limit: the minimum thickness of liquid that can be deposited from a gap of specified clearance at a given substrate speed. As Figure 1.6 depicts, it is independent of the vacuum applied, given that the vacuum is great enough to draw the upstream meniscus away from the feed slot. The outcome is the same when at a given flow rate per unit width from the slot, the substrate speed is too high. In this case, the low-flow limit is sometimes referred to as the high-speed limit. The outcome is essentially the same when at a given flow rate per unit width from the slot and a given substrate speed, the clearance of the downstream gap is too great. In this case, the low-flow limit is referred to as the wide-gap limit: the maximum gap from which a given thickness of liquid coating can be deposited on a substrate moving at specified speed.

Therefore, the uniformity of the coated film is not guaranteed by setting the flow rate. An acceptable coating layer depends on several other factors like:

a) Slot die configuration.

b) Properties of the liquid.

c) Operational parameters: coating gap, web velocity, vacuum pressure and flow rate. 


\subsection{2.}

\section{Flow in the Coating Bead Region}

Different studies were made to describe the flow in the coating bead. One of the first studies to determine the operation limit was developed by Ruschak (1976). In this model the upstream and downstream menisci are fixed to the corners of the slot, as shown in Figure 1.7-a. He extended the work of Landau and Levich (1942) assuming that the capillary forces are dominant, and the gravitational forces are negligible, therefore the capillary number $(C a=\eta V / \sigma)$ and the Bond number $\left(B o=g H^{2} \rho / \sigma\right)$ are very small. Hence, just the capillary forces determine the low-flow limit. He showed that the pressure drop across the downstream meniscus can be related to the film thickness, web speed, and liquid properties by:

$$
\Delta P=P_{0}-P_{1}=1.34(\mathrm{Ca})^{2 / 3} \frac{\sigma}{t}
$$

Then Higgins \& Scriven (1980) extended the work of Ruschak (1976) considering the viscous effects, but with the same hypothesis of low Capillary and Bond number. This model is also called the viscocapillary flow model. In this model, the upstream interface is free to move along the upstream lip, and the streamlines are rectilinear and parallel, in most part of the flow domain, as shown in Figure 1.7-b. The main flow under the die land is a combination of a Couette and Poiseuille contribution. Therefore, the total flow rate could be model as a result of the sum of a Couette flow rate, $q_{c}=V H / 2$, and Poiseuille flow rate, $q_{p}=(\Delta P / L) H^{3} / 12 \mu$, being the net flow zero, $q_{c}+q_{p}=0$, under the upstream die lip. Hence, there is a positive pressure gradient in this region, $P_{2}>P_{3}$, as shown in Figure 1.7-c. 


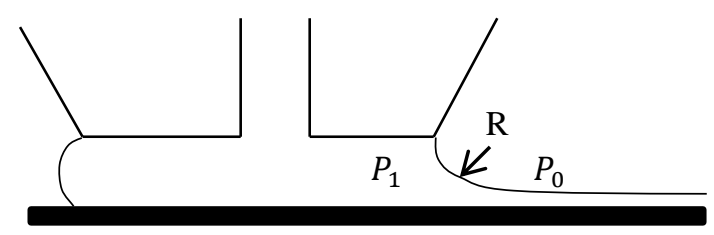

(a)

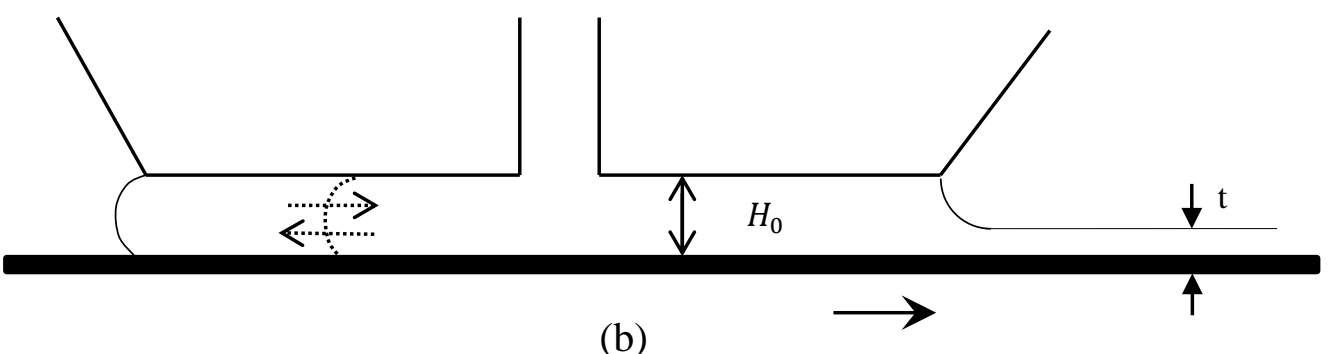

(b)

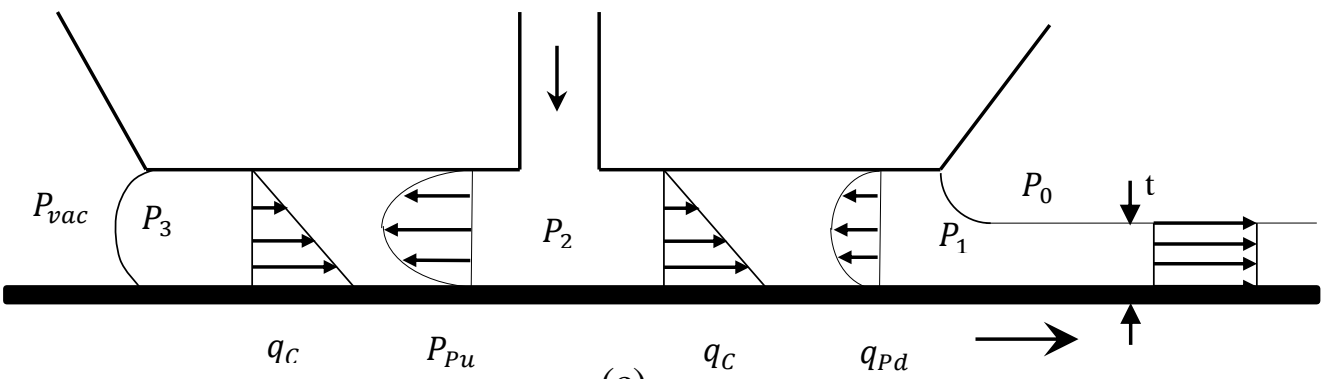

(c)

Figure 1.7: Studied geometries: (a) Ruschak (1976), (b) Higgins \& Scriven (1980), (c) pressure and shear driven flow in the coating bead.

According to the viscocapillary model, varying the flow rate $(q)$, hence the film thickness $(t)$, the pressure gradient has to change to adjust the Poiseuille flow contribution. Then, at a given substrate speed, the minimum flow rate per unit width (minimum liquid layer thickness, $t_{\min }$ ) is set by the greatest adverse pressure gradient that can be created in the downstream part of the gap. That arises when the upstream meniscus is hemicylindrical and situated just upstream of the feed slot, so that the pressure jump is the highest it can be, and the downstream meniscus is as curved as it can possibly be, but still connecting the free surface of the thin coated layer to the surface of the die lip. Using the YoungLaplace equation, the maximum pressure gradient at the interface, for minimum film thickness, is:

$$
\Delta P_{\text {max }}=\frac{2 \sigma}{H_{0}-t_{\text {min }}}
$$


Combining the equation 1.1 and equation 1.2 gives the relation of the operating parameters that set the low-flow limit:

$$
C a=\frac{1.82}{\left(\frac{H_{0}}{t_{\min }}-1\right)^{3 / 2}}
$$

This relation is plotted in Figure 1.8. Above the solid line, the coating bead is unstable and the two-dimensional steady flow does not exist. In experiments, crossing the low-flow limit from stable to unstable, the uniform coated layer gives way to alternating uncoated and coated stripes called rivulets. At a fixed coating gap, the minimum possible film thickness increases when the Capillary number increases, that is, higher production speeds require thicker wet layers.

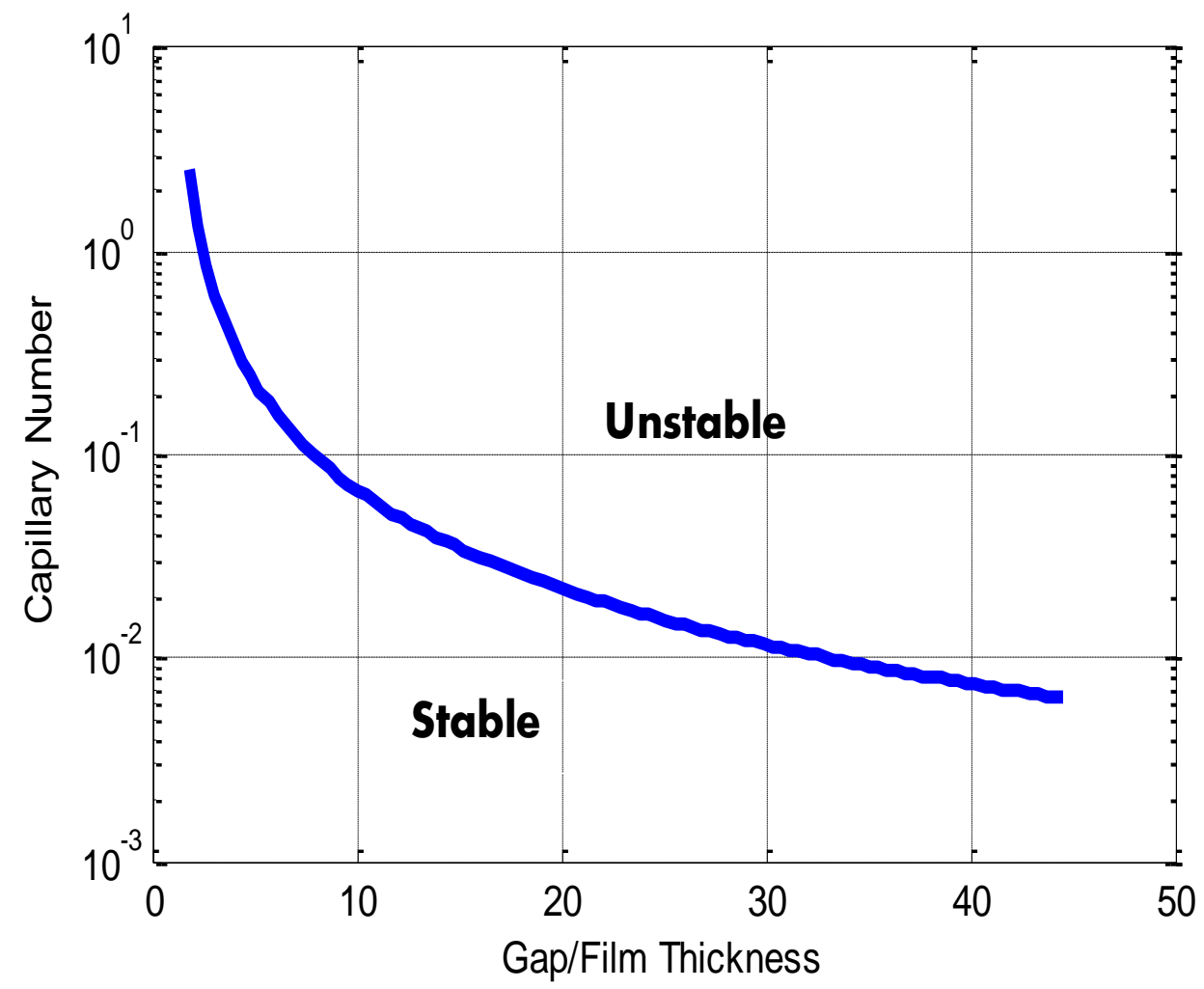

Figure 1.8: Low-flow limit predicted using the viscocapillary model. 


\section{3.}

Suspension of Particles

As it was mentioned before, several industries use polymers and suspension of particles as a coating liquid. In this research it was studied the flow of suspension of particles as a coating liquid. Many of the structured liquids that we come across in everyday life and industrial products are suspensions of particles in a liquid, with examples ranging from mud to blood, and custard to mustard. Other examples can be found in Barner (2000).

Suspensions belong to a general class of fluids, called dispersions. A dispersion is a system that results from the combination of gas, liquid, or solid phases. A classification could be seen in Table 1.1. From now on, we will be dealing with dispersion systems of "suspensions" type, more specifically, suspensions of mono-dispersed solid particles (all particles are of the same size and type) in a continuous liquid.

Table 1.1: Dispersions classification.

\begin{tabular}{|c|c|c|c|c|}
\cline { 3 - 5 } \multicolumn{2}{c|}{} & \multicolumn{3}{c|}{ Dispersed Phase } \\
\cline { 3 - 5 } \multicolumn{2}{c|}{} & Gas & Liquid & Solid \\
\hline \multirow{2}{*}{$\begin{array}{c}\text { Continuous } \\
\text { Phase }\end{array}$} & Gas & & vapors & aero-sol smokes \\
\cline { 2 - 5 } & Liquid & foams & emulsions & suspensions \\
\cline { 2 - 5 } & Solid & solid foams & & alloys, polymers \\
\hline
\end{tabular}

In turn, the suspensions are classified according to its shape and size, as shown in Figure 1.9. 


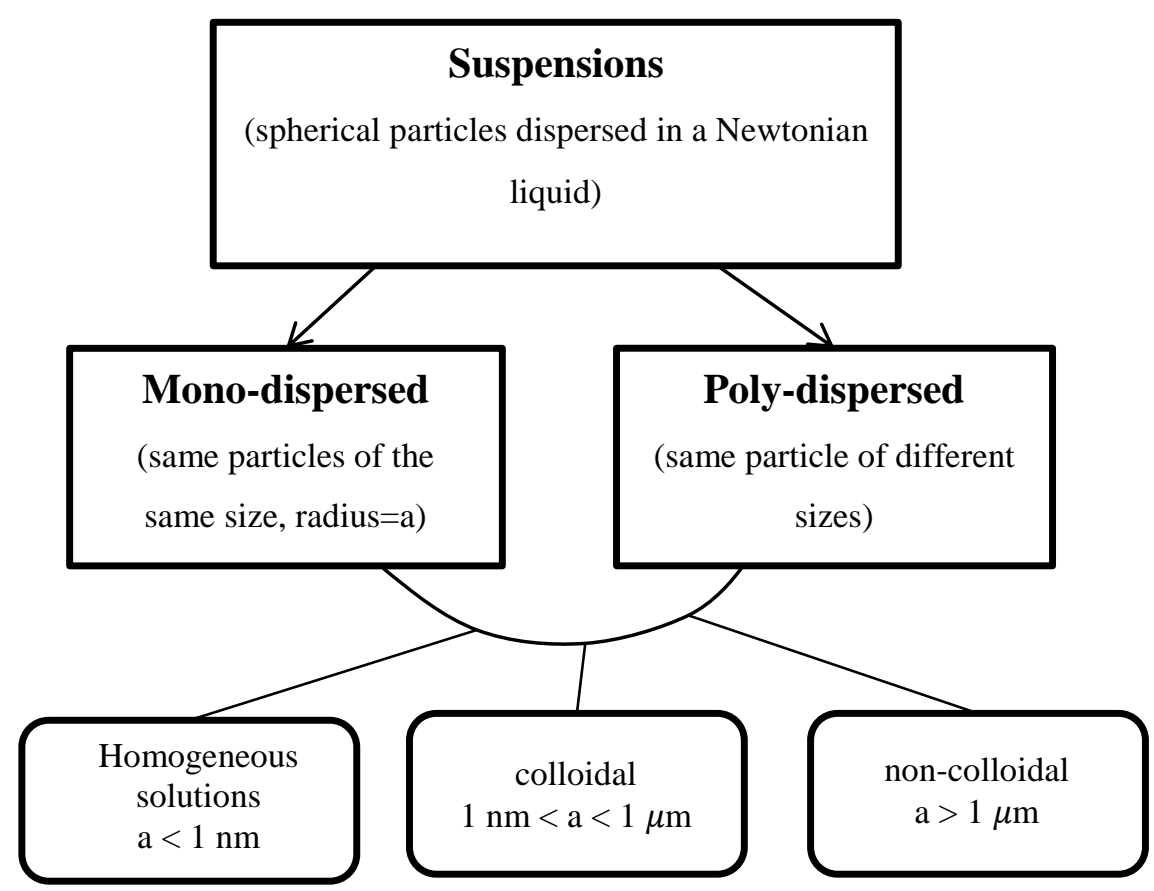

Figure 1.9: Classification of suspensions.

The addition of a rigid sphere to a liquid alters the flow field as illustrated in Figure 1.10. Extra energy dissipation will arise due to its presence, which will be reflected in a proportional increase in viscosity. This modifies the flow of the continuous phase. The main factors that alter the flow behavior are:

- The volumetric particle concentration in the liquid $(c)$, also known as the particle volume fraction. The suspension is said to be dilute usually if $c<0.05$, and dense or concentrated when $c \geq 0.30$.

- The particle distribution in the suspension. This affects the viscosity of the fluid, then the kinematic and dynamic of the flow.

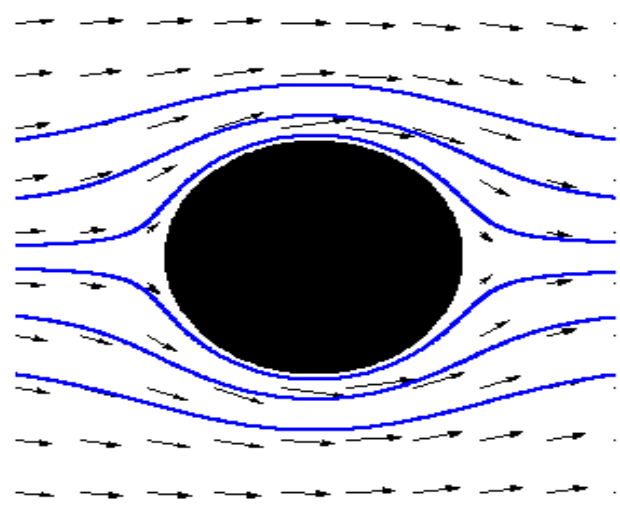

Figure 1.10: Creeping flow around a sphere. 
Forces of different origins exist and act on the particles inside a suspension. They depend on the particle size and shape. These include hydrodynamic interactions, electrostatic interactions, and other surface interactions that become important as surfaces come close together.

It will be defined some important dimensionless numbers that account the main forces acting on the suspension particles:

- Particle Reynolds number: ratio between inertial and viscous forces given by:

$$
R e_{p}=\frac{\rho_{f} \dot{\gamma} a^{2}}{\eta_{f}}
$$

where $\rho_{f}$ is the density of the continuous liquid phase, $\dot{\gamma}$ is the shear rate, $a$ is the particle radius, and $\eta_{f}$ is the continuous liquid phase viscosity.

- Péclet number: ratio between hydrodynamic and Brownian forces given by:

$$
P e=\frac{6 \pi \eta_{f} a^{2} \dot{\gamma}}{k T}
$$

where " $\mathrm{k}$ " is the Boltzmann's constant $\left[\mathrm{J} . \mathrm{K}^{-1}\right]$ and " $\mathrm{T}$ " is the absolute temperature $[\mathrm{K}]$.

These additional forces in the suspension alter the viscosity of the liquid, when compared to the viscosity of the continuous phase alone. Several models that relate the suspension viscosity and the particle volume fraction have been developed. In addition, these models take into account the viscosity of the Newtonian suspending liquid, $\eta_{0}$. To simplify the notation, from now on, it will be denoted:

$$
\eta_{r}=\frac{\eta}{\eta_{0}}
$$


as the relative suspension viscosity, where $\eta$ is the effective suspension viscosity.

The first model was proposed by Einstein (1906), which is valid for very dilute suspensions $(0 \leq c \leq 0.05)$. The Einstein law establishes a linear dependence between the particle volume fraction and the suspension viscosity.

$$
\eta_{r}=(1+2.5 c)
$$

Later, Batchelor extended the previous work of Einstein to a higher limit $(\phi \leq 0.15)$ providing a new suspension viscosity law.

$$
\eta_{r}=\left(1+2.5 c+K c^{2}\right)
$$

The term "K" accounts for hydrodynamic interactions between particle pairs that arise at higher volume fractions. Its values depend on the flow type.

Although the Einstein and Batchelor equations are important starting points, they give little help for real situations, since individual particles are not aware of one another's existence. Many empirical equations followed Eintein's exact mathematical effort, each of which sought to increase the concentration range into more practical region.

Later, researchers have measured and modeled the experimental data via various phenomenological equations that take into account the maximum possible packing volume fraction of particles, $c_{m}$. One of the more useful suspension viscosity models is the Krieger (1972) equation. This viscosity model is used in the present work, due to its extensive application in the suspension flow in different geometries with excellent results. This model, where $c_{m}$ is the maximum packing volume fraction of particles, is expressed as:

$$
\eta_{r}=\left(1-\frac{c}{c_{m}}\right)^{-2.67 c_{m}}
$$

Another model developed by Zarraga et al. (2000), has the convenient feature that obeys the Einstein relation in the dilute limit. 


$$
\eta_{r}=\frac{e^{-2.34 c}}{\left(1-\frac{c}{c_{m}}\right)^{3}}
$$

These models, that take into account the maximum packing fraction, have a singularity point when the particle volume fraction reaches the maximum value, as seen in Figure 1.11. The value of $c_{m}$ depends strongly on the shape and interaction, and it is found to be between 0.58 and 0.72 for monodispersed spheres.

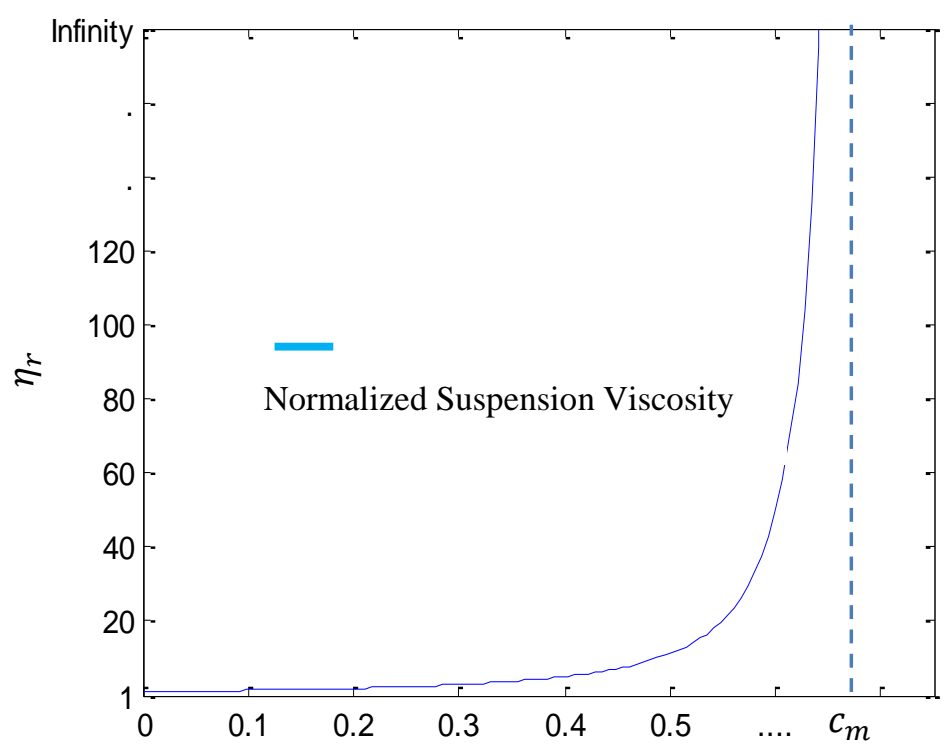

Figure 1.11: Normalized suspension viscosity.

Adding particles does not simply change the magnitude of the viscosity, but also can introduce all the known deviations from Newtonian behavior. Nevertheless non-colloidal (non-Brownian) suspensions, under some conditions, usually are considered as Newtonian fluids, because of the independence of viscosity from the shear rate. This assumption is only valid for intermediate shear rate values, as was demonstrated in the experiments made by Hoffman (1972). In addition, a dimensionless analysis shows that the suspension behavior depends on the dimensionless numbers $\mathrm{Re}$ and $\mathrm{Pe}$ (equation 1.4 and equation 1.5) as shown in Figure 1.12. 


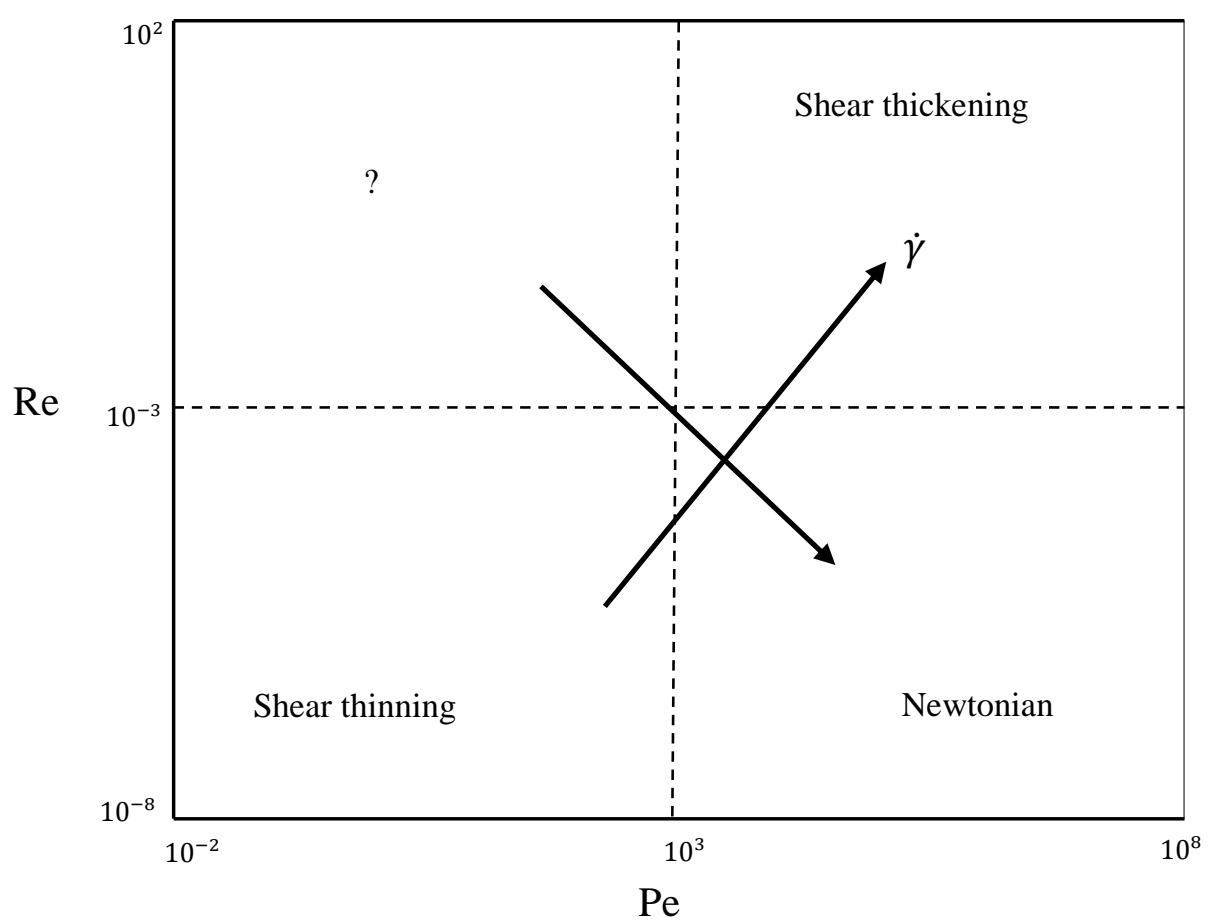

Figure 1.12: "Phase diagram" for suspension rheology. Remade from Stickel \& Powell (2005).

In conclusion, the flow description for a non-colloidal suspension is expressed by the stress tensor of a Newtonian fluid with viscosity that depends on the local volume fraction. It is also needed an equation that describes the migration of particles. This equation is the convection-diffusion equation for solid particles.

The use of particle suspension in coating process may lead to different flow patterns that should be studied, in order to improve both production rates and quality of the final coated product.

\section{4.}

\section{Scope of the Research and Previous Works}

The fundamental objective of this research is to predict the particle distribution in the coating bead and changes in the flow behavior in a single layer slot coating process. In addition, it is of fundamental interest to predict the particle concentration profile in the coated film.

The particles added to a Newtonian liquid modify the kinematic and dynamic behavior of the fluid. The properties of the suspension modify the 
velocity and pressure fields, the position and shape of the interfaces, and possibly the minimum wet thickness. The migration of particles and particle distribution is defined by the flow geometry and boundary conditions of the problem.

There are many theoretical models that quantify the migration of particles. Here, we have chosen the Phillips et al. (1992) constitutive model to describe the migration of particles in slot coating flow. The reason for this selection will be explained in the next chapter. This constitutive model accounts for particle migration due to both shear rate gradient and viscosity gradient.

This research covers two wide research areas: slot coating process and flow of particle suspension. A summary of the more relevant related works is presented next.

One of the first studies on the subject of slot coating was developed by Ruschak (1976). He used asymptotic expansion method for the flow in short slot die geometry. Gravitational and viscous forces were neglected. He obtained a relation between the pressure drop across the downstream meniscus and the film thickness, web speed and liquid properties, as shown in equation 1.1. Then, capillary pressure alone sets bounds on coating bead operability.

Then, Higgins \& Scriven (1980) extended the work of Ruschak (1976) by considering the viscous effects, but with low capillary (Ca) and Bond (Bo) number. The flow in the coating bead was modeled as the sum of a Couette flow and Poiseuille flow. They obtained new bounds on coating bead operability considering variable meniscus location.

Another study, done by Saito \& Scriven (1981), analyzed the influence of the flow rate $(\mathrm{Q})$, capillary and Reynolds $(\mathrm{Re})$ number to determine the position of the downstream meniscus of a Newtonian liquid using the Galerkin/Finite element method (GFEM). They extended the analysis of slot coating to higher capillary number and lower flow rates. Their results show clearly that, at constant Reynolds number, for high capillary number and low flow rate the meniscus invades into the slot (the separation angle diminishes) until it would have to make a separation angle that is lower than physically possible; i.e., the meniscus can no longer be attached to the slot.

Sartor (1990) presented a broad experimental and theoretical research related to single-layer slot coating process. He worked with a Newtonian liquid. In the experimental analysis, he presented the first visualizations of the flow for 
different slot die configurations and the influence of them in the coating window. In addition, he presented a map with several recirculation vortices inside the coating bead. In the theoretical analysis, it was shown that these vortices appear when the coated film is smaller than the third of the coating gap.

Gates (1999) also presented a wide experimental and theoretical research for Newtonian liquid in the slot coating process. He performed experimental analyses for different slot die configurations. Besides, it was shown different coating defects like rivulets and ribbing. This research showed that knife-edge configuration can extend the coating window.

Carvalho \& Kheshgi (2000) performed an experimental and theoretical study to obtain the low-flow limit in the slot coating process considering inertial effects, i.e. larger Reynolds number. They proved that at sufficiently high Reynolds and capillary numbers the minimum possible film thickness that can be coated decreases as web speed rises. It was concluded that the inertial effects extend the coating window.

The previous works presented above consider the flow of a Newtonian fluid, as a coating liquid, with constant viscosity. There are some studies of slot coating process with polymer solution as coating liquid. Lee et al. (2002) studied theoretically the Hele-Shaw flow, and the flow in the downstream region of the slot coating of viscoelastic fluids in the low metering rate limit. The viscoelastic fluid was modeled by the Oldroyd-B, FENE-CR, and FENE-P constitutive equations. Their numerical solution shown that viscoelasticity was found to increase the meniscus invasion in slot coating flows, and thus, reduce the separation angle at the static contact point.

Romero et al. (2004) examined both by theory and experiment the low-flow limit in slot coating of an extensional thickening polymer solution. They used an algebraic non-Newtonian constitutive equation that relates stress to the rate-ofstrain and relative-rate-of-rotation tensors. The results obtained revealed the same flow structure and stress field reported by previous work using differential constitutive models.

Concerning particle suspensions, there are a broad range of experimental and theoretical studies about their flow. The suspensions studied are usually concentrated suspensions of rigid monomodal spheres suspended in a Newtonian 
liquid. In all these studies, it is shown that there is a non-uniform distribution of particles in a suspension when there is an inhomogeneous shear rate field.

One of the first experimental studies on flow of particle suspensions was performed by Karnis et al. (1966). He showed that the suspension velocity profile in a tube deviates from the parabolic Newtonian solution. The velocity profile presents a blunting in the center of the tube, where there is a higher concentration of particles. In was concluded that the blunting of the profile depends on the concentration of particles and particle size, but is independent of flow rate and suspension viscosity. It was also observed that the particle translational velocity and the fluid translational velocity are the same. This work also showed that the suspension he used had a Newtonian behavior.

Gadala-Maria \& Acrivos (1980) performed an experimental research of particle migration at steady state in a Couette device. It was observed that the suspension viscosity decrease to an asymptotic value after long periods of shearing. Then Leighton \& Acrivos (1987a) demonstrated that the diminishing of the suspension viscosity, in the Gadala-Maria \& Acrivos (1980) experiment, is due to the shear-induced migration of particles out of the sheared Couette gap and into the fluid reservoir. In addition, their research proved that, even under creeping flow conditions, particles migrate across streamlines against concentration gradients when undergoing bounded shear flow. The migration occurs due to the surface roughness of particle-particle interactions. These interactions are irreversible because the particles do not return to their original streamlines. The research also proved that the particle diffusion due to shear rate gradient is proportional to the product of the square of particle radius and to the shear rate $\left(a^{2} \dot{\gamma}\right)$. Similar conclusions were obtained in experiments made by Abbott et al. (1991).

Phillips et al. (1992) extended the works of Leighton \& Acrivos (1987a, b) in the development of an equation that quantifies the flow-induced particle migration. It was proposed a phenomenological constitutive equation considering the viscosity-induced and shear-induced migration mechanisms. This model is also called the diffusive flux model. In section 2.1.1 of this text the two migration mechanisms considered in this model are described in detail. One of the advantages of this model is its simplicity of implementation. The present work uses the diffusive flux model to describe the migration of particles in the flow. 
Fang \& Phan-Thien (1995) performed numerical simulation of twodimensional particle migration of concentrated suspensions using the finite volume method. They used the Phillips et al. constitutive equation for particle flux. They performed simulations for steady and transient state of concentric and eccentric circular Couette flow. In the eccentric Couette flow, it was observed a recirculation flow, at large eccentricity ratios (greater than 0.5), that leads to a non-symmetric solution.

Lyon \& Leal (1998) performed experiments of concentrated suspension flow across the narrow gap of a rectangular channel. Their results were compared with the predictions of three analytical models. These models are: diffusive flux model, suspension balance model (McTigue \& Jenkins, 1992; Nott \& Brady, 1994), and a model developed by Mill \& Snabre (1995). In the experiments they measured, using a modified laser-Doppler velocimetry method, the fully developed particle velocity and concentration profiles, as well as the mean-square amplitudes of velocity fluctuations. The results were qualitatively consistent with the theoretical predictions from all the three models. Additionally, the Mills \& Snabre and suspension balance models were found to provide a better quantitative fit to the experimental data than the diffusive flux model.

Modifications of the original Phillips model have been made. Krishnan et al. (1996) proposed an additional migration flux originated from the flow curvature. Then Graham et al. (1998) proposed that the empirical parameter, related to shearinduced migration of the original Phillips model, to be a linear function of the particle volume fraction. Later Kim et al. (2008) performed a numerical and experimental comparison of these two models together with the original Phillips model in concentric Couette and parallel plate flow. They found that the accuracy of the three models in concentric Couette problem is comparable except that the Graham et al. model shows improved predictions near the inner cylinder. In the parallel plate problem the Krishnan et al. and Graham et al. models predict no inward migration, which is in good agreement with the experiments.

The diffusive flux model was also used by Yezaz \& Singh (2011) to carry out simulation of suspension flow in an asymmetric T-junction bifurcation channel. Using the finite volume method, they demonstrate a close agreement between the simulation and experimental results of Xi \& Shapley (2008). Their simulation showed that the concentration distributions are almost equally 
partitioned between the two downstream branches, even if the channel is asymmetric.

Although many industrial slot coating processes uses concentrated suspension of particles as the coating liquid, there are few studies about particle migration in slot coating geometry. Min \& Kim (2010) investigated the particle migration in free surface flows: planar jet flow and slot coating flow. They used the diffusive flux model (original Phillips et al. model) to predict the particle migration. The continuity, momentum and transport equations were solved using the commercial software Fluent ${ }^{\mathrm{TM}}$, based on the finite volume method. The result in the planar jet flow shows that, even though the velocity profile is fully developed and becomes flat, the particle distribution never reaches the uniform distribution. The slot coating system shows similar results on the distribution of particles at the downstream plane. The slot coating flow was analyzed just for a constant value of coated film thickness, greater than half of the coating gap. Another important observation of this work is that the particle concentration profile has a little change when the particles are convected to the downstream section; therefore the concentration profile in the coated film is practically the same as in the slot die. 


\section{2 \\ Mathematical Formulation of Free Surface Flow of Particle Suspension}

The flow of particle suspensions presents different characteristics from the flow of a Newtonian fluid with constant viscosity. This chapter presents the governing equations that describe flow of suspensions in a slot coating bead.

The suspension is considered a continuous medium, and the flow in the slot coating process bead is assumed to be laminar.

The flow is governed by the mass and linear momentum conservation equations. An additional equation is needed to trace the motion of solid particles in the fluid. In this work it will be used the convection-diffusion equation to describe the particle concentration distribution.

The slot coating flow presents two interfaces which positions are unknown a priori. Hence, extra equations and conditions that describe the position of these liquid-gas interfaces are also necessary to fully model the flow.

\section{1. Equation of Motion for Concentrated Suspensions}

Slot coating flow of concentrated suspension of solid particles as the coating liquid is the problem to be solved. A schematic representation of the problem is shown in Figure 2.1. The objective is to calculate the velocity, the pressure and the concentration fields in the flow domain. Additionally, this is a free surface problem, due to the presence of two interfaces (upstream and downstream), and therefore the position $(\mathrm{x}, \mathrm{y})$ of them must be calculated as part of the solution.

In order to have a better understanding of the problem, the boundary of the suspension flow in the slot coating process is divided into four (4) parts, as shown in Figure 2.1. 


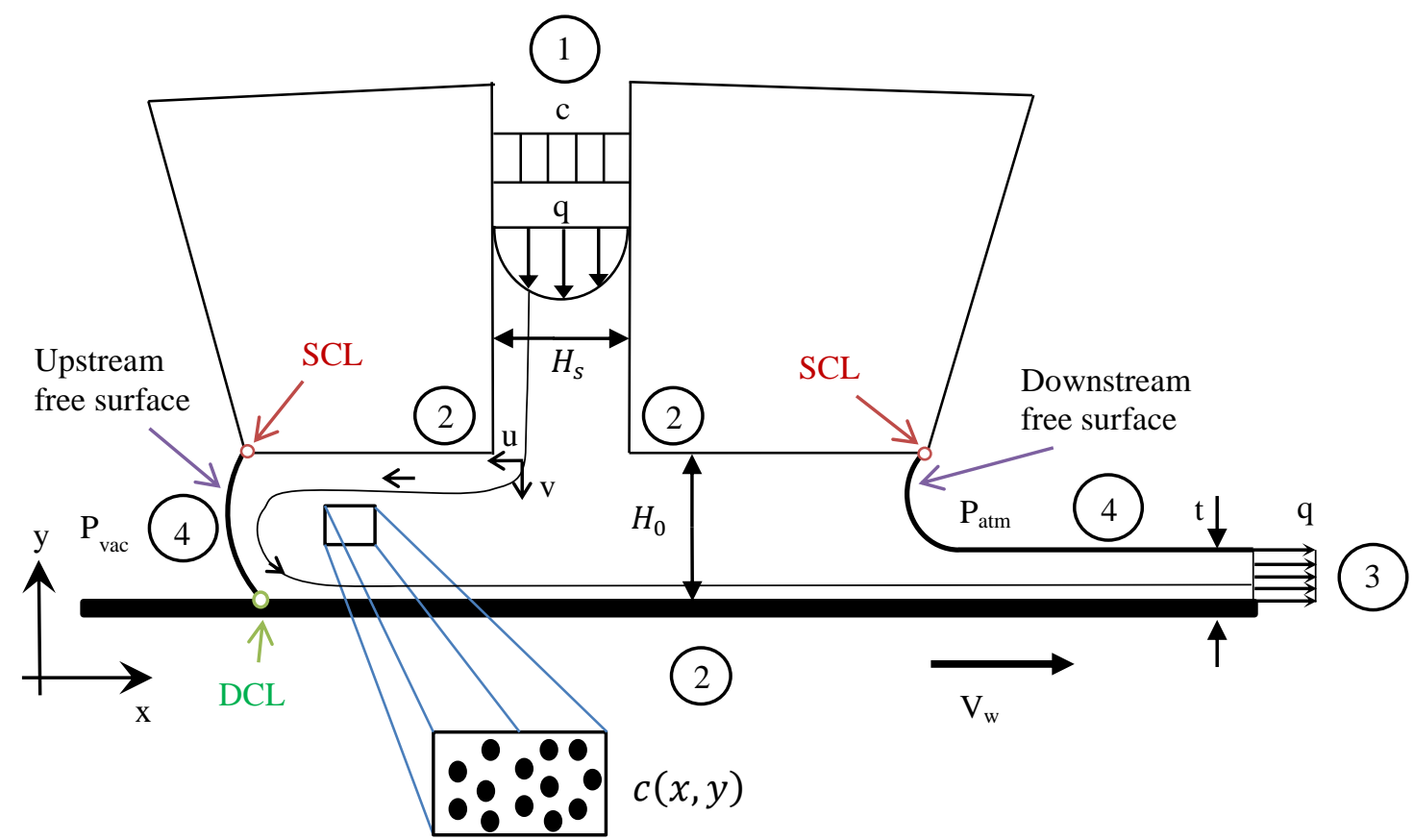

Concentration of particles per unit volume

Figure 2.1: Relevant variables involved in the slot coating process with suspension of particles.

The inflow velocity, at boundary 1 (see Figure 2.1), has a parabolic profile, while the concentration profile is taken to be constant. The particles migrate due to the different migration mechanisms, filling the whole domain at different concentrations, including the interfaces.

Along boundaries 2 (see Figure 2.1), the suspension is in contact with solid and rigid surfaces: upstream and downstream slot die surfaces, and substrate. The slot dies are still, while the substrate is moving at constant velocity.

At the outflow boundary 3 (see Figure 2.1), it is considered that the flow reaches a fully developed state. This boundary has to be placed at a sufficiently far away distance from the slot, for the approximation to be valid. Fully developed film over a substrate presents a solid body motion (constant velocity); therefore the shear-induced particle migration is zero.

The boundaries 4, shown in Figure 2.1, are the upstream and downstream interfaces.

There are six (6) unknowns or dependent variables for the problem:

- $u(x, y) \quad \rightarrow \quad$ Component of the velocity in the $\mathrm{x}$ direction.

- $v(x, y) \quad \rightarrow \quad$ Component of the velocity in the y direction. 
- $p(x, y) \quad \rightarrow \quad$ Pressure.

- $c(x, y) \quad \rightarrow \quad$ Concentration.

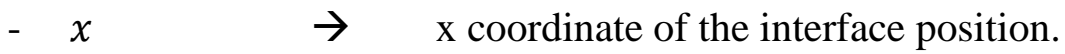

- $y \quad \rightarrow \quad$ y coordinate of the interface position.

While there are six (6) equations:

- One equation for mass conservation principle.

- Two equations for linear momentum conservation principle.

- One equation for particle conservation principle.

- Two equations for interface position.

The number of equations matches the number of unknowns; therefore it is possible to obtain a solution.

Additionally, Figure 2.1 shows the relevant variables and parameters involved in the flow of suspensions in slot coating process.

- $\quad q$ : Flow rate per unit width, $\left[\mathrm{m}^{2} / \mathrm{s}\right]$.

- $\quad t$ : Substrate thickness, $[m]$.

- $\quad V_{w}$ : Web velocity or substrate velocity, $[\mathrm{m} / \mathrm{s}]$.

- $\quad H_{0}$ : Coating gap, $[m]$.

- $\quad P_{v a c}:$ Vacuum pressure, $[P a]$.

- Patm: Atmospheric pressure, $[P a]$.

- $\quad c$ : Concentration of particles per unit volume, $\left[\mathrm{m}^{3} / \mathrm{m}^{3}\right]$.

- SCL: Dynamic contact line.

- DCL: Static contact line.

Several assumptions are made in the study of slot coating flow of particles suspensions. These hypotheses help us to simplify the equations of motion, but without losing accuracy.

i. Non-colloidal suspension.

ii. Steady state flow.

iii. Incompressible liquid phase.

iv. Neutrally buoyant, spherical and rigid particles.

v. Two-dimensional flow. 
vi. Inertial effects are negligible.

vii. Gravitational effects are negligible.

viii. Rigid slot dies and rigid substrate.

ix. No sources and/or sinks of particles in the suspension flow.

\subsection{1.}

\section{Governing Equations}

The equations derived from the principles of mass and linear momentum conservation (Panton, 2005) are presented. Here, the suspension (fluid particle and solid particles) is considered as a continuum medium; thus, the equations of mass conservation (continuity equation) and linear momentum conservation (momentum equation) are:

- Continuity equation:

$$
\frac{\partial \rho}{\partial t}+\nabla \cdot(\rho \underline{u})=0
$$

- Momentum equation:

$$
\frac{\partial}{\partial t}(\rho \underline{u})+\nabla \cdot(\rho \underline{u} \underline{u})=\rho \underline{g}+\nabla \cdot \underline{T}
$$

By assuming that both the liquid and solid phases are incompressible and have the same density, we can state that the density of the suspension is constant, therefore $\nabla \rho=0$.

Using the hypotheses the resulting continuity and momentum equations are:

$$
\begin{aligned}
& \nabla \cdot \underline{u}=0 \\
& \nabla \cdot \underline{T}=0
\end{aligned}
$$


The stress tensor, $\underline{T}$, can be decomposed in terms of the pressure, $p$, and viscous stress tensor, $\underline{\underline{\tau}}$ :

$$
\underline{\underline{T}}=-p \underline{\underline{I}}+\underline{\underline{\tau}}
$$

$\underline{I}$ is the identity tensor.

It has been shown that under special conditions the suspensions can be modeled as a Newtonian fluid. Then, the viscous stress tensor is given as:

$$
\underline{\underline{\tau}}=\eta(c) \underline{\underline{\gamma}}
$$

The viscosity, $\eta(c)$, depends on the volume fraction of particles. The empirical viscosity equation used here is the equation 1.9, with maximum packing volume fraction equal to 0.68 . Then, the equation for viscosity results:

$$
\eta_{r}=\left(1-\frac{c}{c_{m}}\right)^{-1.82}
$$

While the rate-of-strain tensor or shear rate, $\underline{\underline{\dot{\gamma}}}$, is given by:

$$
\underline{\underline{\gamma}}=\nabla \underline{u}+\nabla \underline{u}^{T}
$$

The conservation equations for the suspension flow were presented in equation 2.3 and equation 2.4. An additional equation is presented based on the mass conservation of solid particles in every infinitesimal volume of the suspension. The resulting equation is the convection-diffusion equation, which describes the spatiotemporal evolution of particle volume fraction, $c$, in the suspension flow. The equation that describes transport of specie in a flow is commonly given in the following form (Stocker, 2011): 


$$
\frac{\partial c}{\partial t}=-\nabla \cdot(\underline{u} c)+\nabla \cdot(D \nabla c)+R
$$

The term $-D \nabla c$ accounts for diffusive particle flux mechanism due to Brownian motion. The term $R$, accounts for sources and/or sinks operating inside the suspension.

A generalized steady state form of the equation 2.9, for dispersed phase conservation, that takes into account the different migration mechanisms and using hypothesis ix. and ii. is as follow:

$$
0=-\nabla \cdot(\underline{u} c)-\nabla \cdot\left(\underline{N_{t}}\right)
$$

where $\underline{N}_{t}$ is the total particles flux that accounts for different migration mechanisms such as sedimentation, Brownian diffusion, shear-induced, viscosity gradient-induced, or curvature-induced migration.

In this research, the action of Brownian diffusion and sedimentation are neglected, because of the hypotheses of non-colloidal suspension (hypothesis i.) and neutrally buoyant solid particles (hypothesis iv.), respectively. As a first approximation, the curvature-induced migration is neither taken into account. Neglecting Brownian diffusion implies that, as could be seen in equation 1.5, the Brownian forces are very small compared with the viscous forces; therefore the Péclet number is very large. This is one of the conditions that were presented in the introduction chapter (Figure 1.12) to model the suspension as a Newtonian fluid.

In this research it is considered the shear-induced and viscosity gradientinduced migration mechanisms. The concept and constitutive equations are presented in the next paragraph.

The model used in this research is the diffusive flux model developed originally by Phillips et al. (1992). It was chosen this model because of the simplicity in its implementation. The model studied in this research considers two migration mechanisms:

1. Effect of spatially varying interaction frequency (shear rate gradient induced migration): This migration mechanism occurs due to the 
frequency of collisions that the particles suffer. Experimentally it was observed that in a concentrated suspension the collision of particles are irreversible, resulting in net displacement of a particle from its original streamline. Particles collide between each other due to the difference in relative velocity of adjacent streamlines, i.e. due to shear rate gradient. The collision frequency is proportional to the shear rate gradient, resulting in higher collision frequencies between particles in regions with high shear rate gradient. Physically, in this series of collisions the particle migrate normal to its shearing surface, to adjacent streamlines with lower collision frequencies, as it is shown in Figure 2.2-a. The resulting constitutive equation for the particle flux due to the spatial variation in the interaction frequency is:

$$
\underline{N}_{c}=-k_{c} a^{2}\left(c^{2} \nabla \dot{\gamma}+c \dot{\gamma} \nabla c\right)
$$

where $a$ is the particle radius, and $k_{c}$ is a proportionality constant fitted from experimental data.

2. Effect of spatially varying viscosity (viscosity gradient induced migration): This migration mechanism occurs due to the resistance to motion of a rigid particle in regions with higher viscosity. In Figure 2.2b there is a flow with constant viscosity field, in which after collision, the two particles have the same displacement, because they experience the same resistance to motion. While in Figure 2.2-c, flow with viscosity gradient, during a collision the particle displacement is higher in the low viscous regions rather than in the high viscous ones; therefore, there is a net flux of particles in that direction. The resulting constitutive equation for the particle flux due to the spatial variation in the viscosity is:

$$
N_{\eta}=-k_{\eta} \dot{\gamma} c^{2}\left(\frac{a^{2}}{\eta}\right) \frac{d \eta}{d c} \nabla c
$$


where $k_{\eta}$ is a proportionality constant fitted from experimental data. It is noted that in the equation, the viscosity gradient is expressed in terms of concentration gradient, because of its direct proportional relationship observed of the viscosity model (equation 2.7 ).

In the model, it is considered that this two migration mechanisms are independent. Hence, the total flux of particles, $\underline{N}_{t}$, of the equation 2.10 has the form:

$$
\underline{N_{t}}=\underline{N}_{c}+\underline{N}_{\eta}
$$

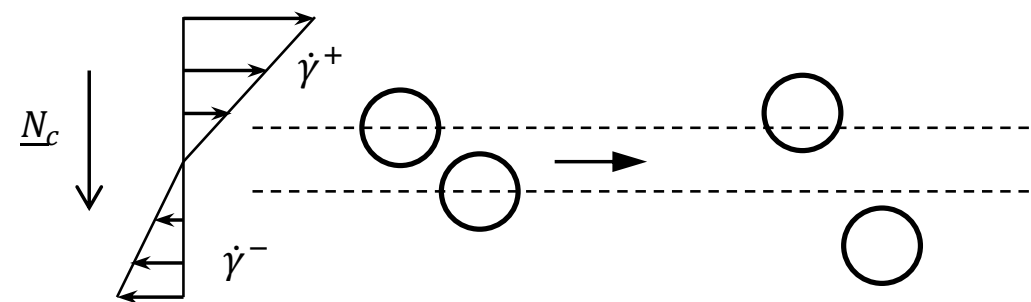

(a)

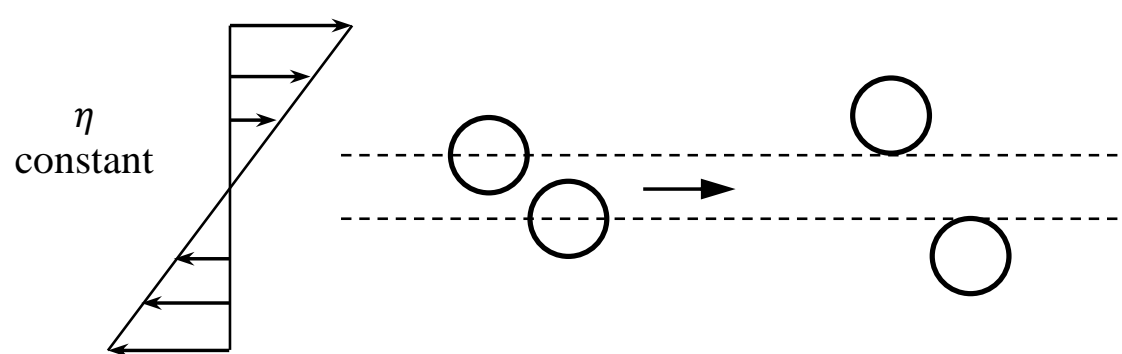

(b)

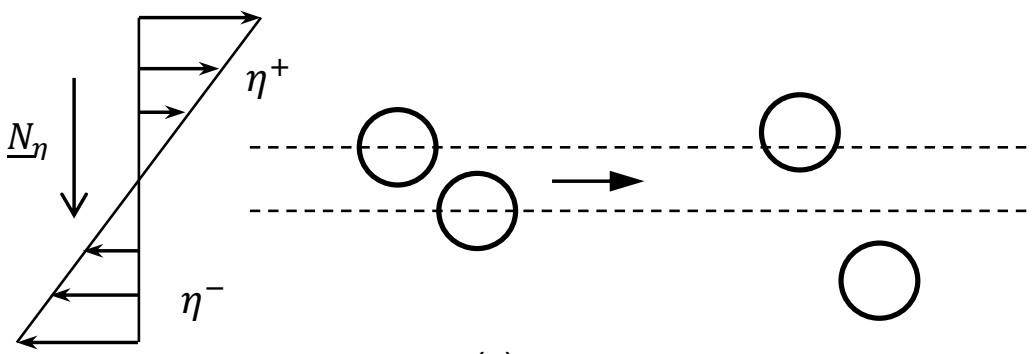

(c)

Figure 2.2: Irreversible, two body collisions with (a) spatially varying shear-rate, (b) constant viscosity and (c) spatially varying viscosity. 
Substituting equation 2.11, 2.12 and 2.13 into equation 2.10 yields the particle transport equation used in this work:

$$
0=-\underline{u} \cdot \nabla c-\nabla \cdot\left(-k_{c} a^{2}\left(c^{2} \nabla \dot{\gamma}+c \dot{\gamma} \nabla c\right)-k_{\eta} \dot{\gamma} c^{2}\left(\frac{a^{2}}{\eta}\right) \frac{d \eta}{d c} \nabla c\right)
$$

The resulting particle transport equation that takes into account the two particle migration mechanisms is developed and rearranged into its final form as:

$$
\underline{u} \cdot \nabla c=\nabla \cdot(\bar{D} \nabla c)+k_{c} a^{2} \nabla \cdot\left(c^{2} \nabla \dot{\gamma}\right)
$$

The term $\bar{D}$, works like a modified diffusion coefficient. It was rearranged in this form to get a separated expression for particle migration due to gradient in particle concentration, and gradient in shear rate. The expression for the term $\bar{D}$ is as follows:

$$
\bar{D}=k_{c} a^{2} c \dot{\gamma}+k_{\eta} \dot{\gamma} c^{2} \frac{a^{2}}{\eta} \frac{d \eta}{d c}
$$

The final governing equations are:

- Continuity equation: equation 2.3.

- Momentum equation: equation 2.4.

- Transport equation: equation 2.15.

We also need a set of equations to calculate the coordinates and consequently, the free surface positions. It will be presented in the discussion the mesh elliptic generation technique and numerical mapping here implemented.

\subsection{2.}

\section{Boundary Conditions for the Free Surface Problem}

This section presents the boundary conditions necessary to solve the slot coating flow with a concentrated suspension. These conditions are applied to the 
governing equations (equation 2.3, equation 2.4 and equation 2.15), i.e. for the concentrated suspension flow and for the particle flux.

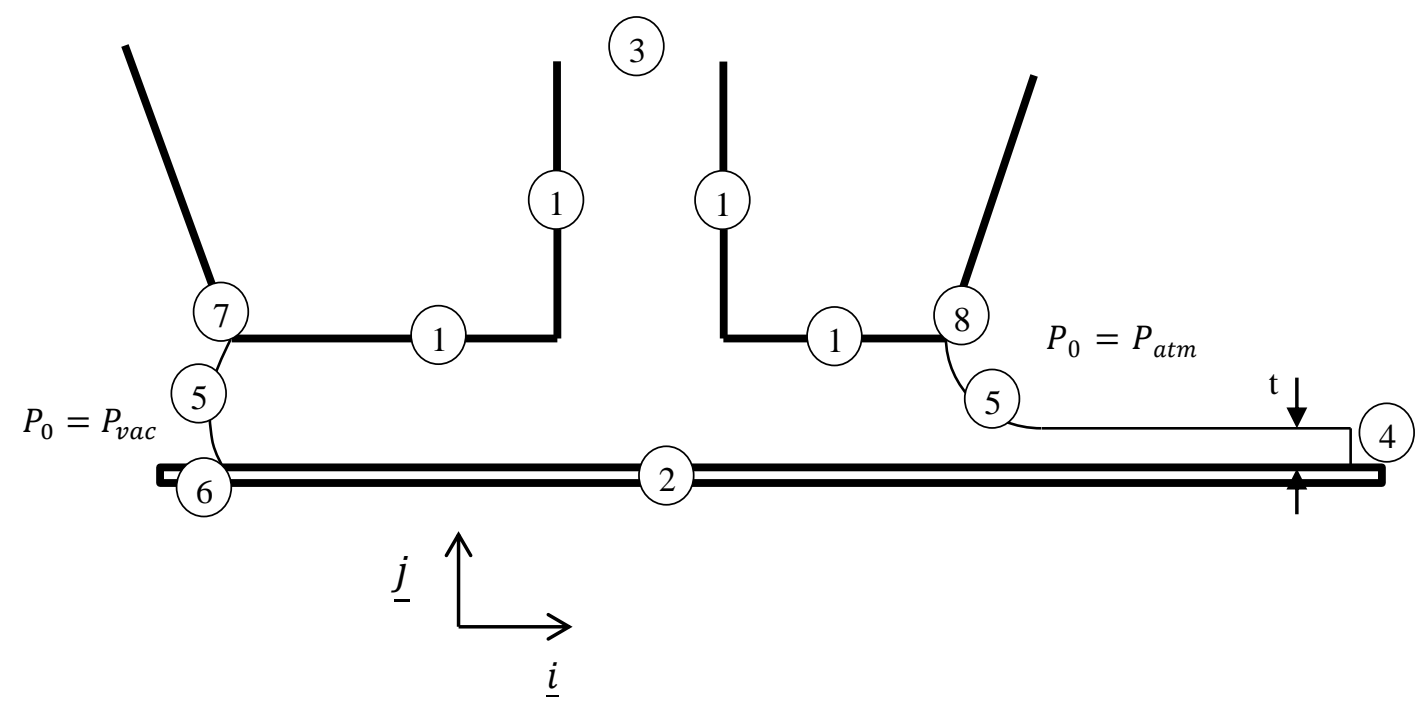

Figure 2.3: Scheme of the division of the boundaries.

Figure 2.3 labels the different boundaries of the flow domain. The boundary conditions for the momentum (equation 2.4) equation are:

1. Boundary 1: No-slip and no-penetration. The suspension has the same velocity of the slot die walls.

$$
\underline{u}=0
$$

2. Boundary 2: No-slip and no-penetration. The suspension has the same velocity of the substrate.

$$
\underline{u}=V_{w} \underline{i}
$$

3. Boundary 3: Prescribed velocity profile for the suspension at the inlet of the feed slot.

$$
\underline{u}=-\frac{6 q}{H_{s}} *\left[\left(\frac{x}{H_{s}}\right)-\left(\frac{x}{H_{s}}\right)^{2}\right] \underline{j}
$$


4. Boundary 4: Developed flow and fixed pressure. The suspension flow at this boundary is assumed to be fully developed, i.e. the velocity profile remains unchanged in the $\mathrm{x}$-direction. Moreover, the pressure is set to atmospheric pressure.

$$
\begin{aligned}
& \underline{n} \cdot \nabla \underline{u}=0 \\
& P=P_{a t m}
\end{aligned}
$$

$\underline{n}$ is an outward unit vector normal to the boundary surface $(\underline{n}=\underline{i})$.

5. Boundary 5: Interface. This boundary is the interface between the suspension and the surrounding gas. There are two conditions applied here: force balance and kinematic condition (no flux through the interface).

The stress balance at the interface can be decomposed in two directions: tangential and normal stress. Because of the low viscosity of the gas (air) compared with the one of the suspension, the tangential stress of the gas is neglected. While in the normal direction there is balance between the suspension stress, the capillary pressure and the gas pressure.

$$
\underline{n}_{f} \cdot \underline{T}=\sigma \frac{d \underline{t} f}{d s}-\underline{n}_{f} \cdot P_{0}
$$

$\underline{t}_{f}$ and $\underline{n}_{f}$ are the unit vector tangent and normal to the free surface, respectively, $\sigma$ is the surface tension of the suspension, and $P_{0}$ is the ambient pressure. At the upstream interface $P_{0}$ equals the vacuum pressure, $P_{v a c}$; while at the downstream interface it takes the value of the atmospheric pressure, $P_{a t m}$. 
The other condition is that there is no mass flow through the interface.

$$
\underline{n}_{f} \cdot \underline{u}=0
$$

6. Corner 6: Dynamic contact line (DCL). It is applied the Navier slip condition.

$$
\frac{1}{\beta} \underline{t}_{w} \cdot\left(\underline{v}-\underline{V}_{w}\right)=\underline{t}_{w} \cdot(\underline{T} \cdot \underline{n})
$$

$\beta$ is the slip coefficient, $\underline{t}_{w}$ is the unit vector tangential to the substrate. In addition, a constant dynamic contact angle, $\theta_{d}$, is set between the substrate and the interface.

$$
\underline{n}_{w} \cdot \underline{n}_{f}=\cos \left(\theta_{d}\right)
$$

$\underline{n}_{w}$ is the unit vector normal to the solid wall (substrate) and directed into the liquid.

7. Corner 7: Upstream static contact line (USCL). This line is free to move over the upstream die lip, therefore it has always the same ycoordinate.

$$
\underline{j} \cdot \underline{x}=H_{0}
$$

The static contact angle, $\theta_{s}$, is the angle between the normal to the slot wall (directed into the liquid) and the normal to the free surface at the contact line. This angle is specified.

$$
\underline{n}_{w} \cdot \underline{n}_{f}=\cos \left(\theta_{s}\right)
$$


8. Corner 8: Downstream static contact line (DSCL). This line is fixed at the edge of the downstream die lip. Hence, both the $\mathrm{x}$ and $\mathrm{y}$ coordinate are constant.

$$
\underline{x}_{s d}=\underline{x}_{e d g e}
$$

The boundary conditions for the transport equation (equation 2.15) are:

1. Boundaries 1,2 and 5: There is no-flux of particles at solid and free surfaces boundaries.

$$
\underline{n} \cdot\left(\underline{N_{c}}+\underline{N_{\eta}}\right)=0
$$

2. Boundary 3: Prescribed concentration profile. It is set a constant value for the concentration, $c_{b u l k}$.

$$
c(x, y)=c_{b u l k}
$$

3. Boundary 4: Developed flow and concentration profile. The concentration gradient in the $\mathrm{x}$-direction is set zero; therefore, there is no flux of particles due to the migration mechanisms considered.

$$
\underline{n} \cdot \nabla c=0
$$

There is still an issue to be solved, the flow domain is unknown and is part of the solution. The governing equations and boundary conditions are defined in an unknown domain. In the following section, we will present the method used to find the free surface location simultaneously with velocity, pressure and particle concentration field. 


\section{2.}

\section{Solution of Free Surface Problems}

In free surface problems, the flow domain, where the governing equations and boundary conditions that describe the phenomenon are defined, is not known a priori and must be calculated as part of the solution. This is the case here, because of the presence of both the upstream and downstream air-liquid interfaces. In this section, it is presented a methodology to incorporate the freesurface location as new unknowns to the problem, which allows finding them simultaneously with the other variables.

The domain of the problem, which is called the physical domain $\Omega$, is unknown; therefore, the governing equations and boundary conditions that describe the flow have to be transformed to an equivalent system defined in a known fixed domain, which is called fixed reference domain $\bar{\Omega}$. This transformation is made through a mapping, which relates both domains.

The mapping $\underline{x}=\underline{x}(\underline{\xi})$, called direct mapping, is a function that relates both domains $(\bar{\Omega} \rightarrow \Omega)$. The physical domain is parameterized by the position vector $\underline{x}=(x, y)$, and the fixed reference domain is parameterized by $\underline{\xi}=(\xi, \eta)$, called inverse mapping. This transformation is achieved through the Jacobian matrix of the change of coordinates $\left(\nabla_{\xi} \underline{x}\right)$.

$$
\nabla_{\xi} \underline{x}=\frac{\partial \underline{x}}{\partial \underline{\xi}}=\boldsymbol{J}_{T}=\left(\begin{array}{ll}
\frac{\partial x}{\partial \xi} & \frac{\partial y}{\partial \xi} \\
\frac{\partial x}{\partial \eta} & \frac{\partial y}{\partial \eta}
\end{array}\right)
$$

By using this transformation, for a general function defined in the reference domain, $\phi=\phi(\xi, \eta)$, using the chain rule, their derivatives can be expressed in function in $\mathrm{x}$ and $\mathrm{y}$ coordinates of the physical domain. The mathematical relations are:

$$
\left(\begin{array}{l}
\frac{\partial \phi}{\partial \xi} \\
\frac{\partial \phi}{\partial \eta}
\end{array}\right)=J_{T}\left(\begin{array}{l}
\frac{\partial \phi}{\partial x} \\
\frac{\partial \phi}{\partial y}
\end{array}\right)
$$




$$
\begin{aligned}
& \frac{\partial \phi}{\partial x}=\frac{1}{\left|J_{T}\right|}\left(\frac{\partial y}{\partial \eta} \frac{\partial \phi}{\partial \xi}-\frac{\partial y}{\partial \xi} \frac{\partial \phi}{\partial \eta}\right) \\
& \frac{\partial \phi}{\partial y}=\frac{1}{\left|J_{T}\right|}\left(-\frac{\partial x}{\partial \eta} \frac{\partial \phi}{\partial \xi}+\frac{\partial x}{\partial \xi} \frac{\partial \phi}{\partial \eta}\right)
\end{aligned}
$$

The jacobian determinant, $J_{T}$, represents the relation between the differential areas of the two domains.

$$
\begin{gathered}
\left|\boldsymbol{J}_{T}\right|=J_{T}=\operatorname{det} \boldsymbol{J}_{T}=\frac{\partial x}{\partial \xi} \frac{\partial y}{\partial \eta}-\frac{\partial x}{\partial \eta} \frac{\partial y}{\partial \xi} \\
d \Omega=J_{T} d \bar{\Omega}
\end{gathered}
$$

This mapping has to satisfy two conditions:

1. The boundary of the reference domain has to be mapped over the boundary of the physical domain.

$$
\bar{\Gamma}=\Gamma
$$

2. The mapping has to be invertible.

$$
\left|\boldsymbol{J}_{T}\right|>0
$$

It is common to name the equations that govern the mapping as the mesh generation equations. Part of the solution is to calculate the mapping function. There are different types of mapping methods. In the present work it is used the Elliptic Mesh Generation Method (Benjamin, 1994, Christodoulou \& Scriven, 1992). 


\subsection{1.}

\section{Elliptic Mesh Generation}

In the case of slot coating flow the mapping, represented in Figure 2.4, is not known due to the two interface positions. The equation that describes the mapping between the physical and reference domain needs to be defined. The Elliptic Mesh Generation method, used in this research, was proposed by Benjamin (1994), which is a generalization of the method developed by Christodoulou \& Scriven (1992).

In this method, the inverse mapping has to satisfy the following elliptic differential equation.

$$
\begin{aligned}
& \nabla \cdot\left(D_{\xi} \nabla \xi\right)=0 \\
& \nabla \cdot\left(D_{\eta} \nabla \eta\right)=0
\end{aligned}
$$

$D_{\xi}$ and $D_{\eta}$ are the diffusion coefficients of the coordinates $\xi$ and $\eta$, and they control the spacing between the level curves (constant values) for $\xi$ and $\eta$. Equations 2.40 and 2.41 are like the equations of conductive heat transfer with variable thermal conductivity.

The general form of these equations could be written as:

$$
\nabla \cdot \underline{D} \cdot \nabla \underline{\xi}=0
$$

$\underline{D}$ is the diffusion coefficients tensor.

These equations, defined in the physical domain, describe the inverse mapping. Then, these equations are rewritten in the reference domain, obtaining in this way the coordinates of the physical domain in function of the coordinates of the reference domain.

The simplest reference domain to be used is a quadrangular one, divided in unitary squares. In Figure 2.4 could be observed the physical domain divided in six quadrangular regions, each of them mapped in six reference squares. 
Boundary conditions are needed to solve the two partial differential equations presented.

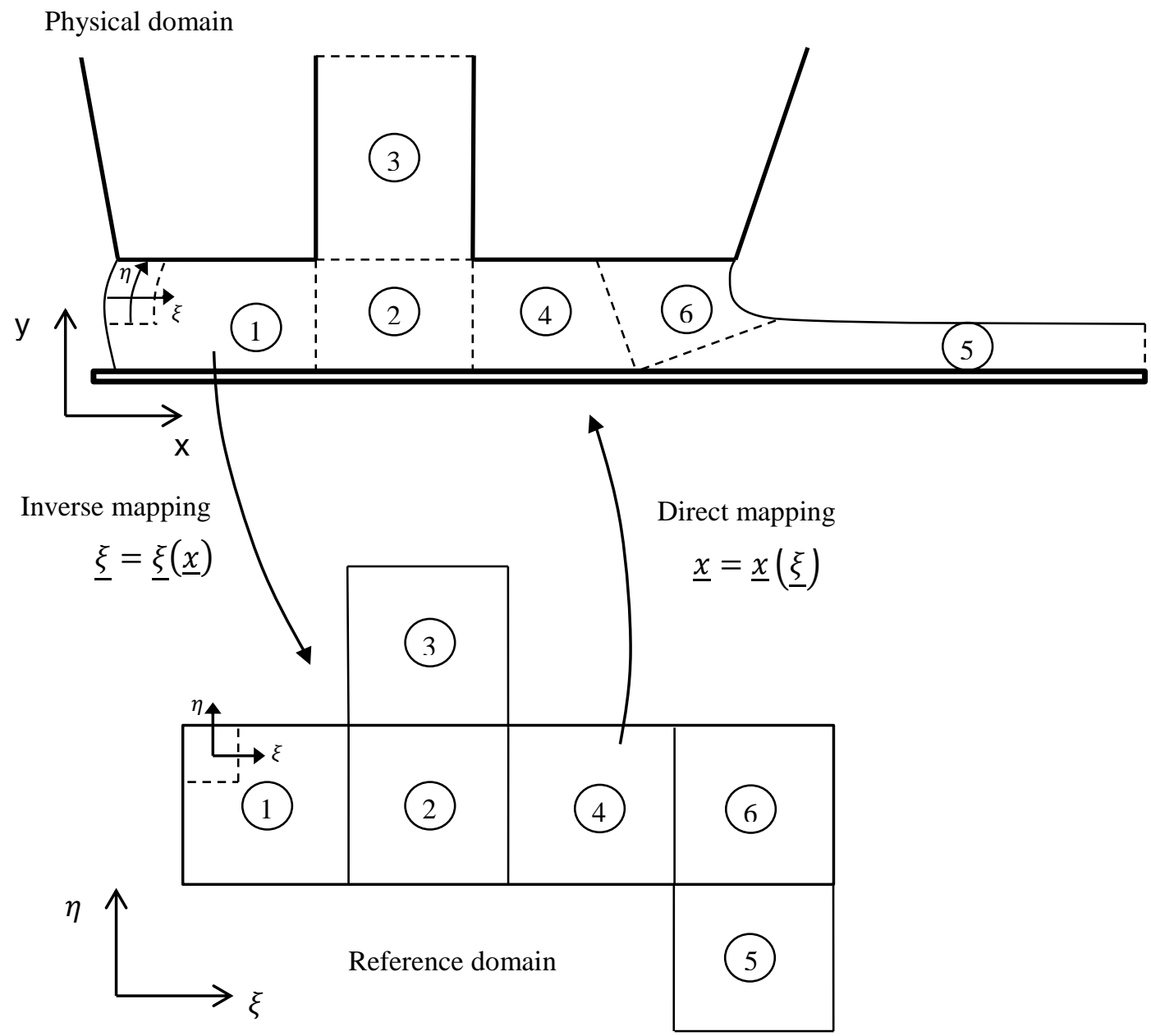

Figure 2.4: Scheme of the mapping.

\subsection{2.}

\section{Boundary Conditions of Mesh Equations}

There are many types of boundary conditions for the mesh equations. Some examples are:

1. Prescribed angle: the angle, $\theta$, between the coordinates $\xi$ and $\eta$ is prescribed over the boundary.

$$
\underline{n} \cdot \nabla \xi=|\nabla \xi| \cos \theta
$$


2. Slide over a boundary side: The nodes are free to slide over the boundary at a line which equation is known.

$$
f(\underline{x})=0
$$

3. Fixed nodes: The nodal position is fixed.

4. Stretching function: A distribution function is used to distribute the nodes over the boundary.

$$
\xi=f^{-1}(s), \quad \eta=g^{-1}(s)
$$

5. Kinematic condition: The fluid cannot flow through the free surface. This boundary condition is the kinematic condition that couples the flow and the mesh generation equation.

$$
\underline{n} \cdot \underline{u}=0
$$

This equation locates implicitly the position of a free surface. 


\section{3 \\ Solution Method for the System of Differential Equations}

The set of partial differential equations that governs the free surface flow of a suspension of particles in the slot coating process is solved using the Galerkin Finite Element Method (GFEM).

\section{1. \\ Solution Method by the GFEM}

The governing equations are expressed in their weak or variational form in order to solve them by the Galerkin method.

Before presenting the weak form, the original set of differential equations is augmented to enable the use of the usual finite element representation of the unknown velocity field. It is created a new variable that account for velocity field first derivatives.

In the constitutive equation for particle migration due to shear-rate gradient (equation 2.11), the term $\nabla \dot{\gamma}$ must be evaluated. This term is a function of the second derivatives of the velocity as shown below. In the Cartesian coordinate system, the deformation rate gradient is expressed as:

$$
\nabla \dot{\gamma}=\frac{\partial \dot{\gamma}}{\partial x} \underline{i}+\frac{\partial \dot{\gamma}}{\partial y} \underline{j}
$$

The deformation rate, $\dot{\gamma}$, is defined (Bird et al., 1977) as:

$$
\dot{\gamma}=\sqrt{\frac{1}{2} I I}
$$

where $I I$ is the second invariant of the shear rate tensor, defined as the trace of the square of this tensor: 


$$
I I=\operatorname{tr} \underline{\underline{\underline{\gamma}}}^{2}
$$

Combining equations 2.8, 3.2 and 3.3, an expression for the deformation rate in the Cartesian coordinate is derived:

$$
\dot{\gamma}=\left[2\left(\frac{\partial u}{\partial x}\right)^{2}+2\left(\frac{\partial v}{\partial y}\right)^{2}+\left(\frac{\partial u}{\partial y}+\frac{\partial v}{\partial x}\right)^{2}\right]^{0.5}
$$

The components of the equation 3.1 are:

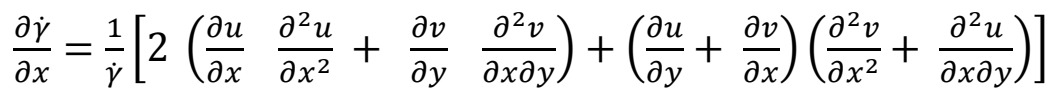

$$
\begin{aligned}
& \frac{\partial \dot{\gamma}}{\partial y}=\frac{1}{\dot{\gamma}}\left[2\left(\frac{\partial u}{\partial x} \frac{\partial^{2} u}{\partial y \partial x}+\frac{\partial v}{\partial y} \frac{\partial^{2} v}{\partial y^{2}}\right)+\left(\frac{\partial u}{\partial y}+\frac{\partial v}{\partial x}\right)\left(\frac{\partial^{2} u}{\partial y^{2}}+\frac{\partial^{2} v}{\partial y \partial x}\right)\right]
\end{aligned}
$$

In the GFEM, the velocity field is usually written as a linear combination of continuous piece-wise polynomials. Therefore, along element boundaries, the velocity is continuous, but the velocity gradients are not. Therefore, the deformation rate gradient cannot be evaluated in this form. A common approach to avoid this problem is to represent the velocity gradient as a separate independent field that is also represented as a linear combination of continuous piece-wise polynomials. Because of this, an additional variable, $\underline{\underline{G}}$, is introduced to represent the velocity gradient with a continuous interpolation between the elements:

$$
\underline{\underline{G}}=\nabla \underline{u}
$$

The variable $\underline{\underline{G}}$ is called from now on interpolated velocity gradient.

In incompressible flows, as the one considered here, the trace of the velocity gradient vanishes, $\operatorname{tr} \underline{\underline{G}}=\nabla \cdot \underline{u}=0$. The approximate velocity field computed with the GFEM (described in the next section) does not satisfy this condition in every 
element, but it does in the whole domain. If the weighting functions used in the continuity equation (equation 2.3) and equation 3.7 are different, the trace of the interpolated velocity gradient becomes dangerously large in flow regions where the velocity changes abruptly. As suggested by Pasquali \& Scriven (2002), the incompressibility condition is guaranteed when $\underline{\underline{G}}$ is defined through a modified version of equation 3.7 :

$$
\underline{\underline{G}}=\nabla \underline{u}-\frac{\nabla \cdot \underline{u}}{\operatorname{tr} \underline{\underline{I}}} \underline{I}
$$

With this approach, the original system of PDEs is augmented by equation 3.8 .

The system of coupled PDEs is:

$$
\begin{gathered}
\nabla \cdot \underline{u}=0 \\
\nabla \cdot \underline{T}=0 \\
\underline{u} \cdot \nabla c=\nabla \cdot(\bar{D} \nabla c)+k_{c} a^{2} \nabla \cdot\left(c^{2} \nabla \dot{\gamma}\right) \\
\nabla \cdot \underline{D} \cdot \nabla \underline{\xi}=0 \\
\underline{\underline{G}}=\nabla \underline{u}-\frac{\nabla \cdot \underline{u}}{t r \underline{\underline{I}}} \underline{I}
\end{gathered}
$$

The stress tensor is defined (combining equations 2.5, 2.6, 2.7 and 2.8) as:

$$
\underline{T}=-p \underline{I}+\left(1-\frac{c}{c_{m}}\right)^{-1.82}\left(\nabla \underline{u}+\nabla \underline{u}^{T}\right)
$$


These equations are the continuity (equation 3.9), momentum (equation 3.10 ), transport (equation 3.11), mesh (equation 3.12), and interpolated velocity gradient (equation 3.13) equations respectively.

The weak form is written in the reference domain as:

$$
\begin{gathered}
R_{c}=\int_{\bar{\Omega}}(\nabla \cdot \underline{u}) \chi J_{T} d \bar{\Omega} \\
R_{\boldsymbol{m}}=\int_{\bar{\Omega}}(\underline{T}: \nabla \underline{W}) J_{T} d \bar{\Omega}-\int_{\bar{\Gamma}}(\underline{n} \cdot \underline{T}) \cdot \underline{W}\left(\frac{d \Gamma}{d \bar{\Gamma}}\right) d \bar{\Gamma}, \\
R_{\boldsymbol{t}}=\int_{\bar{\Omega}}\left[(\underline{u} \cdot \nabla c) \phi+\bar{D} \nabla c \cdot \nabla \phi+k_{c} a^{2} c^{2} \nabla \dot{\gamma} \cdot \nabla \phi\right] J_{T} d \bar{\Omega}- \\
\int_{\bar{\Gamma}}\left[\underline{n} \cdot\left(\bar{D} \nabla c+k_{c} a^{2} c^{2} \nabla \nabla \dot{\gamma}\right)\right] \phi\left(\frac{d \Gamma}{d \bar{\Gamma}}\right) d \bar{\Gamma}, \\
R_{x}=\int_{\bar{\Omega}}(\nabla \underline{W} \cdot \underline{D} \cdot \nabla \underline{\xi}) J_{T} d \bar{\Omega}-\int_{\bar{\Gamma}}(\underline{n} \cdot \underline{D} \cdot \nabla \underline{\xi}) \cdot \underline{W}\left(\frac{d \Gamma}{d \bar{\Gamma}}\right) d \bar{\Gamma}, \\
R_{G}=\int_{\bar{\Omega}}\left(\underline{\underline{G}}-\nabla \underline{u}+\frac{\nabla \cdot \underline{u}}{t r \underline{I}} \underline{\underline{I}}\right) \cdot \underline{\psi} J_{T} d \bar{\Omega},
\end{gathered}
$$

$\underline{W}=\left(\phi_{1}, \phi_{2}\right)$ is the weighting function vector for the momentum equation and mesh equation. $\phi$ is the weighting function for the particle transport equation. $\chi$ is the weighting function for the continuity equation. $\underline{\psi}=\left(\psi_{1}, \psi_{2}, \psi_{3}, \psi_{4}\right)$ is the weighting function vector for the interpolated velocity gradient equation.

The system is discretized by using a finite number of weighting functions and corresponding basis functions used to express the unknown fields.

The weighted residuals of each equation are written in Cartesian coordinates as:

- Weighted residual of the continuity equation:

$$
R_{c}^{i}=\int_{\bar{\Omega}}\left(\frac{\partial u}{\partial x}+\frac{\partial v}{\partial y}\right) \chi_{i} J_{T} d \bar{\Omega}
$$


- Weighted residual of the momentum equation:

$$
\begin{aligned}
& R_{m x}^{i}=\int_{\bar{\Omega}}\left(\frac{\partial \phi_{i}}{\partial x} T_{x x}+\frac{\partial \phi_{i}}{\partial y} T_{x y}\right) J_{T} d \bar{\Omega}-\int_{\bar{\Gamma}}(\underline{n} \cdot \underline{T})_{x} \phi_{i}\left(\frac{d \Gamma}{d \bar{\Gamma}}\right) d \bar{\Gamma} \\
& R_{m y}^{i}=\int_{\bar{\Omega}}\left(\frac{\partial \phi_{i}}{\partial x} T_{x y}+\frac{\partial \phi_{i}}{\partial y} T_{y y}\right) J_{T} d \bar{\Omega}-\int_{\bar{\Gamma}}(\underline{n} \cdot \underline{\underline{T}})_{y} \phi_{i}\left(\frac{d \Gamma}{d \bar{\Gamma}}\right) d \bar{\Gamma}
\end{aligned}
$$

- Weighted residual of the transport equation:

$$
\begin{aligned}
R_{t}^{i}=\int_{\bar{\Omega}}\left[\begin{array}{c}
\left(u \frac{\partial c}{\partial x}+v \frac{\partial c}{\partial y}\right)+\bar{D}\left(\frac{\partial \phi_{i}}{\partial x} \frac{\partial c}{\partial x}+\frac{\partial \phi_{i}}{\partial y} \frac{\partial c}{\partial y}\right)+ \\
+k_{c} a^{2} c^{2}\left(\frac{\partial \phi_{i}}{\partial x} \frac{\partial \dot{\gamma}}{\partial x}+\frac{\partial \phi_{i}}{\partial y} \frac{\partial \dot{\gamma}}{\partial y}\right)
\end{array}\right] \phi_{i} J_{T} d \bar{\Omega}- \\
\quad-\int_{\bar{\Gamma}}\left[\underline{n} \cdot\left(\bar{D} \nabla c+k_{c} a^{2} c^{2} \nabla \dot{\gamma}\right)\right] \phi_{i}\left(\frac{d \Gamma}{d \bar{\Gamma}}\right) d \bar{\Gamma}
\end{aligned}
$$

- Weighted residual of the mesh equation:

$$
\begin{aligned}
R_{x}^{i}= & -\int_{\bar{\Omega}} D_{\xi}\left(\frac{\partial y}{\partial \eta} \frac{\partial \phi_{i}}{\partial x}-\frac{\partial x}{\partial \eta} \frac{\partial \phi_{i}}{\partial y}\right) d \bar{\Omega}+ \\
& +\int_{\bar{\Gamma}} D_{\xi} \frac{1}{J_{T}}\left(\frac{\partial y}{\partial \eta} n_{x}-\frac{\partial x}{\partial \eta} n_{y}\right) \phi_{i}\left(\frac{d \Gamma}{d \bar{\Gamma}}\right) d \bar{\Gamma} \\
R_{y}^{i}= & -\int_{\frac{\Omega}{\Omega}} D_{\eta}\left(\frac{\partial y}{\partial \xi} \frac{\partial \phi_{i}}{\partial x}-\frac{\partial x}{\partial \xi} \frac{\partial \phi_{i}}{\partial y}\right) d \bar{\Omega} \\
& +\int_{\bar{\Gamma}} D_{\eta} \frac{1}{J_{T}}\left(-\frac{\partial y}{\partial \xi} n_{x}-\frac{\partial x}{\partial \xi} n_{y}\right) \phi_{i}\left(\frac{d \Gamma}{d \bar{\Gamma}}\right) d \bar{\Gamma}
\end{aligned}
$$

- Weighted residual of the interpolated velocity gradient equation:

$$
R_{u x}^{i}=\int_{\bar{\Omega}}\left[G_{u x}-\frac{\partial u}{\partial x}+\frac{1}{2}\left(\frac{\partial u}{\partial x}+\frac{\partial v}{\partial y}\right)\right] \psi_{i} J_{T} d \bar{\Omega}
$$




$$
\begin{array}{r}
R_{u y}^{i}=\int_{\bar{\Omega}}\left[G_{u y}-\frac{\partial u}{\partial y}\right] \psi_{i} J_{T} d \bar{\Omega} \\
R_{v x}^{i}=\int_{\bar{\Omega}}\left[G_{v x}-\frac{\partial v}{\partial x}\right] \psi_{i} J_{T} d \bar{\Omega} \\
R_{v y}^{i}=\int_{\bar{\Omega}}\left[G_{v y}-\frac{\partial v}{\partial y}+\frac{1}{2}\left(\frac{\partial u}{\partial x}+\frac{\partial v}{\partial y}\right)\right] \psi_{i} J_{T} d \bar{\Omega}
\end{array}
$$

The unknowns are now represented as a linear combination of basis functions, which, in GFEM, are the same as the corresponding weighting functions.

The unknown fields (independent variables), velocity $u$ and $v$, pressure $p$, particle concentration $c$, coordinates of the mesh nodes $x$ and $y$, and interpolated velocity gradient $G_{u x}, G_{u y}, G_{v x}$ and $G_{v y}$ are written as:

$$
\begin{gathered}
u=\sum_{j=1}^{n}\left(U_{j} \phi_{j}\right) \quad, \quad v=\sum_{j=1}^{n}\left(V_{j} \phi_{j}\right) \\
p=\sum_{j=1}^{m}\left(P_{j} \chi_{j}\right) \\
c=\sum_{j=1}^{n}\left(c_{j} \phi_{j}\right) \\
x=\sum_{j=1}^{n}\left(X_{j} \phi_{j}\right), \quad y=\sum_{j=1}^{n}\left(Y_{j} \phi_{j}\right) \\
G_{u x}=\sum_{j=1}^{l}\left(G u x_{j} \psi_{j}\right) \quad, \quad G_{u y}=\sum_{j=1}^{l}\left(G u y_{j} \psi_{j}\right) \\
G_{v x}=\sum_{j=1}^{l}\left(G v x_{j} \psi_{j}\right) \quad, \quad G_{v y}=\sum_{j=1}^{l}\left(G v y_{j} \psi_{j}\right)
\end{gathered}
$$

The basis functions used to represent the independent variables inside each element are: $\phi_{j}(\xi, \eta)$ Lagrangian biquadratic polynomials, $\chi_{j}(\xi, \eta)$ linear discontinuous, and $\psi_{j}(\xi, \eta)$ Lagrangian bilinear polynomials. 
$U_{j}, V_{j}, P_{j}, c_{j}, X_{j}, Y_{j}, G u x_{j}, G u y_{j}, G v x_{j}$, and $G v y_{j}$ are the coefficients of the linear combination of basis functions. They represent the unknowns of the discrete problem.

The basis functions described before are associated with a quadrilateral element with 9 nodes and 64 degrees of freedoms. Inside each element, the local degrees of freedoms are numbered as shown in Table 3.1.

Table 3.1: Degrees of freedom at each element.

\begin{tabular}{|c|c|}
\hline Variables & Local numbering \\
\hline $\mathrm{y}$ & $\mathbf{1 - 9}$ \\
\hline $\mathrm{x}$ & $\mathbf{1 0 - 1 8}$ \\
\hline $\mathrm{v}$ & $\mathbf{1 9 - 2 7}$ \\
\hline $\mathrm{u}$ & $\mathbf{2 8 - 3 6}$ \\
\hline $\mathrm{c}$ & $\mathbf{3 7 - 4 5}$ \\
\hline $\mathrm{p}$ & $\mathbf{4 6 - 4 8}$ \\
\hline Gux & $\mathbf{4 9 - 5 2}$ \\
\hline Guy & $\mathbf{5 3 - 5 6}$ \\
\hline Gvx & $\mathbf{5 7 - 6 0}$ \\
\hline Gvy & $\mathbf{6 1 - 6 4}$ \\
\hline
\end{tabular}

The choice of the basis functions is not arbitrary, because the convergence of the method could be affected. As shown by Sani et al. (1981), in the solution of fluid flow problem with Newtonian fluids, the combination of biquadratic interpolating function for velocity and discontinuous linear ones for the pressure, gives a better numerical performance that other alternatives.

\section{2. \\ Solution of the Nonlinear System of Algebraic Equations by Newton's Method}

The integration of the weighted residual equations is performed numerically, using the Gaussian Quadrature Method with three points of integration in each direction.

When the independent variables are written in terms of the linear combination of basis functions, the system of partial differential equations is transformed into a system of nonlinear algebraic equations. This system can be expressed in a compact notation: 


$$
\underline{\boldsymbol{R}}(\underline{\boldsymbol{c}} ; \underline{\boldsymbol{p}})=\mathbf{0},
$$

where $\boldsymbol{R}$ is the vector of weighted residuals. $\underline{\boldsymbol{c}}$ represents the solution vector, it contains the basis function coefficients that are the unknowns of the problem. Finally, $\underline{\boldsymbol{p}}$ is the vector of parameters that control the problem. Equation 3.35 is solved using the Newton's iterative method, that follows the iterative procedure shown below:

$$
\begin{aligned}
& \underline{\boldsymbol{J}} \delta \underline{\boldsymbol{c}}=-\underline{\boldsymbol{R}}(\underline{\boldsymbol{c}} ; \underline{\boldsymbol{p}}) \\
& \underline{\boldsymbol{c}}^{(k+1)}=\underline{\boldsymbol{c}}^{(k)}+\delta \underline{\boldsymbol{c}}
\end{aligned}
$$

$\underset{J}{J}$ is the Jacobian matrix, its components are defined as:

$$
J_{i j} \equiv \frac{\partial R_{i}}{\partial c_{j}}
$$

In this research the components of the Jacobian matrix are computed numerically through a central difference scheme:

$$
J_{i j}=\frac{R_{i}\left(c_{1}, \ldots, c_{j}+\epsilon, \ldots\right)-R_{i}\left(c_{1}, \ldots, c_{j}-\epsilon, \ldots\right)}{2 \epsilon}
$$

with $\epsilon=10^{-6}$.

The iteration begins with an estimated initial value $\underline{c}^{0}$ and continues until equation 3.35 is approximately satisfied. The iterative process stops when the $L_{2}$ norm of the residual vector satisfies the inequality $\|R\|_{2} \leq 10^{-6}$.

At each iteration of Newton's method, the linear system (equation 3.36) is solved by a LU decomposition using the frontal method proposed by Hood (1976), whose main advantage is the economy of memory. Subroutines of the 
Basic Linear Algebra Subprograms (BLAS) package are used to improve the code portability when different platforms are used. 


\section{4}

\section{Validation}

The new implemented written code must be tested for its validation. This is achieved by solving different examples which have exact analytical solution. Section 4.1 presents the solution of a suspension flow through a cylindrical tube and the predictions are compared with the exact fully developed concentration and velocity profiles, obtained by Phillips et al. (1992). In section 4.2, a similar problem is tested, but now considering the suspension flow through a channel between parallel plates.

The computational code used in this research is an extension of the program developed by Carvalho (1996) and Romero (2003), written in Fortran77/90. This program has been widely tested for Newtonian fluids in different geometries and with free surfaces. Moreover, it has also been tested for flow of dilute viscoelastic polymer solutions with free surfaces (Romero, 2003).

The program used is composed of three main parts: PREPRO (preprocessor), SOLVER (solver), and POSTPRO (post-processor). In the PREPRO, the geometry, number of regions, number of elements, type of fluid, and boundary conditions are defined. Then, the solution fields are obtained by the SOLVER. Finally, the POSTPRO generates files that can be read be the software Tecplot $360^{1}$.

The computing platform utilized to compile and execute the code was a desktop PC with the following specifications:

- Intel® Core ${ }^{\mathrm{TM}}$ i7-3770 CPU @ 3.40GHz 3.40GHz

- Installed memory: 8.00GB

- Windows 764 bits

The two examples used in this chapter presents regions at which the shear rate is zero. In the cylindrical tube and rectangular channel, this region is the

\footnotetext{
${ }^{1}$ Tecplot 360 for MS-WINDOWS.
} 
symmetry line. There, the diffusive flux model predicts concentration profiles with $c=c_{m}$, representing a singularity, which lead to numerical convergence problems. The singularity region is localized in a small zone with a characteristic length of the order of magnitude of the particle size. In this limit, the description of the suspension as a continuum medium is not valid. To avoid this problem, it is used the non-local stress concept developed by Nott \& Brady (1994) and Miller \& Morris (2006) for suspension balance model, and used by Yezaz \& Singh (2011) for diffusive flux model. The singularity is removed by adding a non-local shear rate $\left(\dot{\gamma}_{N L}\right)$, that is constant, to the local shear rate $\dot{\gamma}$ in equation 3.14. The non-local shear rate is defined as:

$$
\dot{\gamma}_{N L}=\epsilon \frac{U_{\max }}{l},
$$

where $\epsilon=(a / l)^{2}$, and $l$ is the radius of the cylindrical tube or half of the gap width of the rectangular channel.

The use of the non-local shear rate avoids particle concentration approach to maximum packing fraction.

\section{1. Flow of Suspension of Particles through a Cylindrical Tube}

The diffusive flux model has exact analytical solutions under certain hypotheses. Phillips et al. (1992) obtained predictions for the particle concentration and velocity profiles of fully developed flow through a cylindrical tube. Exact analytical solutions are obtained for values of $k_{c}$ and $k_{\eta}$ such that the ratio $\frac{k_{c}}{k_{\eta}}=0.65$. The exact analytical particle concentration profile at the cross section of a tube of radius $R$ is:

$$
c=\frac{c_{m}}{1+\frac{c_{m}-c_{W}}{c_{W}} \hat{r}}
$$

where $c_{w}$ is the particle concentration at the tube wall, and $\hat{r}=r / R$ is its dimensionless radius. 
The velocity profile is calculated by integrating the following equation:

$$
\hat{\dot{\gamma}}_{r z}(\hat{r})=\hat{r} / \eta_{r}
$$

where $\hat{\dot{\gamma}}_{r z}(\hat{r}) \equiv \dot{\gamma}_{r z}\left(2 \eta_{s} / R(d p / d z)\right)$. Equation 4.3 is integrated after substituting the value of the normalized suspension viscosity (equation 2.7), which also depends on the radial position. The velocity $\left(\hat{u}=u / U_{\max }\right)$ profile of the fullydeveloped flow is:

$$
\hat{u}=1+\int_{0}^{\hat{r}} \frac{\hat{r}}{\left(1-c / c_{m}\right)^{-1.82}} d \hat{r}
$$

The concentration and velocity profiles are compared to the numerical predictions of the $2 \mathrm{D}$ flow through a cylindrical tube. The flow domain is presented in Figure 4.1. The boundary conditions used are:

- At the inlet: Prescribed velocity profile. Constant concentration profile (average concentration), which is the so called bulk concentration, $c_{\text {bulk }}$.

- At the outlet: Developed velocity and concentration profiles, and imposed outlet pressure.

- At the wall: No-slip and no-penetration of suspension. No flux of particles.

- At the line of symmetry: No-penetration of suspension and vanishing shear stress. No-flux of particles. 


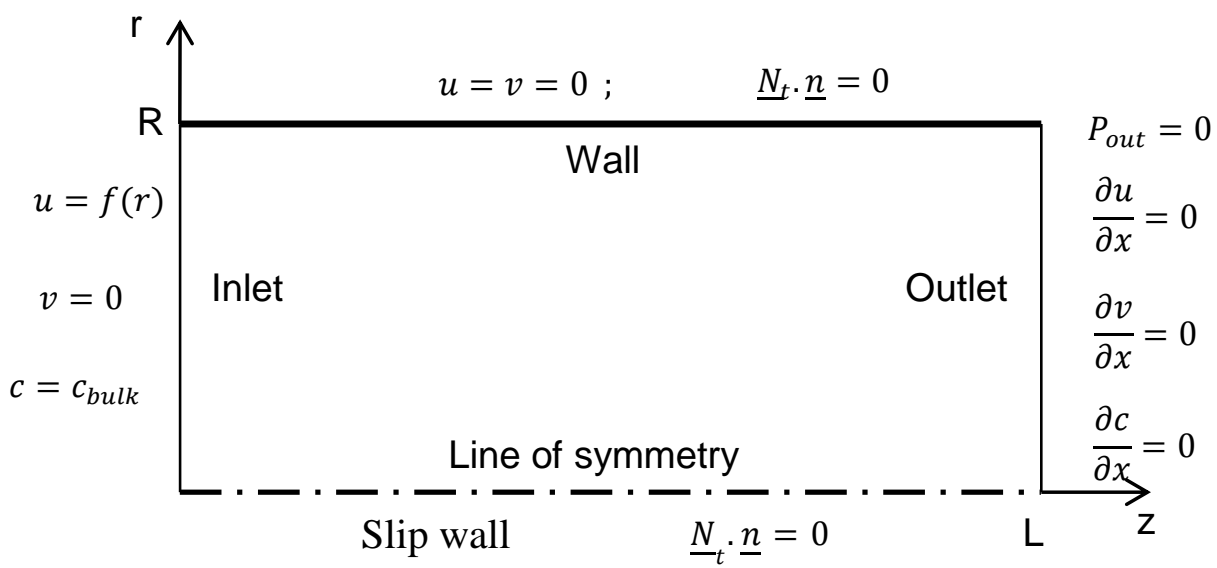

Figure 4.1: Cylindrical tube geometry and boundary conditions.

The solutions of our numerical code use the following values:

- Bulk particle concentration: $c_{\text {bulk }}=0.40$ and 0.60

- Tube radius: $R=1.0 \mathrm{~mm}$

- Tube length: $L=10.0 \mathrm{~mm}$

- Particle radius: $a=0.04 \mathrm{~mm}$

- Flow rate: $Q=100 \mathrm{~mm}^{3} / \mathrm{s}$

- Bulk viscosity: $\eta=0.054$ Pa.s

Two different meshes are used, as shown in Table 4.1, to study the influence of the mesh refinement in the accuracy of the solution.

Table 4.1: Meshes used in the tube simulation.

\begin{tabular}{|c|c|c|}
\hline Mesh name & $\mathrm{N}^{\mathbf{o}}$ elem. in axial direction & $\mathrm{N}^{\mathbf{o}}$ elem. in radial direction \\
\hline M1 & 15 & 15 \\
\hline M2 & 25 & 30 \\
\hline
\end{tabular}

\subsection{1.}

\section{Solution for Mesh M1}

Solutions were obtained for the parameter values shown in Table 4.2.

The ratio $k_{c} / k_{\eta}$ was kept constant and equal to $k_{c} / k_{\eta}=0.65$, to make possible comparisons with the analytical solution presented by Phillips et al. (1992). 
Table 4.2: Data for the simulation for mesh M1.

\begin{tabular}{|c|c|c|c|c|c|}
\hline Case & $c_{\text {bulk }}$ & $k_{c}$ & $k_{\eta}$ & $\mathrm{N}^{\circ}$ Elem & DoF \\
\hline tubes4-005 & 0.40 & $\mathbf{6 0 . 0}$ & $\mathbf{9 2 . 0}$ & 225 & 4582 \\
\hline tubes4-006 & 0.40 & $\mathbf{2 0 0}$ & $\mathbf{3 0 5}$ & 225 & 4582 \\
\hline tubes6-004 & 0.60 & $\mathbf{6 . 0}$ & $\mathbf{9 . 2}$ & 225 & 4582 \\
\hline tubes6-006 & 0.60 & $\mathbf{6 0 . 0}$ & $\mathbf{9 2 . 0}$ & 225 & 4582 \\
\hline
\end{tabular}

Flow of suspension with $c_{b u l k}=0.40$ :

Figure 4.2 shows the value of particle concentration as a function of the axial coordinate along the centerline of the tube. It is observed that the concentration in the centerline starts as the imposed bulk concentration value $\left(c_{\text {bulk }}=0.4\right)$, then the migration begins until a developed state is reached, in which the concentration and velocity profiles are independent of the axial coordinate. Because of its higher values of the diffusion parameters $k_{c}$ and $k_{\eta}$, the fully developed value is reached before for the case labeled tubes4-006.

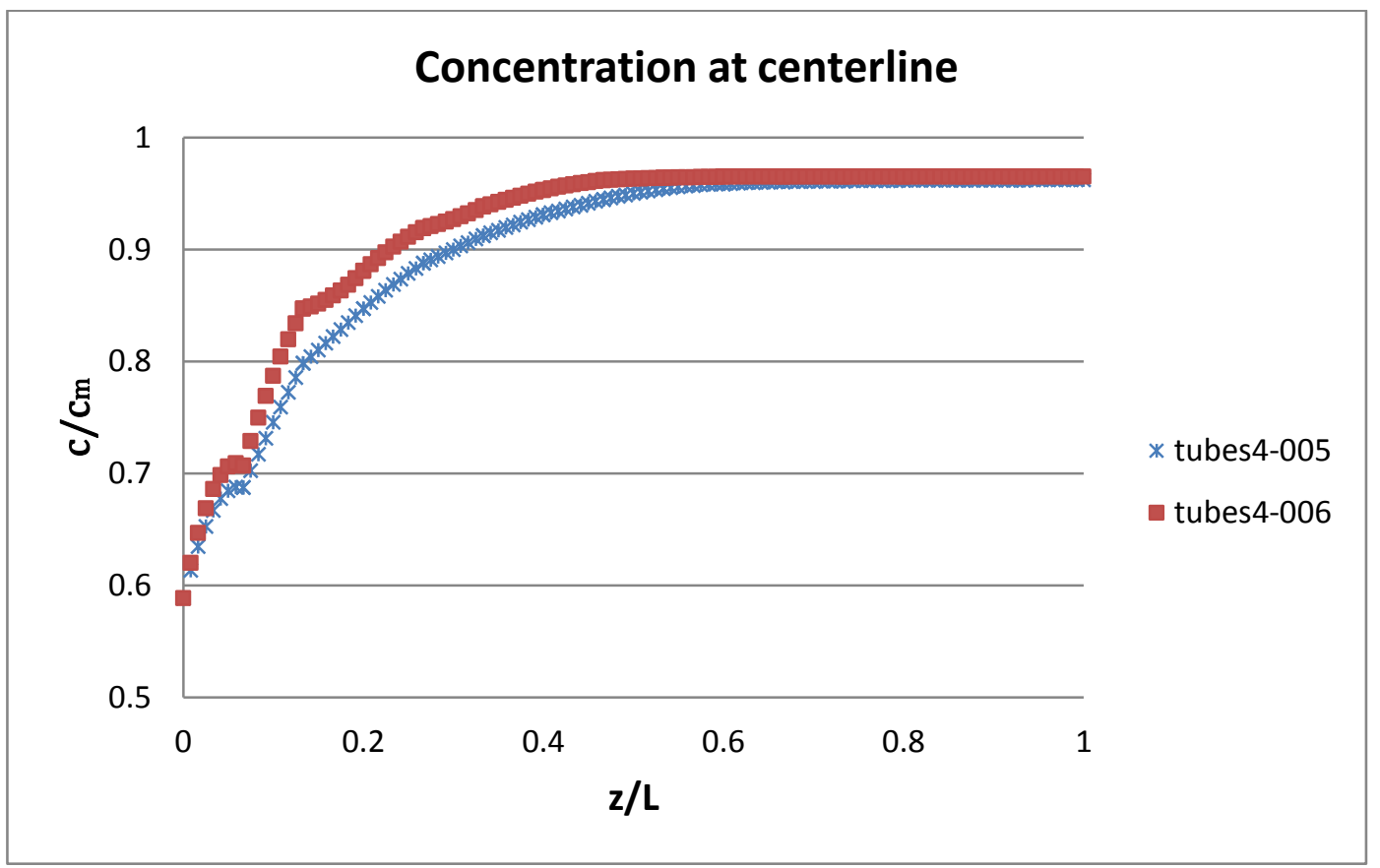

Figure 4.2: Evolution of particle concentration along the centerline. $c_{b u l k}=0.40$. 
It can be observed in Figure 4.2 that due to high concentration gradients near the inlet, the concentration curve is not smooth. A better description of particle migration can be made by mesh refinement near this region.

The fully-developed concentration profile is presented in Figure 4.3. The plot also shows the exact analytical solution. The agreement between the numerical predictions and exact solution is excellent. The simulation case labeled "tubes4-005" presents a mean absolute percentage error of $1.5 \%$, while the other case ("tubes4-006") this same error is $1.9 \%$. The maximum absolute percentage error is $3.5 \%$, found at the tube centerline, for the case labeled "tubes4-006", showing that the deviation is localized.

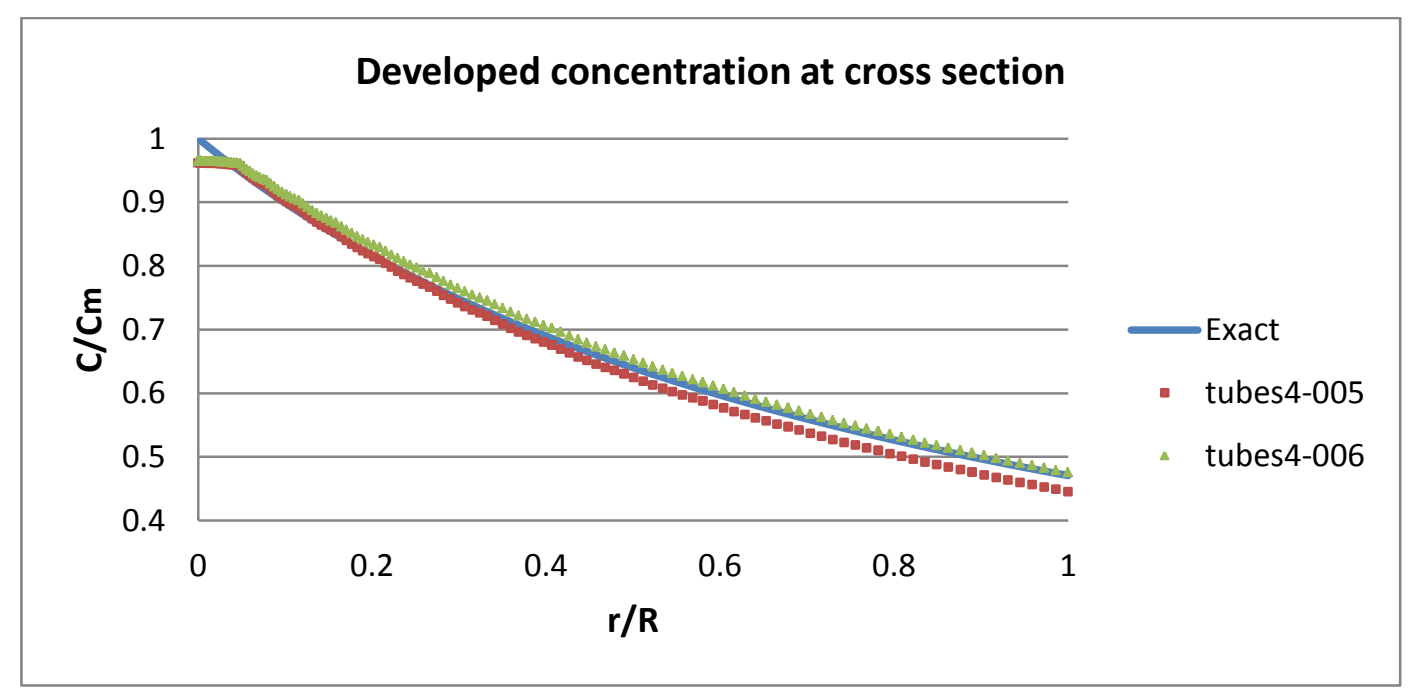

Figure 4.3: Comparison of particle concentration: numerical vs. exact solutions. $c_{\text {bulk }}=$ 0.40 .

The small discrepancy observed near the centerline is associated with the non-local stress concept used to remove the singularity at the symmetry line. This region can be minimized by using a very fine mesh near the centerline.

The corresponding fully-developed velocity profile prediction is shown in Figure 4.4 together with the analytical solution. An excellent agreement is observed. Because of the high concentration (then high viscosity) near the symmetry line, the velocity profile approaches a constant value near the axis. 


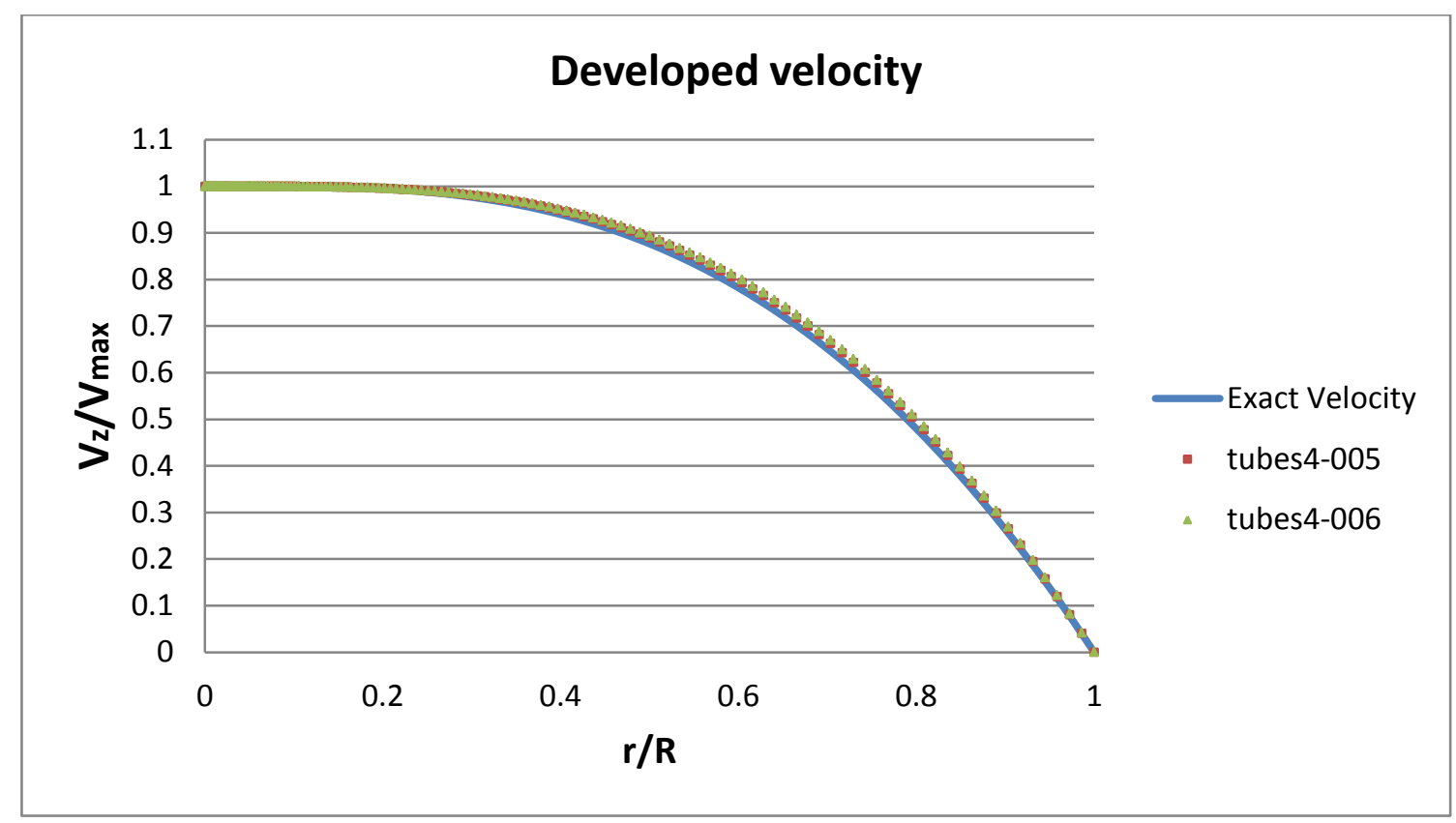

Figure 4.4: Developed velocity of the suspension at the end of the tube. $c_{\text {bulk }}=$ 0.40 .

The complete concentration field is shown in Figure 4.5 for $c_{b u l k}=0.4$. The evolution towards a fully developed situation is clearly observed.
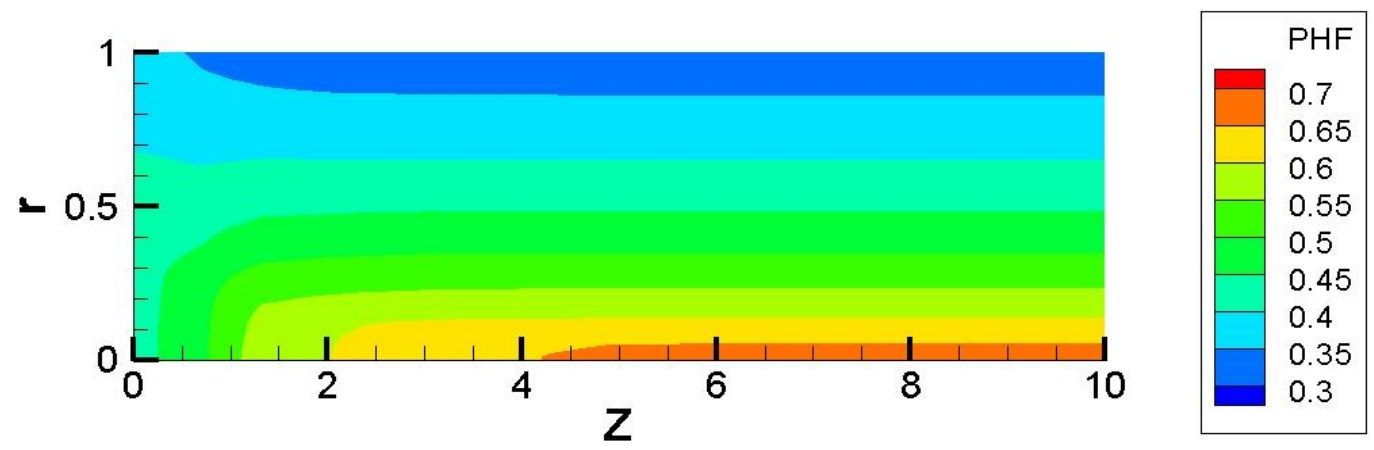

Figure 4.5: Project "tubes4-006". 2D flow of suspension in a cylindrical tube. $c_{b u l k}=0.40$.

Flow of suspension with $c_{\text {bulk }}=0.60$ :

Figure 4.6 presents the values of particle concentration as a function of the axial coordinate along the centerline of the tube. It is observed that the concentration in the centerline starts as the imposed bulk concentration value $\left(c_{\text {bulk }}=0.6\right)$, then the migration begins until it is reached a fully developed value, in which the concentration profile is independent of the axial coordinate. Case 
"tubes6-006" reaches its developed concentration profile before case "tubes6004", which has lower values of the migration constants $k_{c}$ and $k_{\eta}$.

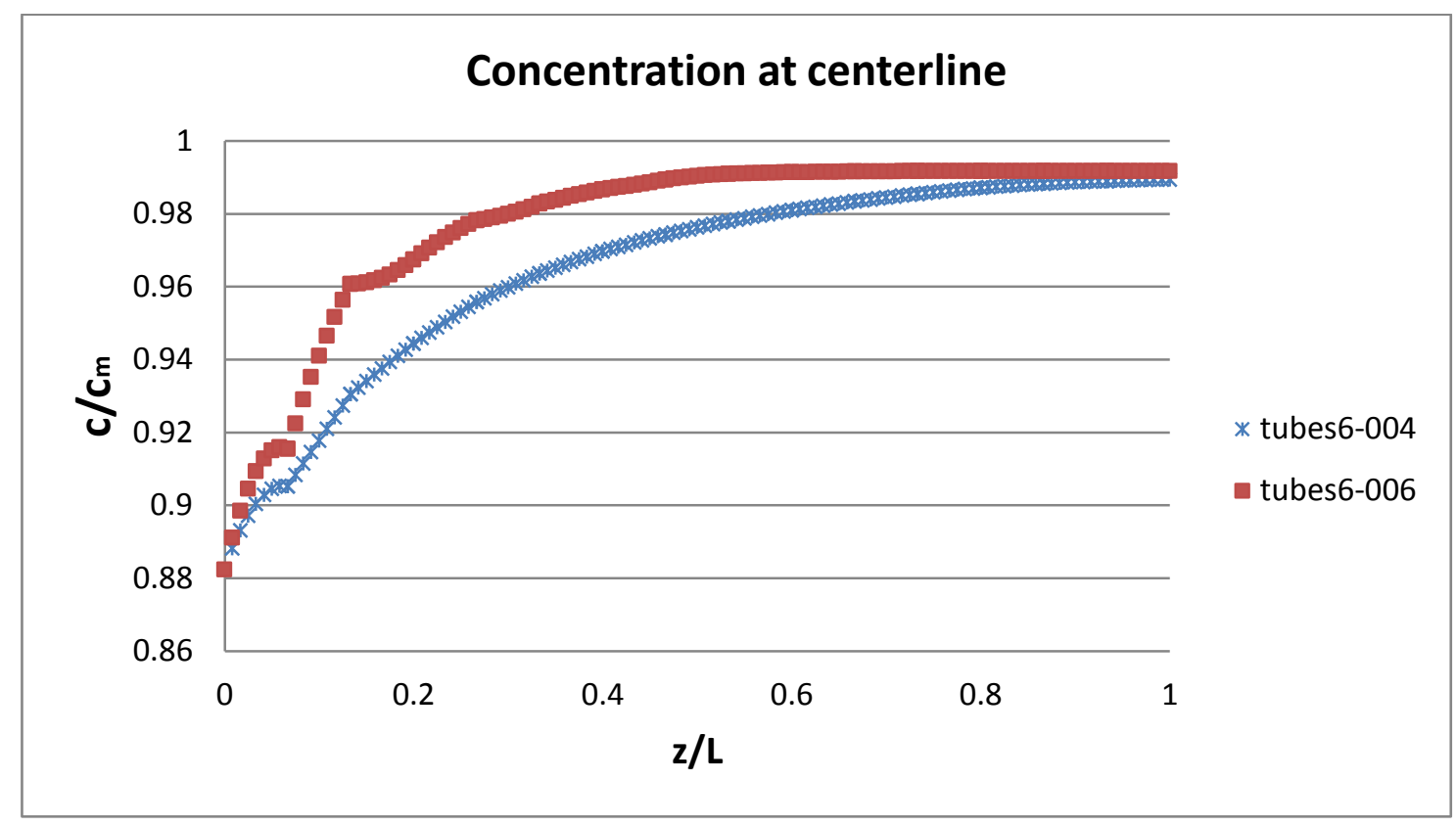

Figure 4.6: Evolution of particle concentration along the centerline. $c_{b u l k}=0.60$.

The fully developed concentration profile for both cases is plotted together with the exact solution in Figure 4.7. A high discrepancy is observed for case labeled "tubes6-004", because the tube is too short, so that particles could not migrate to reach their developed concentration profile. The simulation case labeled "tubes6-004" presents a mean absolute percentage error of $0.7 \%$, while the other case ("tubes6-006") this same error is $0.4 \%$. The maximum absolute percentage error is $0.8 \%$, found at the tube centerline, for the case labeled "tubes4-006", showing that the deviation is localized. 


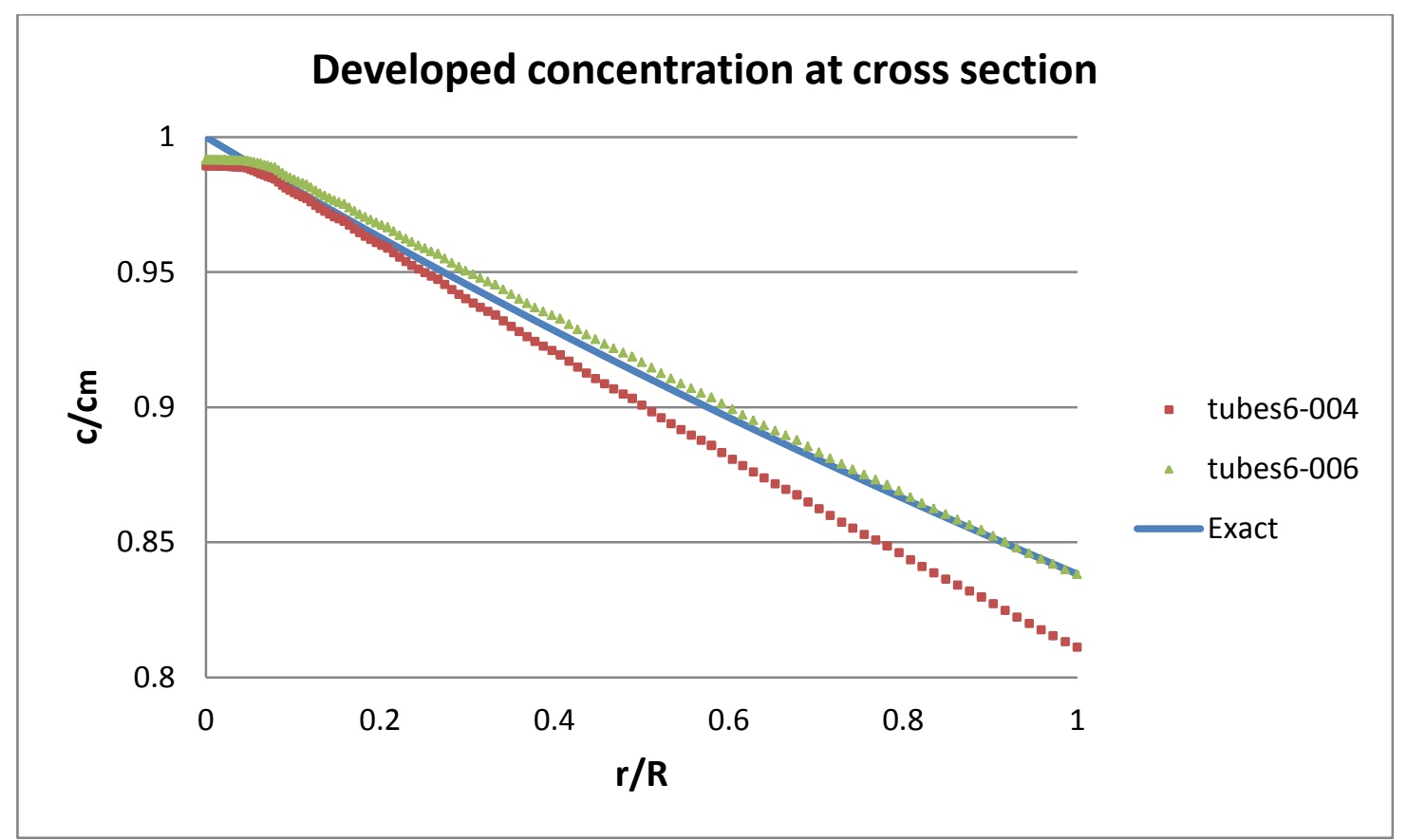

Figure 4.7: Comparison of particle concentration: numerical vs. exact solutions. $c_{b u l k}=$ 0.60 .

Comparison of the developed velocity of the suspension at the end of the tube with the exact solution is shown in Figure 4.8. An excellent agreement with the analytical solution is observed. It can be observed that velocity profiles converge to exact velocity faster than concentration profiles. The mean absolute percentage error for both cases is below $0.3 \%$.

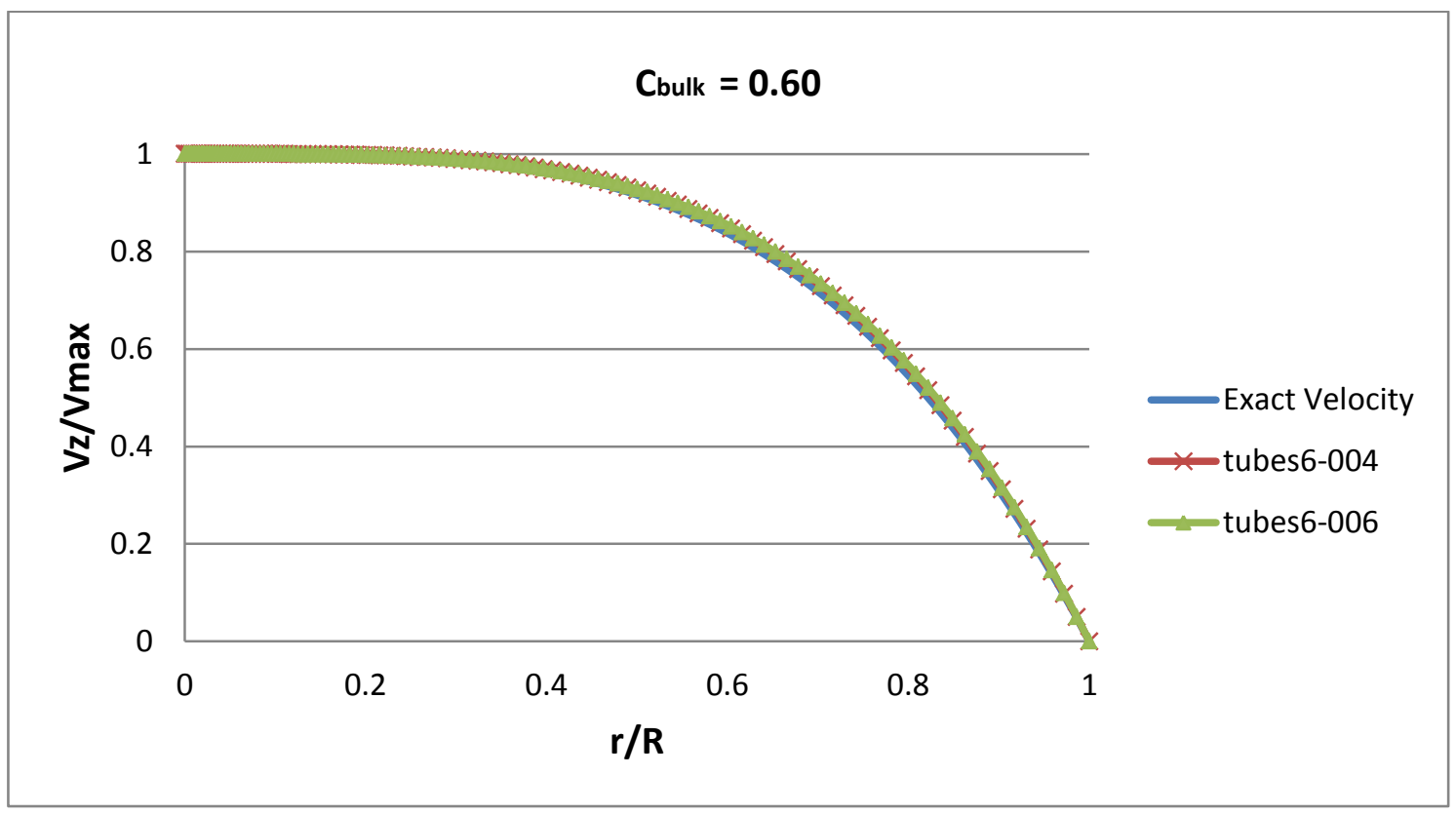

Figure 4.8: Developed velocity of the suspension at the end of the tube. $c_{b u l k}=0.60$. 
Finally, Figure 4.9 depicts a plot of the suspension flow, with $c_{b u l k}=0.6$, in two-dimensions.
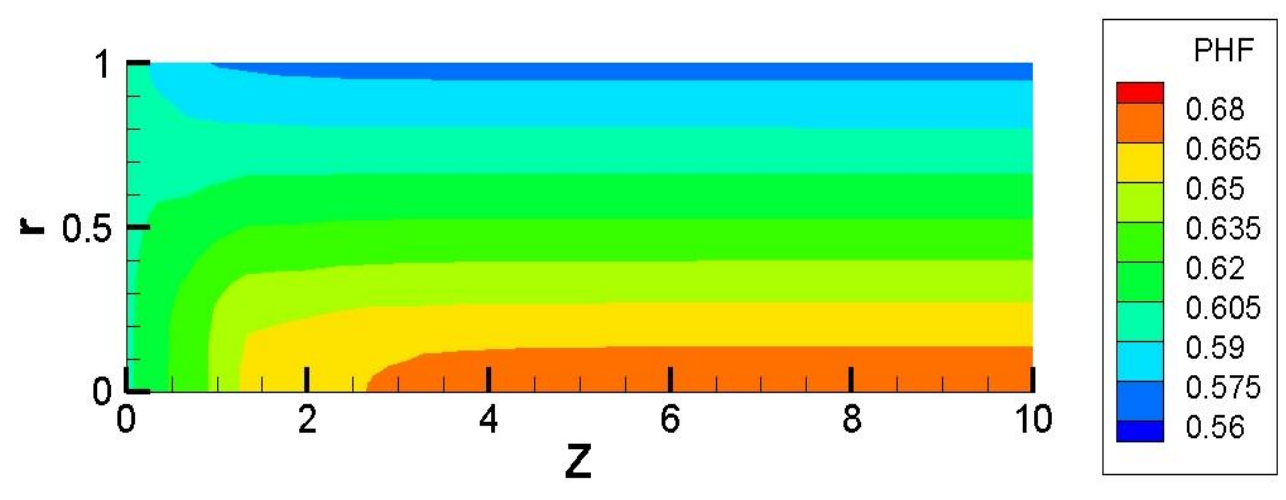

Figure 4.9: Project "tubes6-006". 2D flow of suspension in a cylindrical tube. $c_{b u l k}=0.60$.

\subsection{2.}

\section{Solution for Mesh M2}

Solutions with a finer mesh were obtained for some of the cases discussed the previous sub-sections. The values of the parameters are shown in Table 4.3.

Table 4.3: Data for the simulation for mesh M2.

\begin{tabular}{|c|c|c|c|c|c|}
\hline Cases & $c_{\text {bulk }}$ & $k_{c}$ & $k_{\eta}$ & N $^{\text {o }}$ Elem & DoF \\
\hline tubes4-014 & 0.40 & $\mathbf{2 0 0}$ & $\mathbf{3 0 5}$ & 750 & 14807 \\
\hline tubes6-007 & 0.60 & $\mathbf{6 0}$ & $\mathbf{9 2}$ & 750 & 14807 \\
\hline
\end{tabular}

Flow of suspension with $c_{b u l k}=0.40$ :

Comparison of simulations for the two meshes is plotted, so that the cases compared are "tubes4-006" (Mesh 1) and "tubes4-014" (Mesh 2). Figure 4.10 shows the value of particle concentration as a function of the axial coordinate along the centerline of the tube for two cases. It is observed that the concentration in the centerline starts with the bulk concentration value $\left(c_{b u l k}=0.4\right)$, then the migration begins until it is reached a developed state, in which the concentration profile is independent of the axial coordinate. 
Solution for a the finer mesh present a smoother curve near the inlet of the tube, besides developed particle concentration at the centerline is closer to the maximum packing concentration.

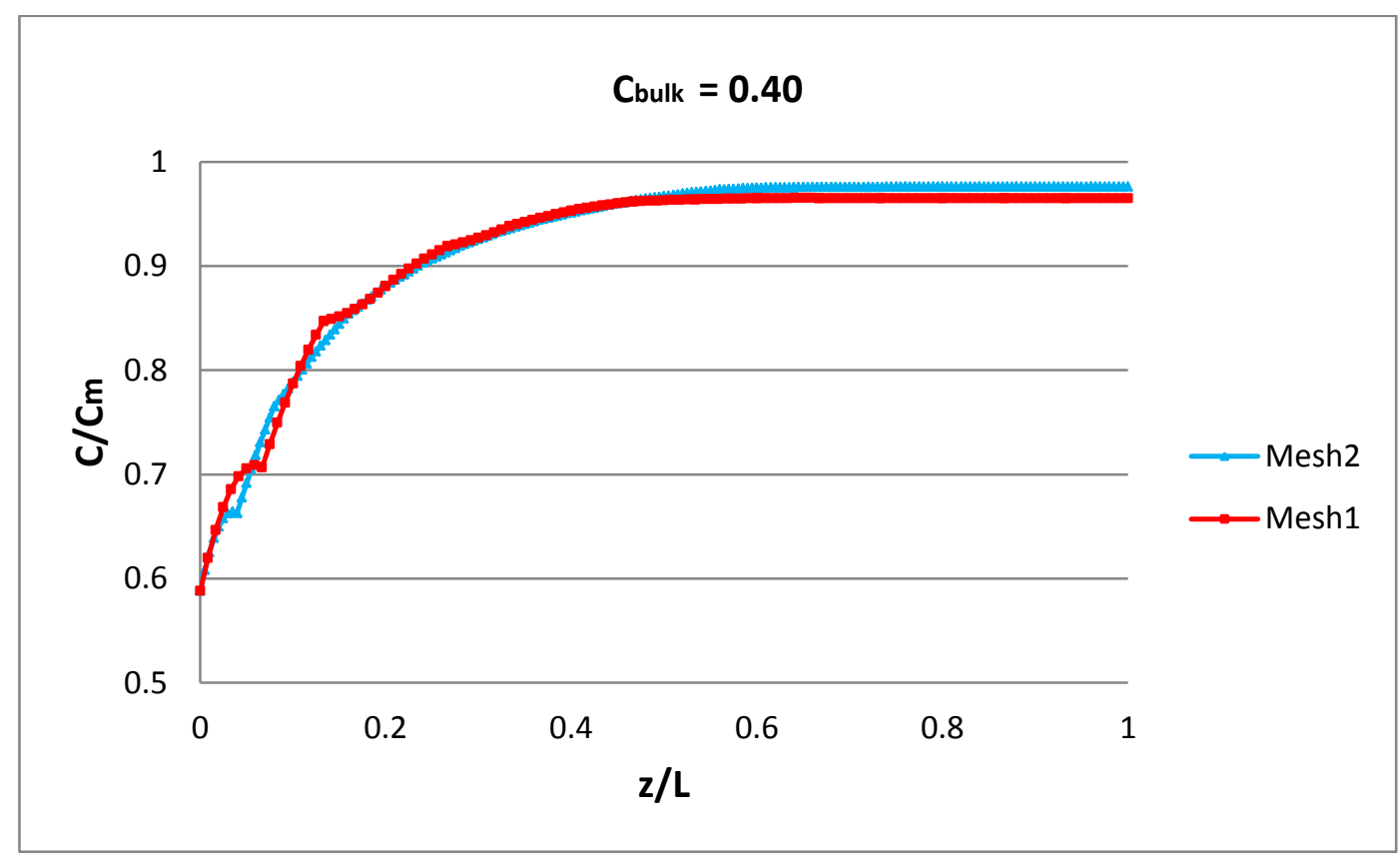

Figure 4.10: Evolution of particle concentration along the centerline for the two meshes. $c_{\text {bulk }}=0.40$.

The fully developed concentration profiles are presented in Figure 4.11, for the two meshes. It can be observed that solution with mesh M2 has better agreement than solution with mesh M1. Velocity profile comparison was omitted due to virtually equal values. The mean absolute percentage error for mesh M2 is $0.8 \%$, with maximum absolute percentage error of $2.4 \%$ localized at the tube centerline, showing that the deviation is localized. 


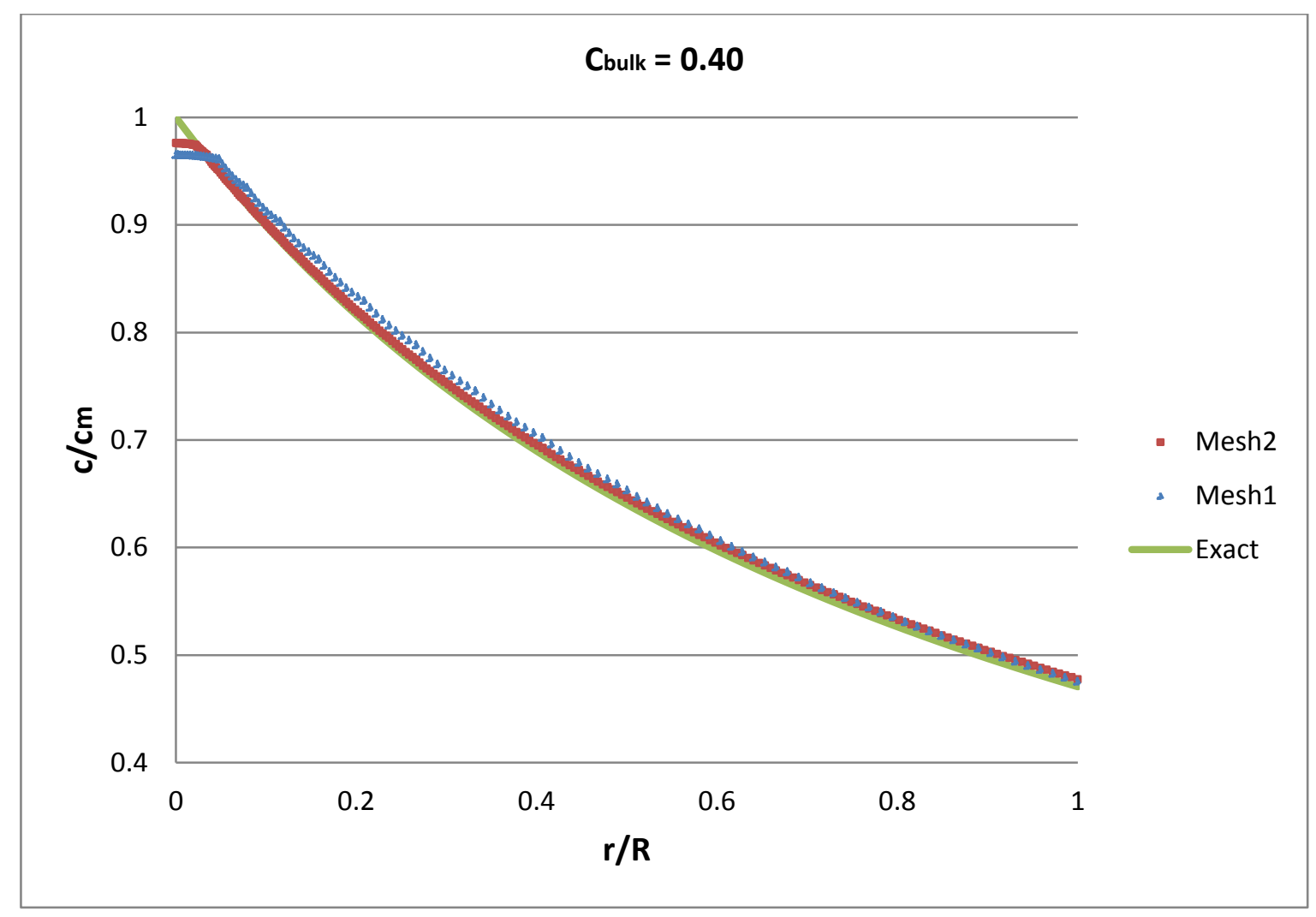

Figure 4.11: Comparison of particle concentration: numerical vs. exact solutions. $c_{b u l k}=0.40$.

\section{Flow of suspension of $c_{b u l k}=0.60$ :}

It is also compared simulations for the two meshes: "tubes6-006" (Mesh 1) and "tubes6-007" (Mesh 2).

Figure 4.12 shows the value of particle concentration as a function of the axial coordinate along the centerline of the tube for two cases. It is observed that the concentration in the centerline starts with the bulk concentration value $\left(c_{\text {bulk }}=0.6\right)$, then the migration begins until it is reached a developed state, in which the concentration profile is independent of the axial coordinate.

Again, solution for a the finer mesh present a smoother curve near the inlet of the tube, besides developed particle concentration at the centerline is closer to the maximum packing concentration. 


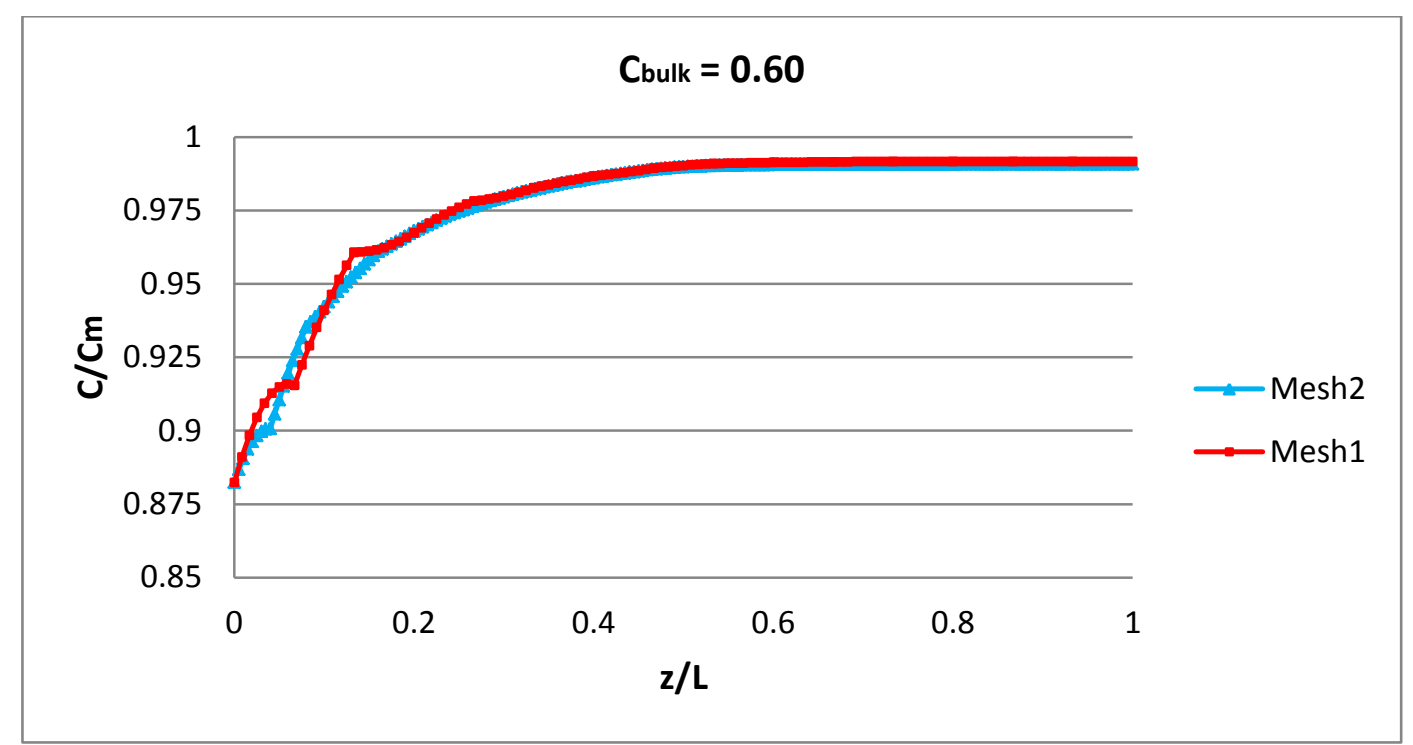

Figure 4.12: Evolution of particle concentration along the centerline. $c_{b u l k}=0.60$.

The fully developed concentration profiles are presented in Figure 4.13, for the two meshes. It can be observed that solution with mesh M2 has better agreement than solution with mesh M1. The mean absolute percentage error for mesh M1 is $0.4 \%$ while for mesh M2 is $0.07 \%$. The maximum absolute percentage error of mesh $\mathrm{M} 2$ is $0.9 \%$ at the centerline tube, showing that the error is localized.

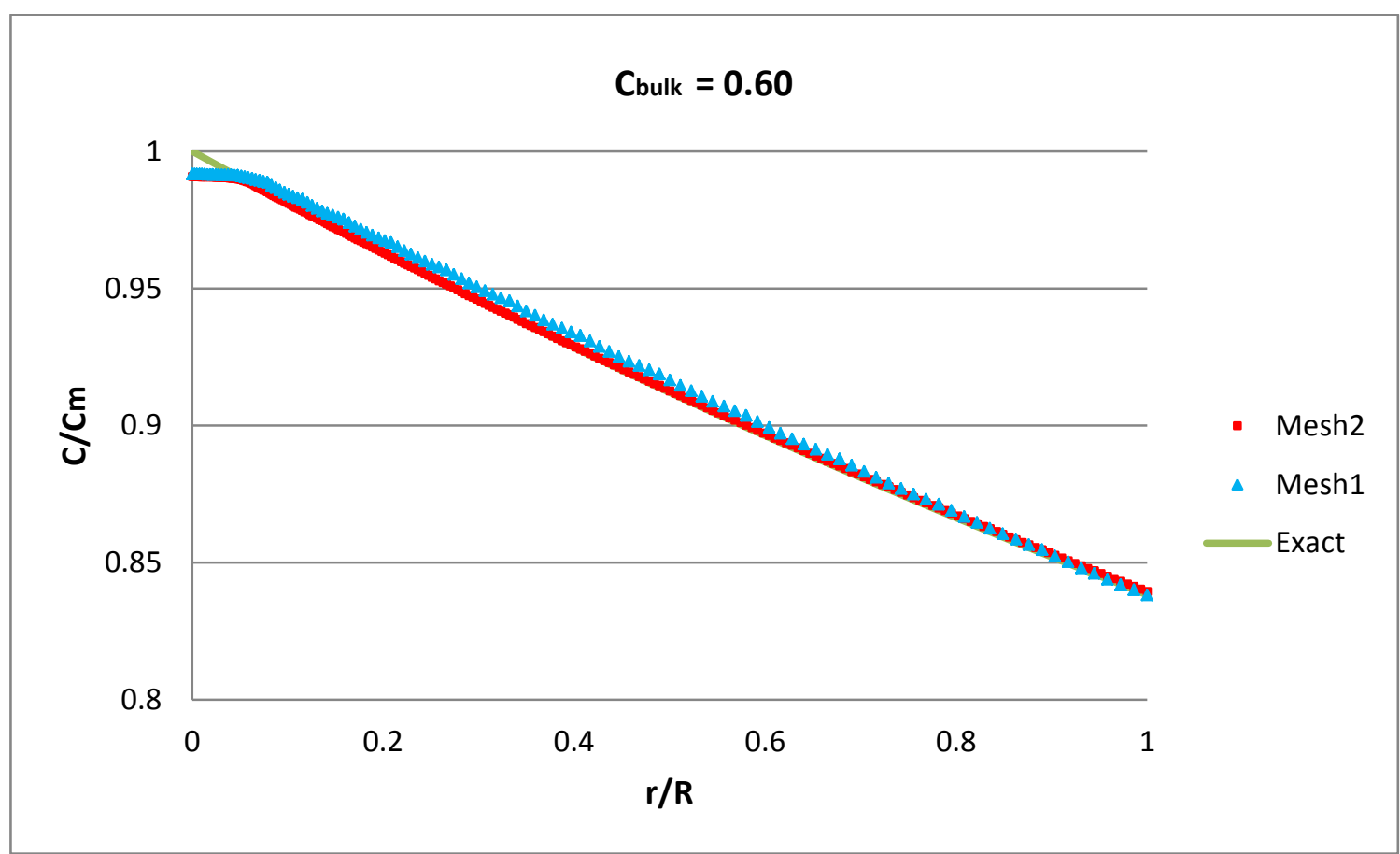

Figure 4.13: Comparison of particle concentration: numerical vs. exact solutions. $c_{\text {bulk }}=$ 0.60 . 


\section{2.}

\section{Flow of Suspension of Particles through a Rectangular Channel}

The same hypotheses, adopted for cylindrical tube, are made to obtained exact analytical solutions for the rectangular channel. It is presented the analytical solution for the particle concentration and velocity profiles of a developed flow through a rectangular channel.

Exact analytical solutions are obtained for values of $k_{c}$ and $k_{\eta}$ that keep the same ratio $\frac{k_{c}}{k_{\eta}}=0.65$. The particle concentration profile in a channel of half gap width $H$ is:

$$
c=\frac{c_{m}}{1+\frac{c_{m}-c_{W}}{c_{W}} \hat{y}}
$$

where $c_{w}$ is the particle concentration at the tube wall, and $\hat{y}=y / H$ is its dimensionless width.

The velocity profile is calculated by integrating the following equation:

$$
\hat{\dot{\gamma}}_{y x}(\hat{r})=\hat{y} / \eta_{r}
$$

where $\hat{\dot{\gamma}}_{y x}(\hat{y}) \equiv \dot{\gamma}_{y x}\left(2 \eta_{s} / H(d p / d x)\right)$. The equation 4.6 is integrated substituting the value of the normalized suspension viscosity (equation 2.7), which also depends on the transversal position. The velocity profile results in:

$$
\hat{u}=1+\int_{0}^{\hat{y}} \frac{\hat{y}}{\left(1-c / c_{m}\right)^{-1.82}} d \hat{y}
$$

The concentration and velocity profiles are compared to the numerical predictions of the 2D flow through the rectangular channel. The flow domain is presented in Figure 4.14, together with the boundary conditions. 


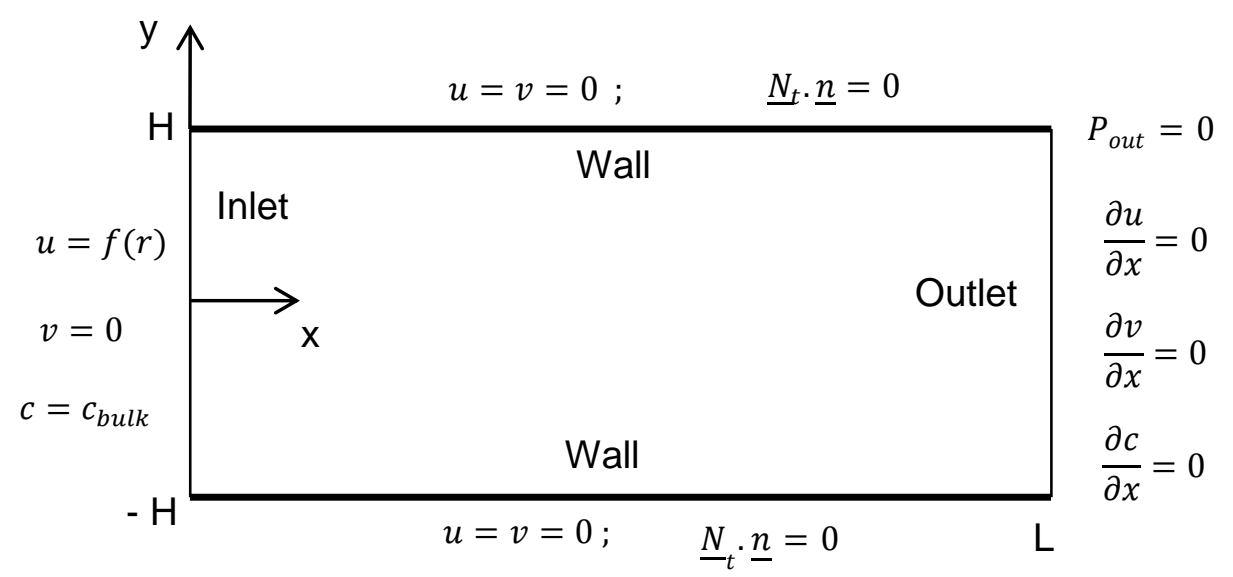

Figure 4.14: Two-dimensional rectangular channel geometry and boundary conditions.

The solutions of our numerical code use the following values:

- Bulk particle concentration: $c_{b u l k}=0.40$

- Half of the channel width: $H=0.5 \mathrm{~mm}$

- Channel length: $L=10.0 \mathrm{~mm}$

- Particle radius: $a=0.02 \mathrm{~mm}$

- Flow rate: $Q=10 \mathrm{~mm}^{2} / \mathrm{s}$

- Bulk viscosity: $\eta=0.054$ Pa.s

The simulation performed has the following values as shown in Table 4.4.

Table 4.4: Data for the simulation. Rectangular channel.

\begin{tabular}{|c|c|c|c|c|c|}
\hline Case & $c_{\text {bulk }}$ & $k_{c}$ & $k_{\eta}$ & $\mathrm{N}^{\circ}$ Elem & DoF \\
\hline channels4-006 & 0.40 & $\mathbf{0 . 9 1}$ & $\mathbf{1 . 4 1}$ & 400 & 8007 \\
\hline
\end{tabular}

Different simulations were carried out, each of them increasing the values of $k_{c}$ and $k_{\eta}$ but maintaining the same ratio $\left(\frac{k_{c}}{k_{\eta}}=0.65\right)$, value at which is calculated the exact analytical solution. It is presented the concentration and velocity profiles for just one case (“channels4-006").

Figure 4.15 shows the value of particle concentration as a function of the axial coordinate along the wall of the channel. Concentration at the wall starts with the bulk concentration value $\left(c_{\text {bulk }}=0.4\right)$, then the migration begins until a developed state is reached, in which the concentration profile is independent of 
the axial coordinates. Developed state is approximately reached at the middle of the channel length.

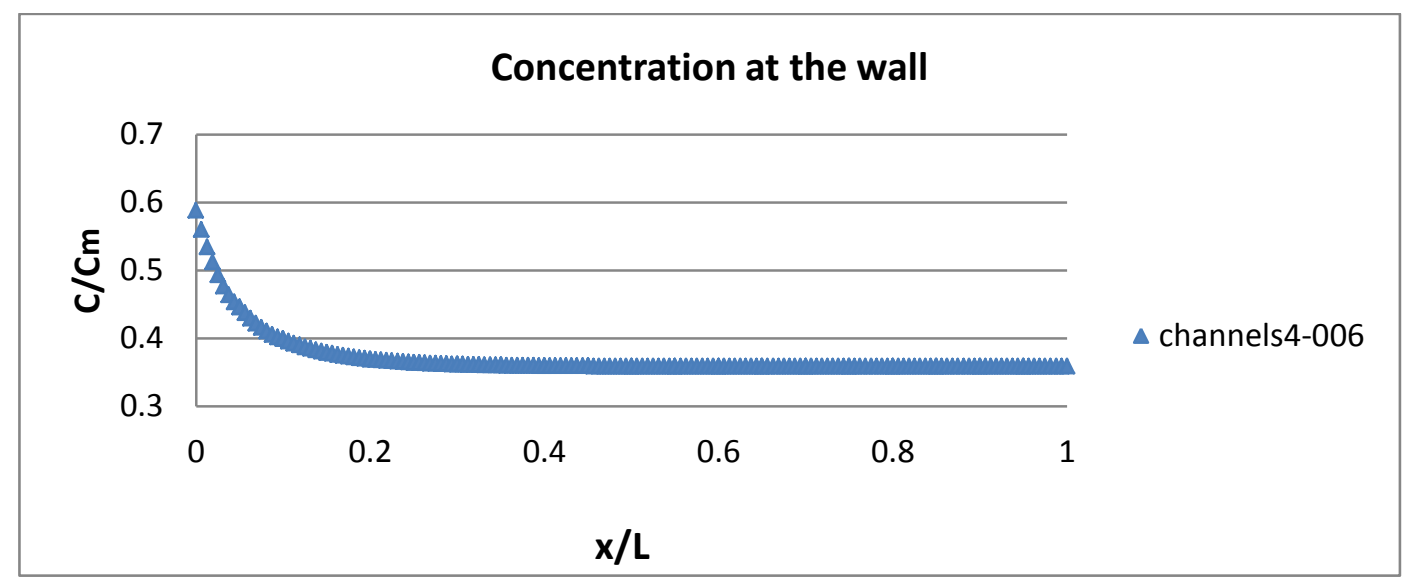

Figure 4.15: Evolution of particle concentration along the centerline. $c_{b u l k}=0.40$.

The fully-developed concentration profile is presented in Figure 4.16 together with the analytical solution. The agreement between the numerical prediction and exact solution is excellent. The mean absolute percentage error is $1.1 \%$ and the maximum absolute percentage error is $3.4 \%$ at the channel centerline. The discrepancy is associated with the non-local stress concept used to remove the singularity at the symmetry line.

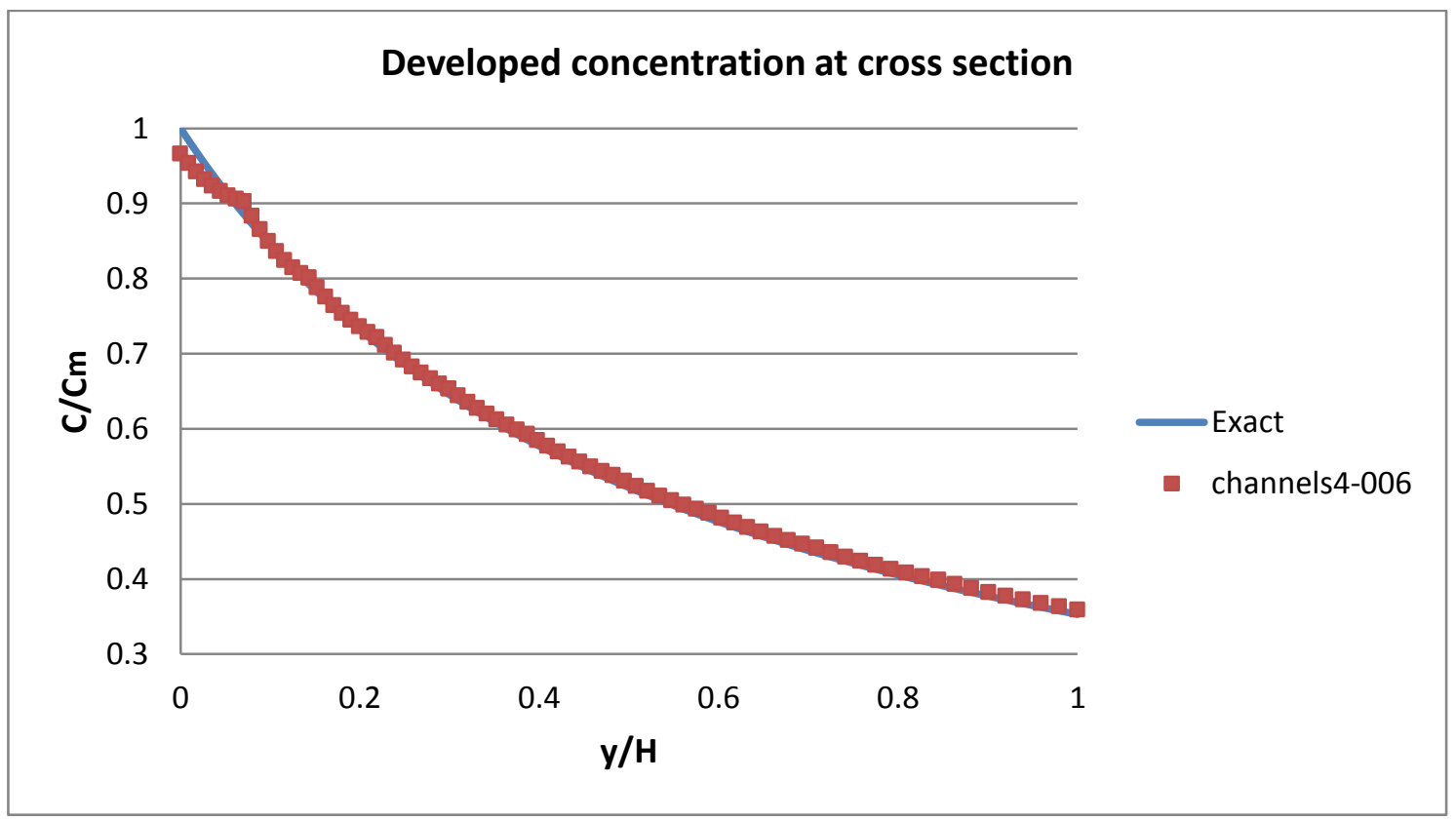

Figure 4.16: Comparison of particle concentration: numerical vs. exact solutions. $c_{b u l k}=$ 0.40 . 
The corresponding fully-developed velocity profile prediction is shown in Figure 4.17 together with the analytical solution. Because of the high concentration near the symmetry line, it is also observed a blunt in the velocity profile near the centerline.

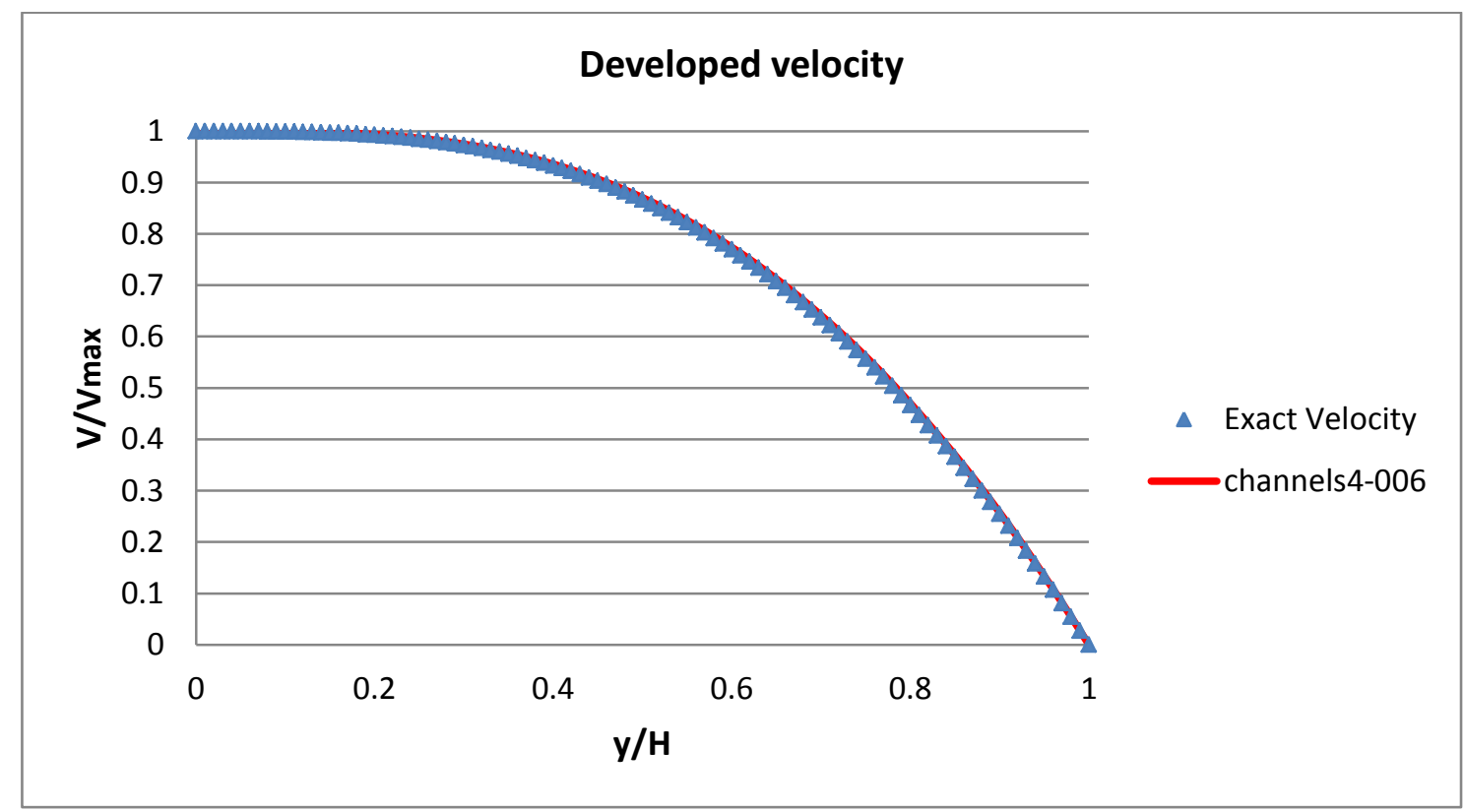

Figure 4.17: Developed velocity of the suspension at the end of the tube. $c_{b u l k}=0.40$.

The complete concentration field is shown in Figure 4.18 for $c_{b u l k}=0.6$. The evolution towards a fully developed situation is clearly observed.
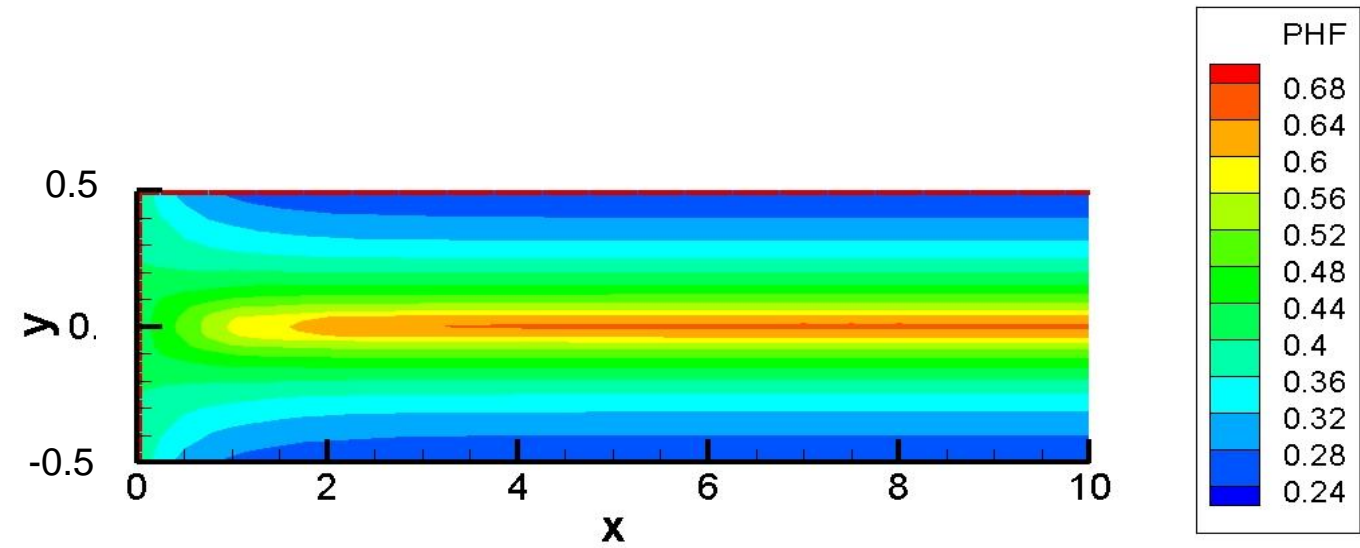

Figure 4.18: Project "tubes6-006". 2D flow of suspension in a cylindrical tube. $c_{\text {bulk }}=$ 0.40 . 


\section{3.}

\section{Closure}

The GFEM solution of the two-dimensional diffusive flux model was implemented in a homemade code. The results obtained for a suspension flow in a cylindrical tube and rectangular channel have an excellent agreement with the exact analytical solutions, for both concentration and velocity profiles, in the limit of fully developed flow.

The exact concentration profile (Phillips et al., 1992), according to the model, has a maximum value at the center line, where the shear rate vanishes, leading to zero flux of particles in that region. The numerical solution implemented here can approach this feature, through mesh refinement. It is obvious that, in order to have the numerical results converge to the exact solution in the vicinity of this singular point, the local mesh resolution must be infinitely high (Zhang \& Acrivos, 1994).

Nevertheless, it can be concluded that the numerical solution for the developed velocity profile has an excellent agreement with the exact analytical solution.

Finally, the results of this chapter show that the suspension of particles changes deeply the flow field in both the tube and the rectangular channel. The velocity profiles show a plug flow morphology in the central region which is different from the well-known parabolic profile of Newtonian fluids in laminar regimes. This flow morphology was actually observed in several experiments (karnis et al. (1966); Leighton, 1988; Zhang \& Acrivos, 1994; Altobelli et al., 1991; Lyon \& Leal, 1995) and it is an indicator that the model is capturing the main features of this flows. 


\section{Results}

5.1.

Overview

Simulations of slot coating process with particle suspension as the coating liquid is presented in this chapter. The continuity, momentum and particle transport equations were solved, using the diffusive flux model.

The main goal of the analysis was to study the effect of particle migration on the flow field. Predictions show regions of high and low particle concentration, concentration profile on the final deposited film and interface shapes at different conditions.

The physical and reference domains, where the equations are solved, are presented in Figure 5.1. 


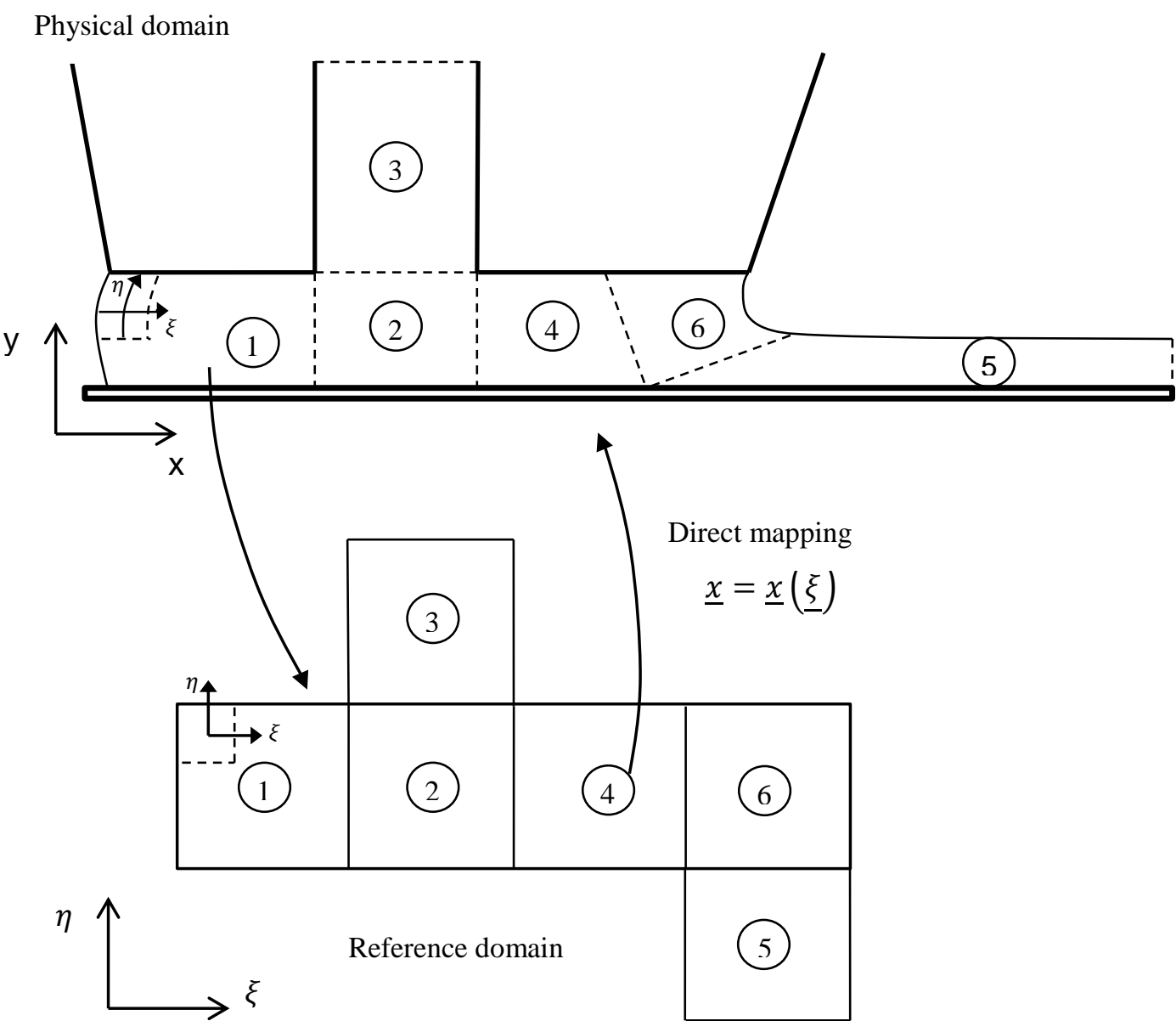

Figure 5.1: Physical domain divided in 6 regions and mapped into a reference computational domain where the equations are solved.

All predictions were in the limit of Stokes flow, i.e. vanishing Reynolds number: $R e=0$.

As explained in chapter 2 and 3, the continuity, momentum, transport, mesh, and interpolated velocity gradient equations are solved by the GFEM, with diffusive flux model as constitutive equation for particle migration. The set of resulting non-linear algebraic equations is solved using the Newton's method. This method converges quadratically when the initial guess is close the solution. This problem is highly nonlinear; therefore, obtaining the solution requires following several steps, as described below:

i. An initial guess for the free boundary problem is constructed by solving a Newtonian flow in a slot coating geometry, but replacing the two interfaces with slip walls. By removing the unknown boundary the problem becomes linear and therefore Newton's method converges in one iteration. 
ii. Progressively the slip walls are substituted by free surfaces with high surface tension, to avoid highly curved shapes. This constitutes the solution of slot coating flow with Newtonian liquid.

iii. The result of step two is used as an initial guess for the flow of particle suspension, but first without particle migration, i.e. the particle transport coefficients are set to zero. Two new equations are solved: transport equation and interpolated velocity gradient. The solution calculates a new field, the concentration field, which is constant. Moreover, the interpolated velocity gradient field is computed.

iv. Finally the solution of step iii. is used as an initial guess for suspension flow with particle migration. This is performed increasing the values of the constants: $k_{c}$ and $k_{\eta}$.

An analysis of suspension flow in the feed slot is presented in section 5.2, in order to determine the adequate mesh refinement. In section 5.3, the general characteristics of the concentration field in slot coating is presented, showing the regions of higher and lower concentration. After that, the influence of film thickness on the particle distribution is presented in section 5.4. Finally, section 5.5 presents the particle concentration profile at final coated layer for different film thicknesses.

\section{2.}

\section{Particle Migration in the Feed Slot}

The first analysis was limited to the flow through the feed slot. The diffusion parameters $\left(k_{c}\right.$ and $\left.k_{\eta}\right)$ were such that the particle concentration profile at the end of the feed slot reaches the fully developed profile. Since the fully developed profile is known, the predictions were used to verify the accuracy obtained with different mesh refinement.

The domain is presented in Figure 5.2, together with the boundary conditions. This is the same problem from the validation chapter (section 4.2). The parameters considered in the analysis of suspension flow through the feed slot were: 
- Feed slot gap: $\quad H_{s}=0.1 \mathrm{~mm}$

- Feed slot length: $\quad L=0.6 \mathrm{~mm}$

- Particle radius: $\quad a=0.004 \mathrm{~mm}$

- Flow rate: $\quad q=100 \mathrm{~mm}^{2} / \mathrm{s}$

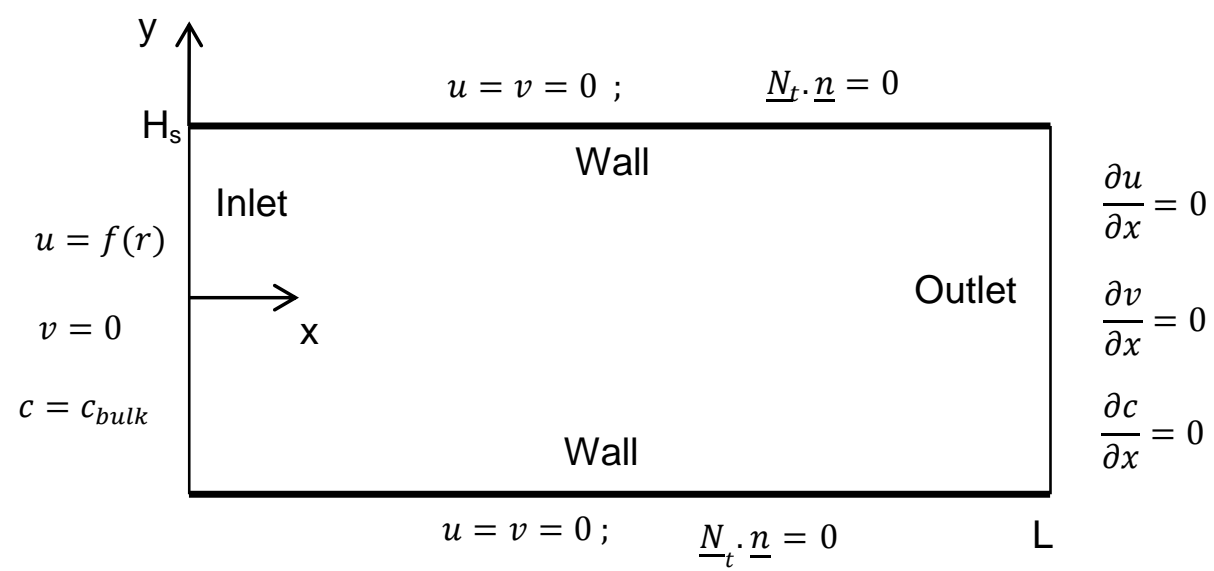

Figure 5.2: Feed slot domain.

Simulations are performed for two meshes as describes in Table 5.1.

Table 5.1: Mesh uses in the feed slot suspension flow.

\begin{tabular}{|c|c|c|}
\hline Mesh name & N $^{\mathbf{o}}$ of elem. $\mathrm{y}$ - direction & $\mathrm{N}^{\mathbf{o}}$ of elem. $\mathrm{x}$ - direction \\
\hline M1 & 8 & 14 \\
\hline M2 & 12 & 21 \\
\hline
\end{tabular}

Different simulations were carried out, with different values of $k_{c}$ and $k_{\eta}$, but maintaining the same ratio $\left(\frac{k_{c}}{k_{\eta}}=\frac{2}{3}\right)$. The parameters explored are presented in Table 5.2.

Table 5.2: Relevant variables for each case.

\begin{tabular}{|c|c|c|c|c|c|}
\hline Case & Mesh & $c_{\text {bulk }}$ & $k_{c}$ & $k_{\eta}$ & $\mathrm{N}^{\circ}$ Elem \\
\hline slotm1s4-005 & M1 & 0.40 & $\mathbf{0 . 2}$ & $\mathbf{0 . 3}$ & 112 \\
\hline slotm2s4-006 & M2 & 0.40 & $\mathbf{0 . 6}$ & $\mathbf{0 . 9}$ & 252 \\
\hline
\end{tabular}

The concentration profiles at the outlet of the rectangular channel are presented in Figure 5.3, together with the analytical solution of the fully developed flow. 


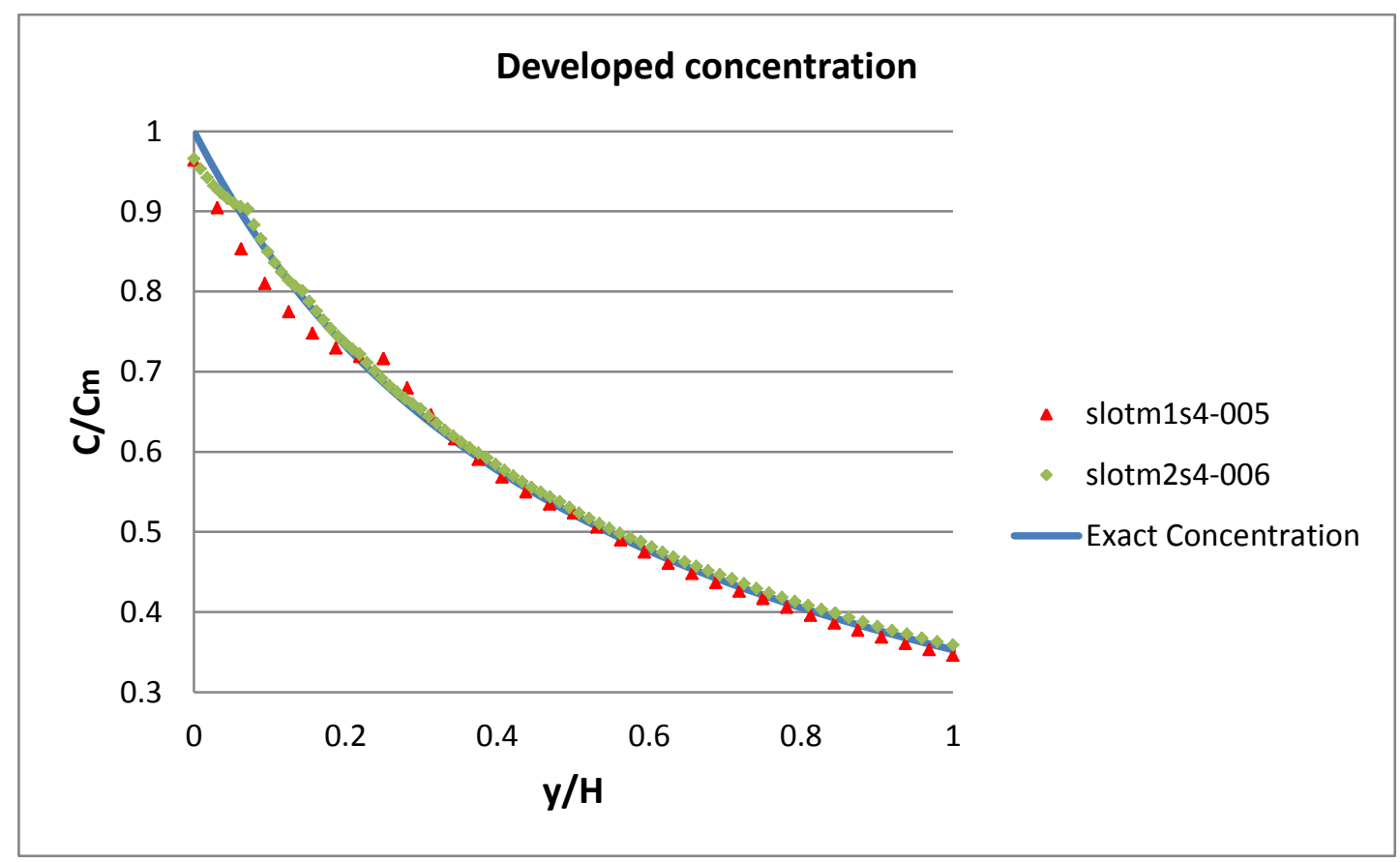

Figure 5.3: Developed concentration at the exit of the slot, for different meshes.

Figure 5.3 shows that the results for both meshes captures the same concentration values at the wall and at the centerline, and that in most part of the cross section the predictions have the same concentration values and slope of the exact solution. As expected, the accuracy is smaller near the centerline, where the solution is singular. Although mesh 1 shows a larger error in that region due to the lack of mesh refinement, we considered the solution to be accurate. The predictions of the full domain flow were obtained with elements having approximately the same size of elements from mesh 1 .

It can be stated that the mesh refinement needed is higher than for the case of Newtonian fluid flow with constant viscosity. This is because in the transport model, the gradients of several variables, like $\nabla \dot{\gamma}, \nabla c$ need to be evaluated; rather than its values alone.

\section{3.}

\section{General Features of Particle Concentration Field in Slot Coating Flow}

This section present the general features of particle concentration field in full domain slot coating flow. 
Figure 5.4 depicts the initial domain mesh. It is divided in 6 quadrangular regions. The number of elements per coordinate direction is presented in Table 5.3, giving 1,312 elements in total.

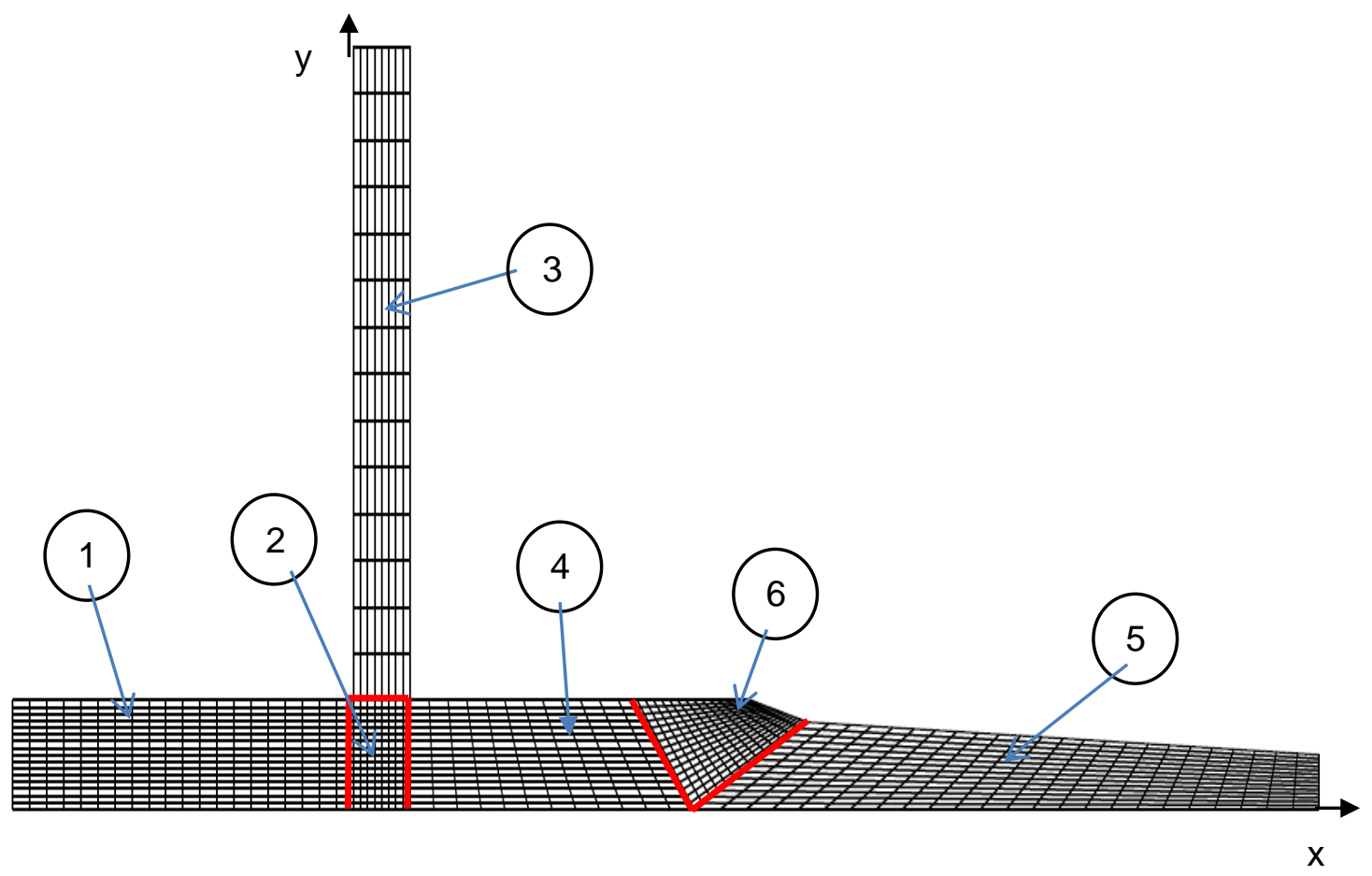

Figure 5.4: Domain mesh.

Table 5.3: Number of elements in the mesh.

\begin{tabular}{|c|c|c|c|}
\hline Region & y-direction & x-direction & Total per region \\
\hline 1 & 16 & 20 & 320 \\
\hline 2 & 16 & 8 & 128 \\
\hline 3 & 14 & 8 & 112 \\
\hline 4 & 16 & 12 & 192 \\
\hline 5 & 24 & 14 & 336 \\
\hline 6 & 16 & 14 & 224 \\
\hline
\end{tabular}

The parameters used as the base case for the analysis were:

- Capillary number:

$C a=0.1$

- Bulk viscosity:

$\eta=0.060 \mathrm{~Pa} . \mathrm{s}$

- Surface tension:

$\sigma=60 \mathrm{~g} / \mathrm{s}^{2}$

- Substrate velocity:

$V_{w}=100 \mathrm{~mm} / \mathrm{s}$

- Particle concentration:

$c=0.40$

- Particle radius:

$a=0.004 \mathrm{~mm}$

- Continuum phase viscosity:

$\eta_{0}=0.0119$ Pa.s 
- Coating gap:

$$
\begin{aligned}
& H_{0}=0.10 \mathrm{~mm} \\
& \text { - Coated layer thickness: } \quad t=0.05 \mathrm{~mm} \\
& \text { - Atmospheric pressure: } \quad P_{a t m}=0 \mathrm{~Pa}
\end{aligned}
$$$$
\text { - Vacuum pressure: } \quad P_{v a c}=-3700 \mathrm{~Pa}
$$$$
\text { - Particle migration constants: } \quad k_{c}=0.490, \quad k_{\eta}=0.735
$$

The capillary number is defined base on the bulk viscosity, i.e. the viscosity evaluated at the average particle concentration of the suspension. The flow field obtained by setting the transport parameter to zero, $k_{c}=k_{\eta}=0$, corresponds to the flow with constant particle concentration, as shown in Figure 5.5. The velocity field is that of a Newtonian liquid with viscosity equal to the bulk viscosity. As stated before, this solution is used as initial guess for the flow with particle migration.

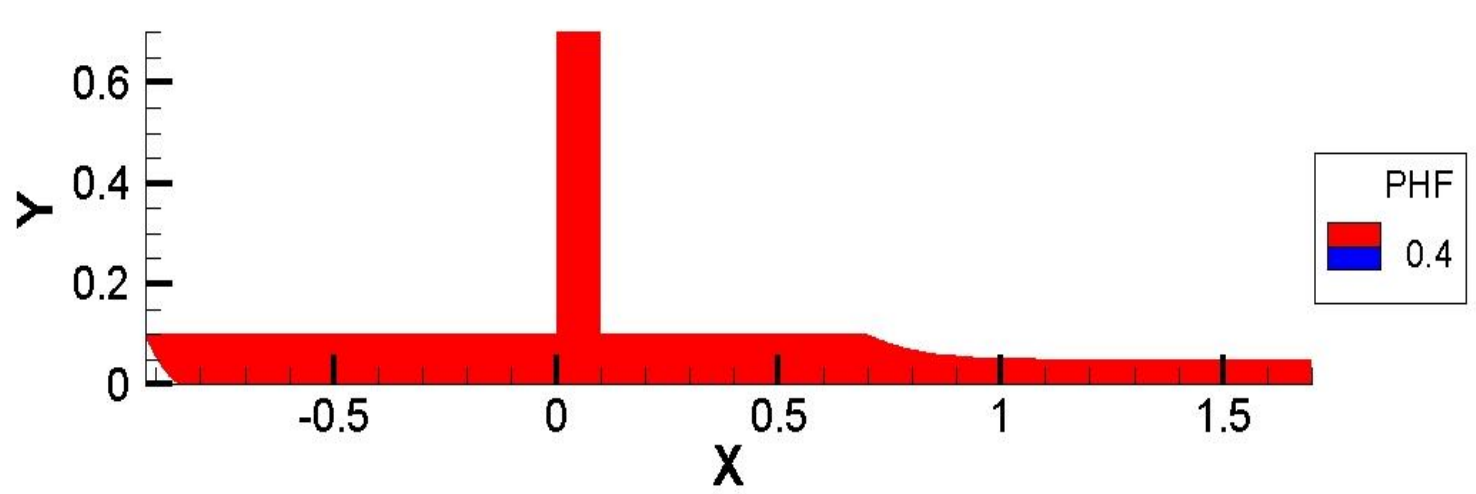

Figure 5.5: Slot coating flow of suspension without migration. Case "without.part.mig".

One of the main goals of the analysis is to study the effect of particle transport in the flow. Therefore, predictions of suspension flow considering particle transport are compared to predictions of Newtonian flow without particle migration, at which the viscosity is constant throughout the flow domain.

The upstream interface location results from the balance of viscous, capillary and pressure forces. The coordinate of the dynamic contact line of suspension flow without particle migration, at $C a=0.1$, is: $x_{d c l}=-0.85 \mathrm{~mm}$.

Figure 5.6 depicts concentration field of slot coating flow of suspension with particle migration at the conditions listed before. 


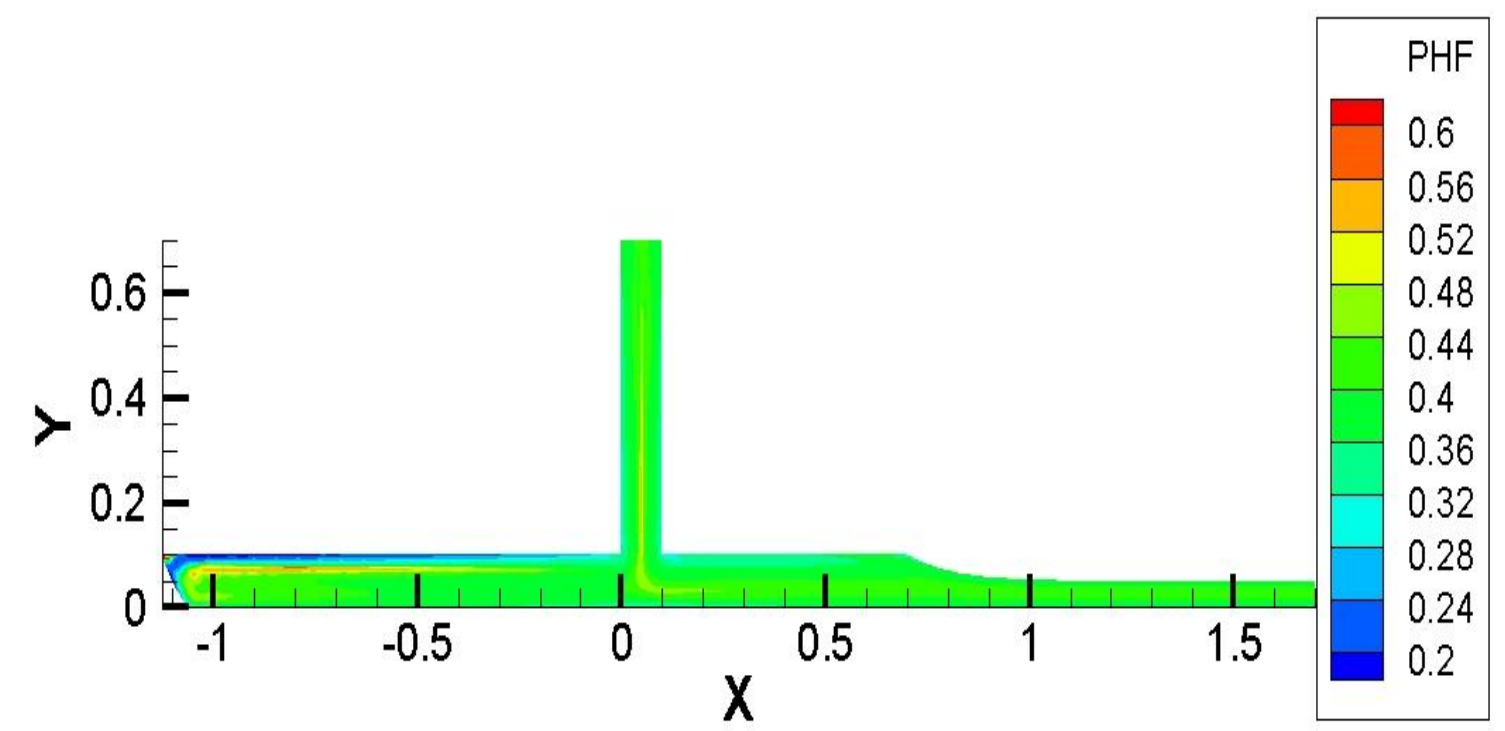

Figure 5.6: Slot coating flow of suspension with migration. Case "with.part.mig"

An analysis of the important observed features related to particle migration is presented for the different regions of slot coating flow.

\section{Feed slot region}

As analyzed before (section 5.2), the Poiseuille flow in the feed slot leads to particle migration towards the centerline, reaching maximum values there. The concentration field together with velocity vectors are plotted in Figure 5.7. The particles migrate toward the centerline; nevertheless maximum concentration is not reached at the end of the feed slot, because of particle diffusion transport towards the coating bead. It is remarked that this migration, at the end of the feed slot, is very high because in such a short distance, $\Delta y \approx 0.05 \mathrm{~mm}$, there is a sudden change in concentration along the streamline (high concentration gradient). 


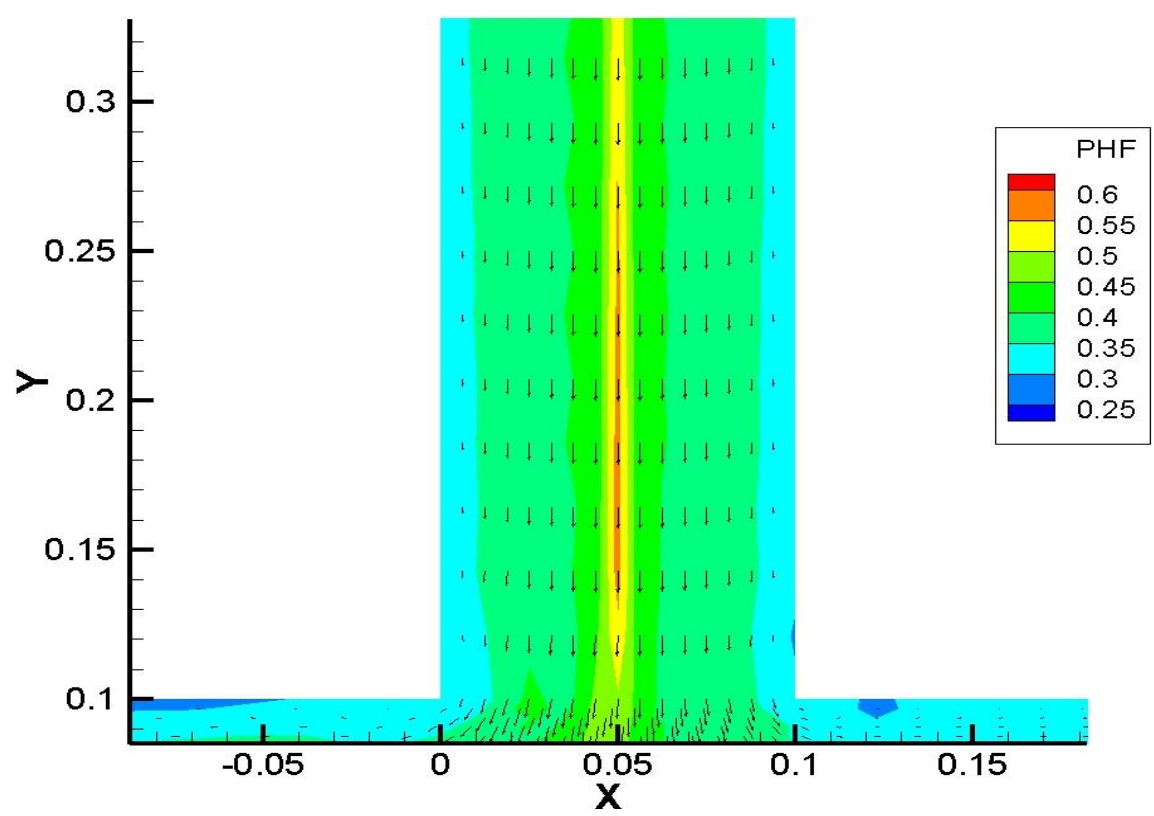

Figure 5.7: Feed slot region of case "with.part.mig".

\section{Upstream region}

The coordinate of the dynamic contact line of suspension with migration of particles $(C a=0.1)$ is: $x_{d c l}=-1.06 \mathrm{~mm}$. A large displacement (about $\sim 25 \%$ ) towards the upstream side is observed, when compared to the Newtonian flow, meaning that the pressure gradient is lower when particle migration occurs. This can be explained because the low viscosity (lower than bulk viscosity) near the slot die lip and substrate. The lower pressure gradient is clearly observed in Figure 5.8.

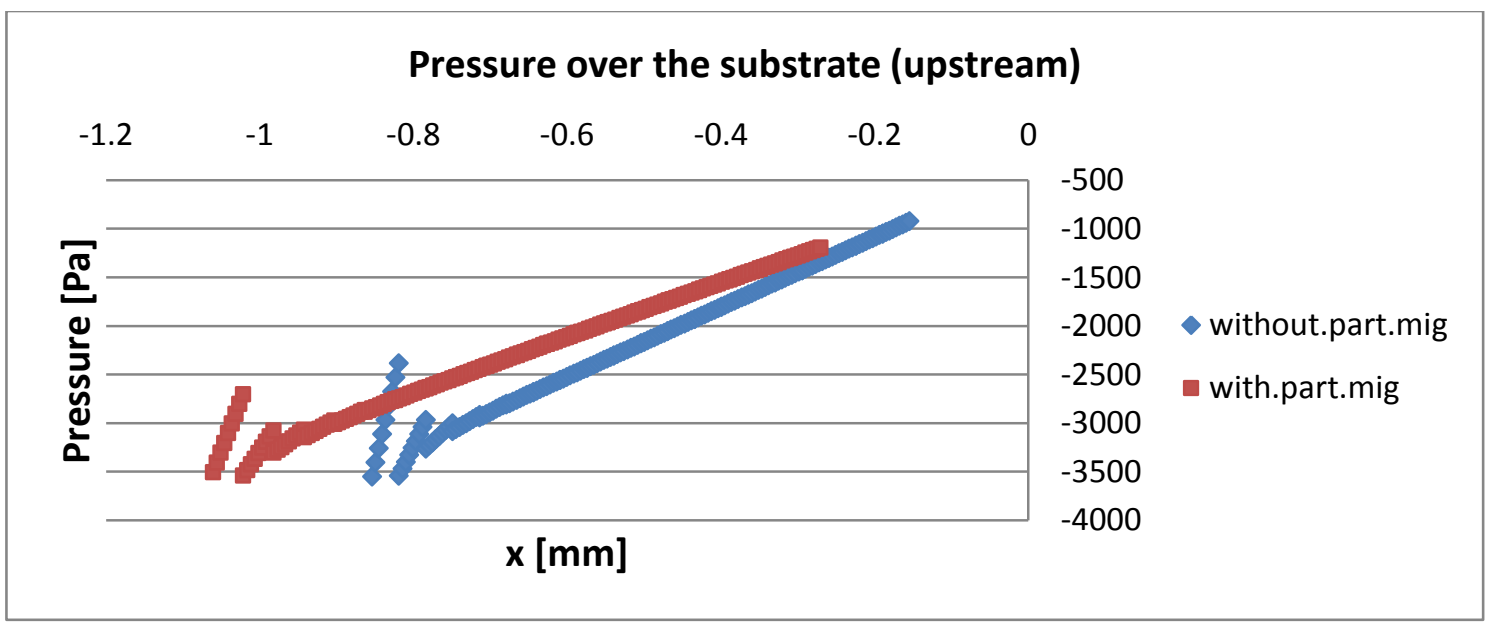

Figure 5.8: Comparison of the pressure over the substrate in the upstream region. 
The combination of Poiseuille (pressure-driven) and Couette (viscous drag) flow under the upstream die lip generates shear rate gradient that lead to strong particle migration, as shown in Figure 5.9. Local concentration, higher and lower than the bulk concentration $\left(c_{\text {bulk }}=0.4\right.$ ) is observed in different regions of the flow.

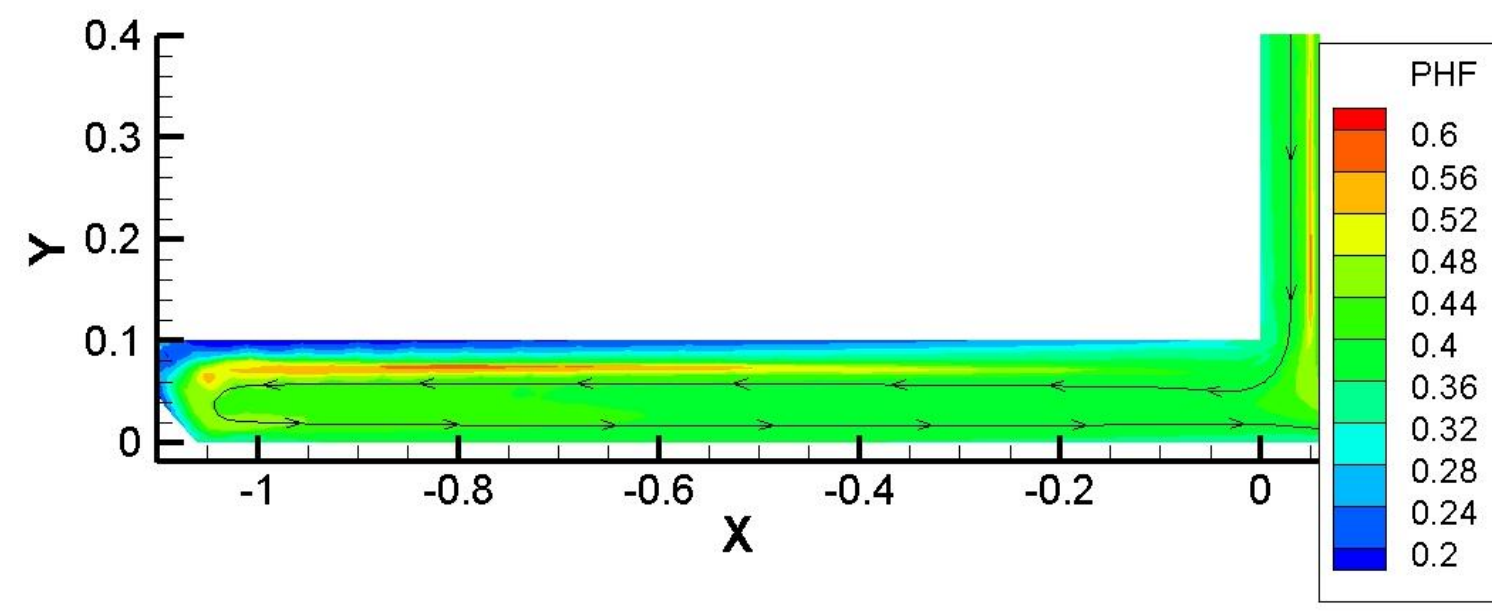

Figure 5.9: Upstream section of case "with.part.mig".

A magnified view of the flow near the upstream free surface (Figure 5.10) shows that regions of high concentration of particles are formed near the static contact line and in a layer of zero shear stress close to the die surface.

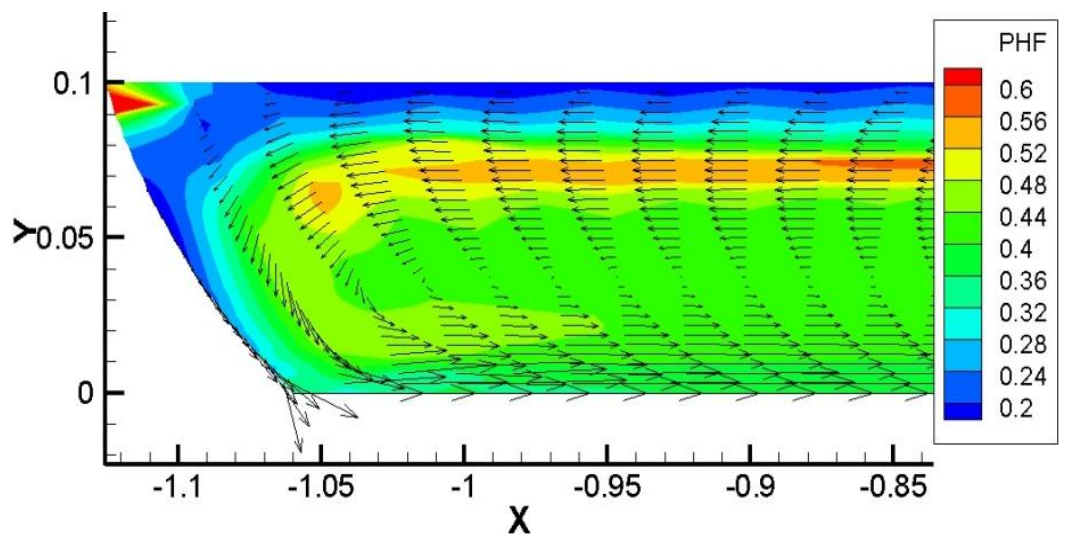

Figure 5.10: Magnified view of upstream flow. Case "with.part.mig".

The formation of a zone of maximum concentration at the upstream region depends on the length of the upstream coating bead. When the upstream zone is sufficiently long, the concentration profile reaches the maximum concentration 
(close to packing fraction) as was shown in Figure 5.9. An example of particle migration on a shorter upstream zone is presented in Figure 5.11. This result was obtained by changing the vacuum pressure. Particles migrate away from regions of high shear rate. However, because of the short upstream length, the particle concentration never reaches the close-packing concentration.

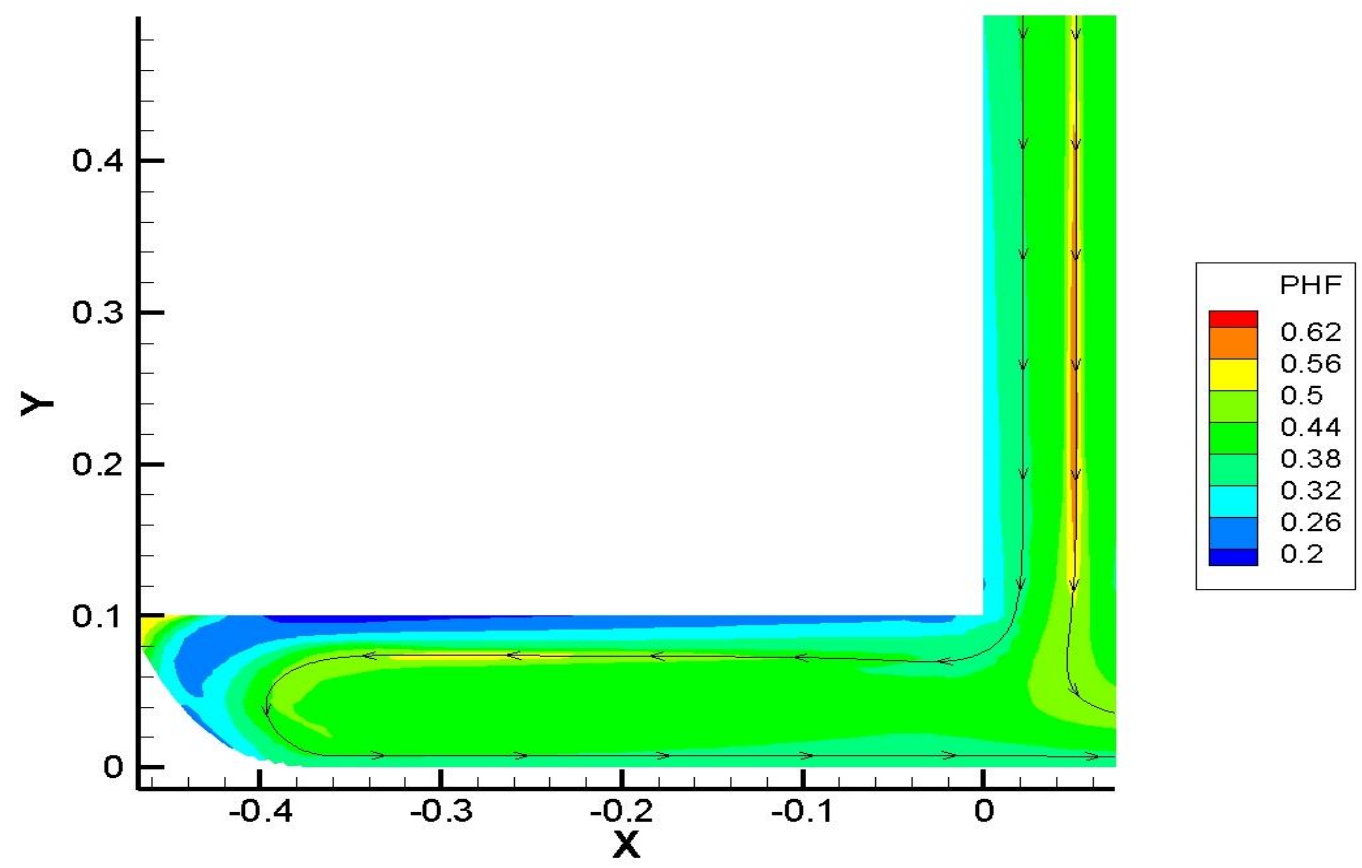

Figure 5.11: Shorter upstream region.

\section{Downstream region}

Figure 5.12 depicts the concentration distribution on the downstream region. Concentration scale was changed in order to visualize better this scalar field. It can be observed that the centerline of the feed slot, with higher concentration, is convected to the downstream region, leading to nonuniform distribution in the coated layer. 


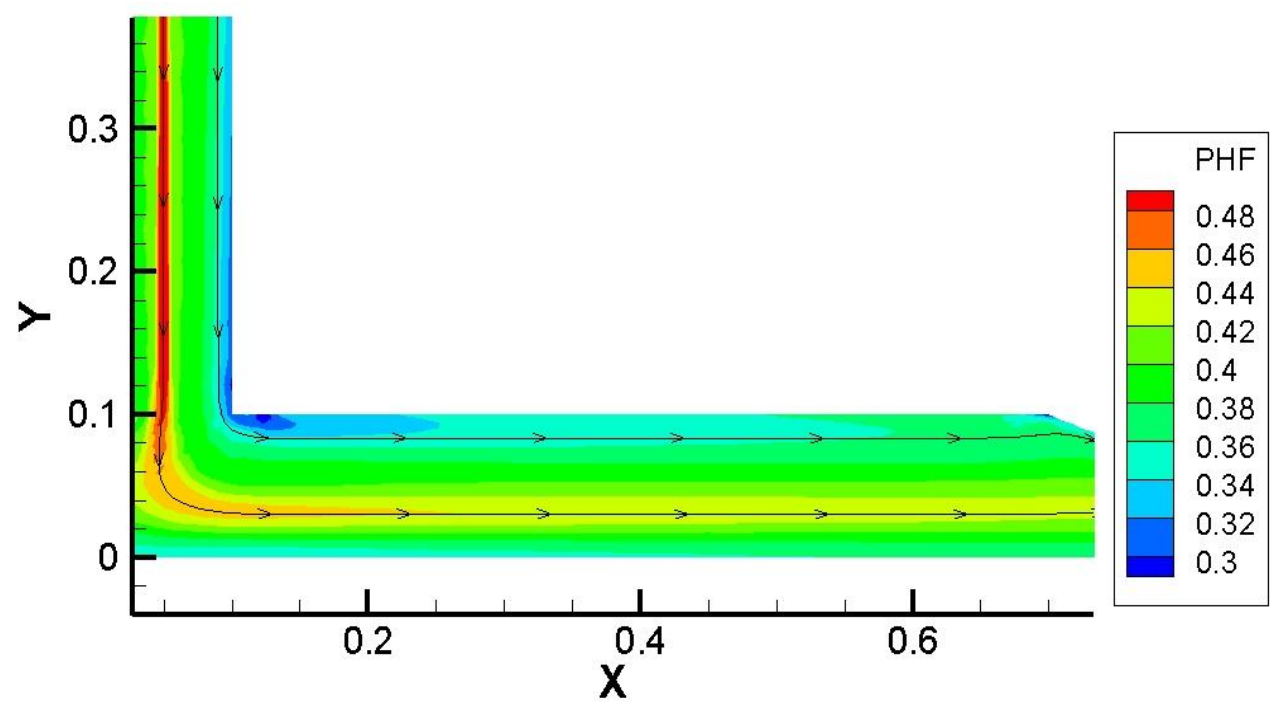

Figure 5.12: Concentration at the downstream region. Case "with.part.mig".

The flow of Newtonian fluid with constant viscosity (case "without.part.mig"), in the coating bead region, has a region between the interval $x \in[0.2 ; 0.65]$, where the pressure is constant. If the total flow rate is $q=V_{w} H_{0} / 2$ the flow in this region is well described by a simple Couette flow (Higgins \& Scriven, 1980); therefore, there is no pressure gradient (see Figure 5.13). On the other hand, the suspension flow shows a non-vanishing pressure gradient under the downstream die lip, as observed in Figure 5.13. An adverse pressure gradient is necessary to set an imposed flow rate.

A simplified model of the suspension flow can be made to have a better understanding of the kinematic in this region. Assuming a one-dimensional developed velocity and concentration field and viscosity as a function of concentration, the resulting $1 \mathrm{D}$ model that describes this situation is:

$$
\frac{d \tau_{y x}}{d y}=\frac{d p}{d x}
$$

where the shear stress is:

$$
\tau_{y x}=\eta(c) \dot{\gamma}_{y x}
$$

Then, substituting equation 5.2 into equation 5.1, results: 


$$
\eta(c) \frac{d^{2} u}{d y^{2}}+\frac{d \eta(c)}{d y} \frac{d u}{d y}=\frac{d p}{d x}
$$

Integration from the wall to an internal coordinate, the equation 5.3 leads to:

$$
u(y)=\frac{d p}{d x} \int_{0}^{y} \frac{y}{\eta} d y+\eta_{w} \dot{\gamma}_{w} \int_{0}^{y} \frac{1}{\eta} d y+V_{w}
$$

Equation 5.4 is the general equation to calculate the velocity profile in a fully developed flow that results from the combination of Couette and Poiseuille contributions. The equation 5.4 shows a nonlinear behavior of the velocity profile, even for the zero pressure gradient case, due to the viscosity dependence on concentration. Therefore, a non-constant shear stress, $\tau_{y x}$, is expected and the pressure gradient should be different from zero.

A plot of the velocity and concentration profile across a section at $x=0.40 \mathrm{~mm}$ is presented in Figure 5.14. The plot confirmed the nonlinear velocity profile due to stratified viscosity. It is important to observe that slightly higher gradients in velocity are observed near the substrate and slot die lip, because of the lower concentration near the walls and consequently lower liquid viscosity.

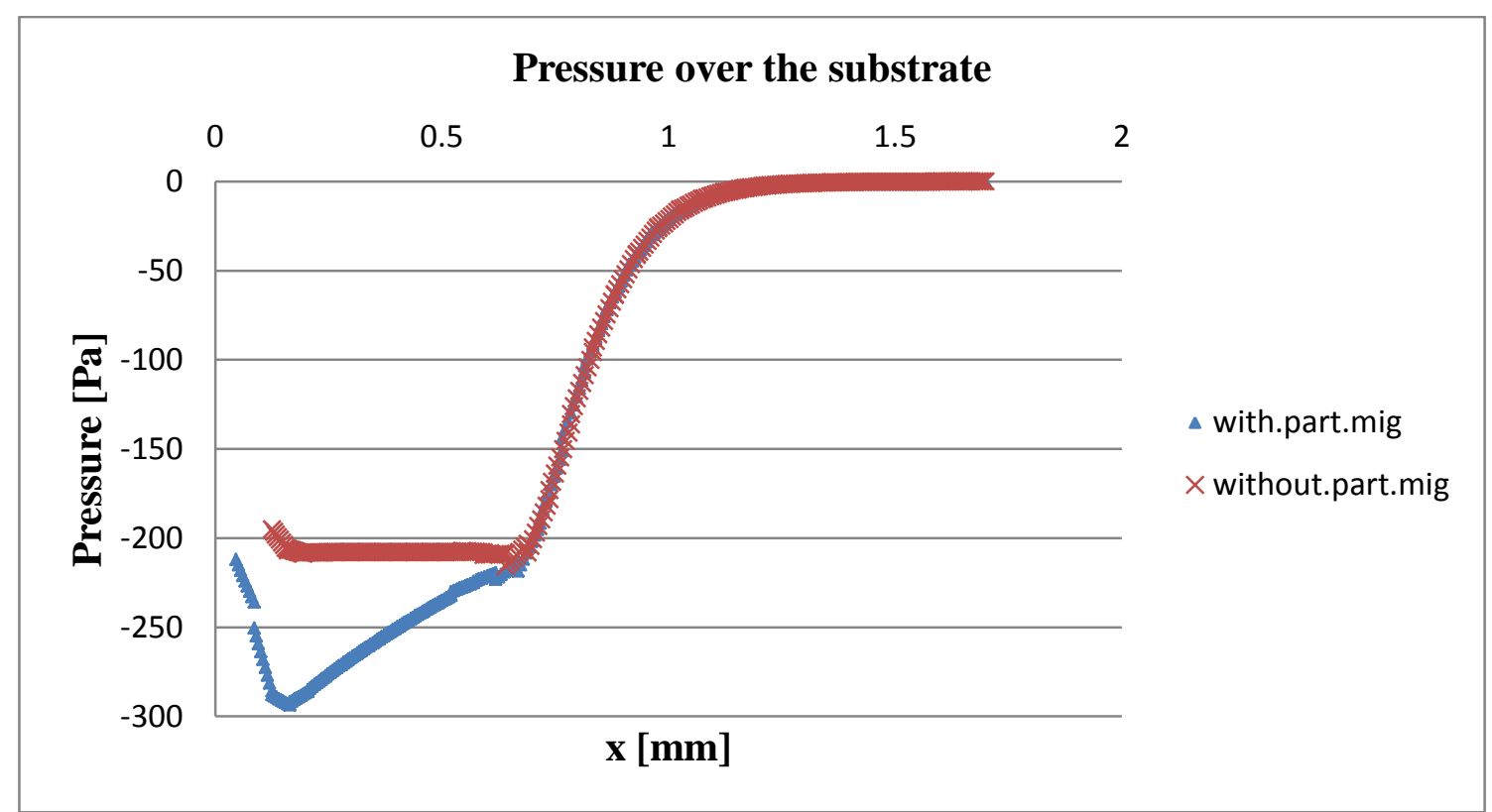

Figure 5.13: Pressure over the substrate in downstream region. 


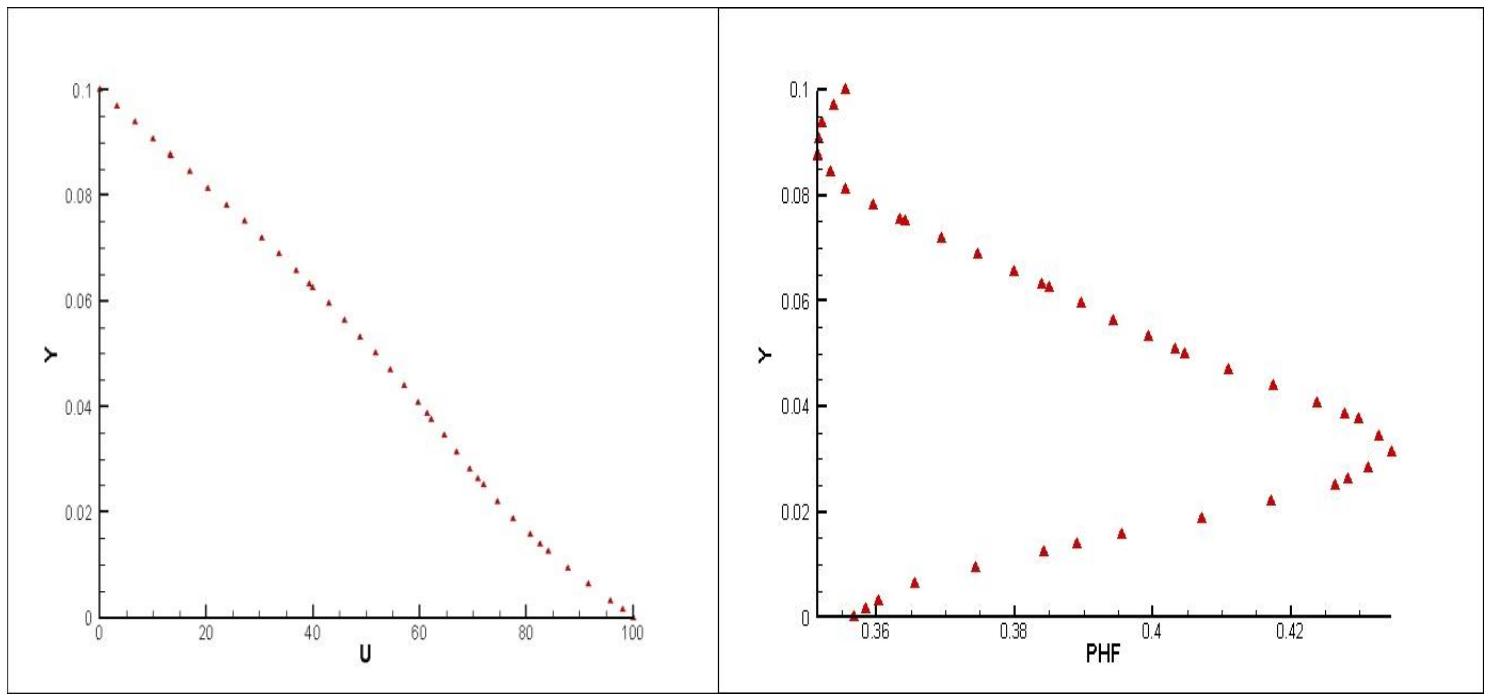

(a)

(b)

Figure 5.14: (a) Velocity and (b) concentration (PHF) profiles at $x=0.40 \mathrm{~mm}$. Case "with.part.mig"

In addition, it can be deduced from Figure 5.14 that the particle migrates along the downstream region due to viscosity gradient (proportional to $\sim \dot{\gamma} \nabla c$ ), because of the small shear-rate gradient. It is noted that the shear-rate is quasiconstant.

\section{Downstream interface}

The downstream interfaces configurations were identical for both cases analyzed. However, a gradient in particle distribution is found along the interface, as shown in Figure 5.15. The concentration is plotted versus the arc length, beginning from the static contact line to the end of the interface. The concentration never reaches the bulk concentration, even at the developed coated layer. In addition, an important feature is the high concentration gradient near the static contact line. 


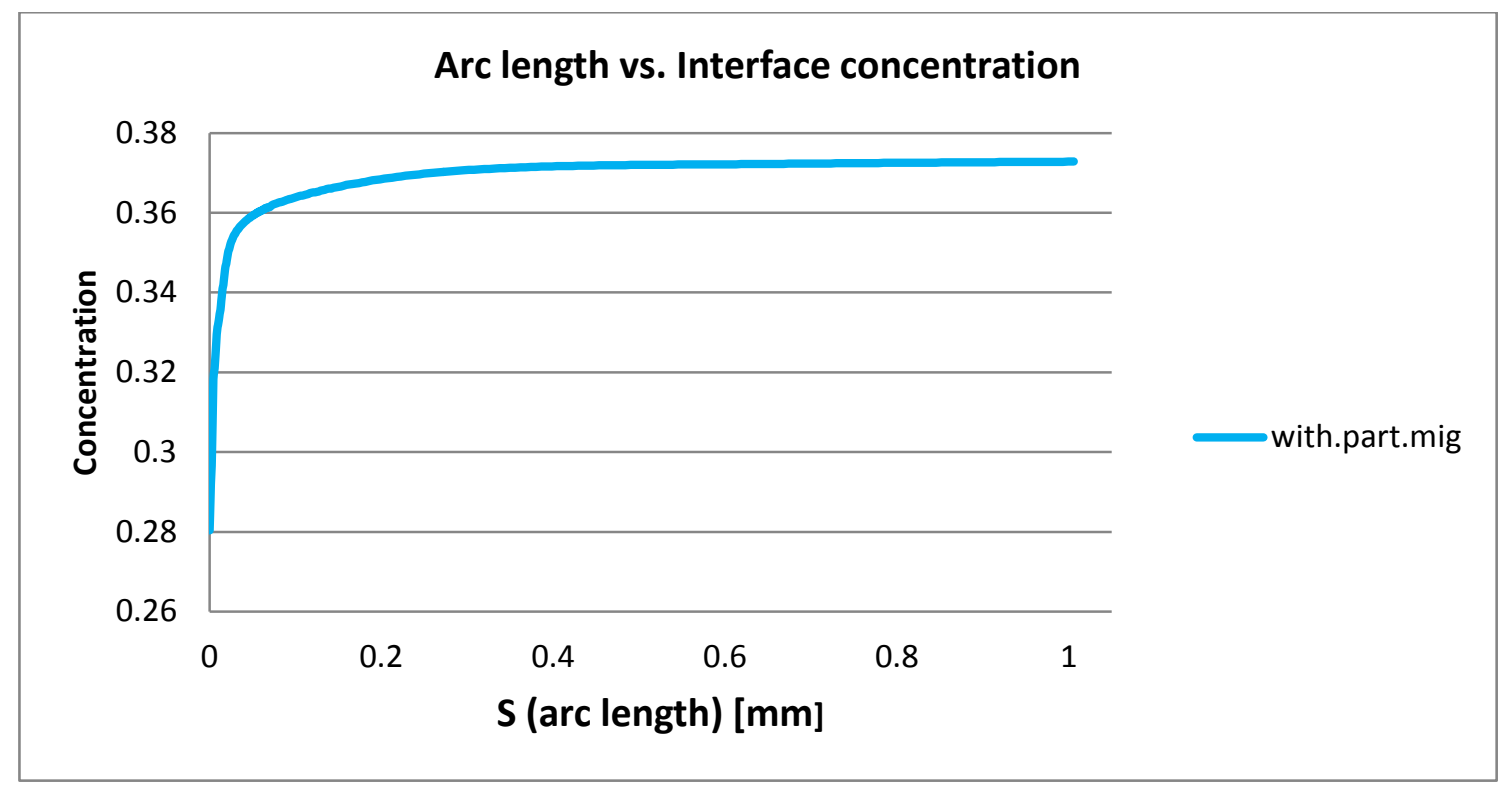

Figure 5.15: Concentration at the downstream interface along interface length.

\section{Developed coated layer}

This region corresponds to the deposited liquid over the substrate. It is important to know how particles are distributed over the substrate, in order to prepare further processes of drying and control desired properties of the final coated layer. Figure 5.16 depicts the developed suspension flow.
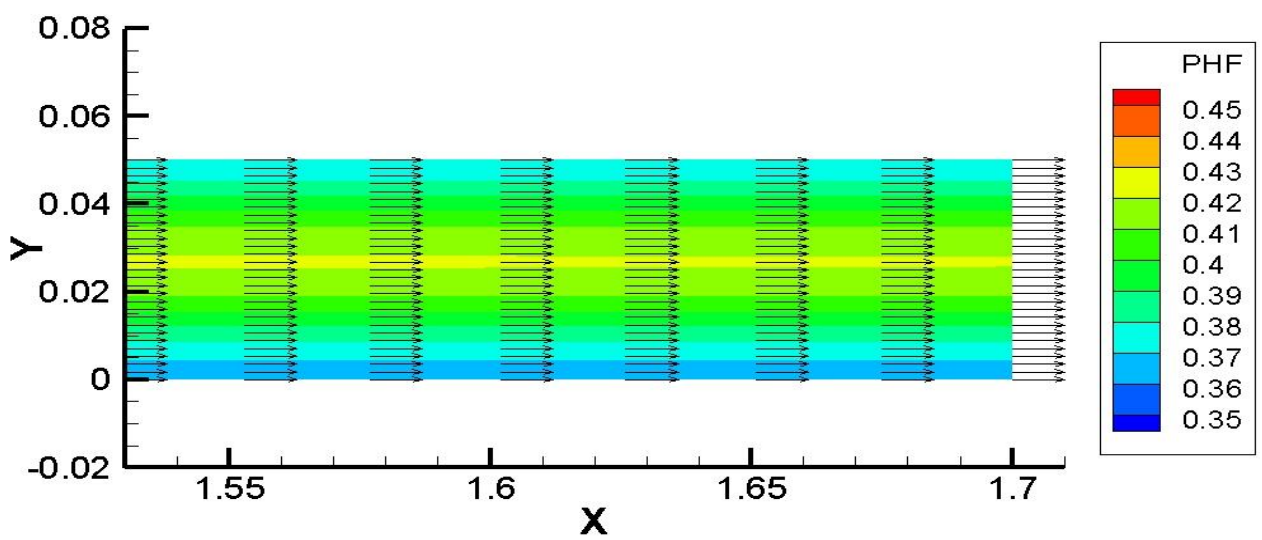

Figure 5.16: Concentration across the developed coated layer.

The coated layer has constant velocity profile, i.e. plug flow with vanishing shear rate. Hence, the migration mechanisms, considered in this research, that take into account shear-induced particle migration are negligible; therefore, the 
particles move over horizontal streamlines (do not migrate), and the concentration gradient does not change along the flow direction. It could be said that the suspension moves like a rigid body.

The particle concentration across the developed coated layer is plotted in Figure 5.17. A region of high concentration is observed at the center region. These were the particles convected from the centerline of the feed slot.

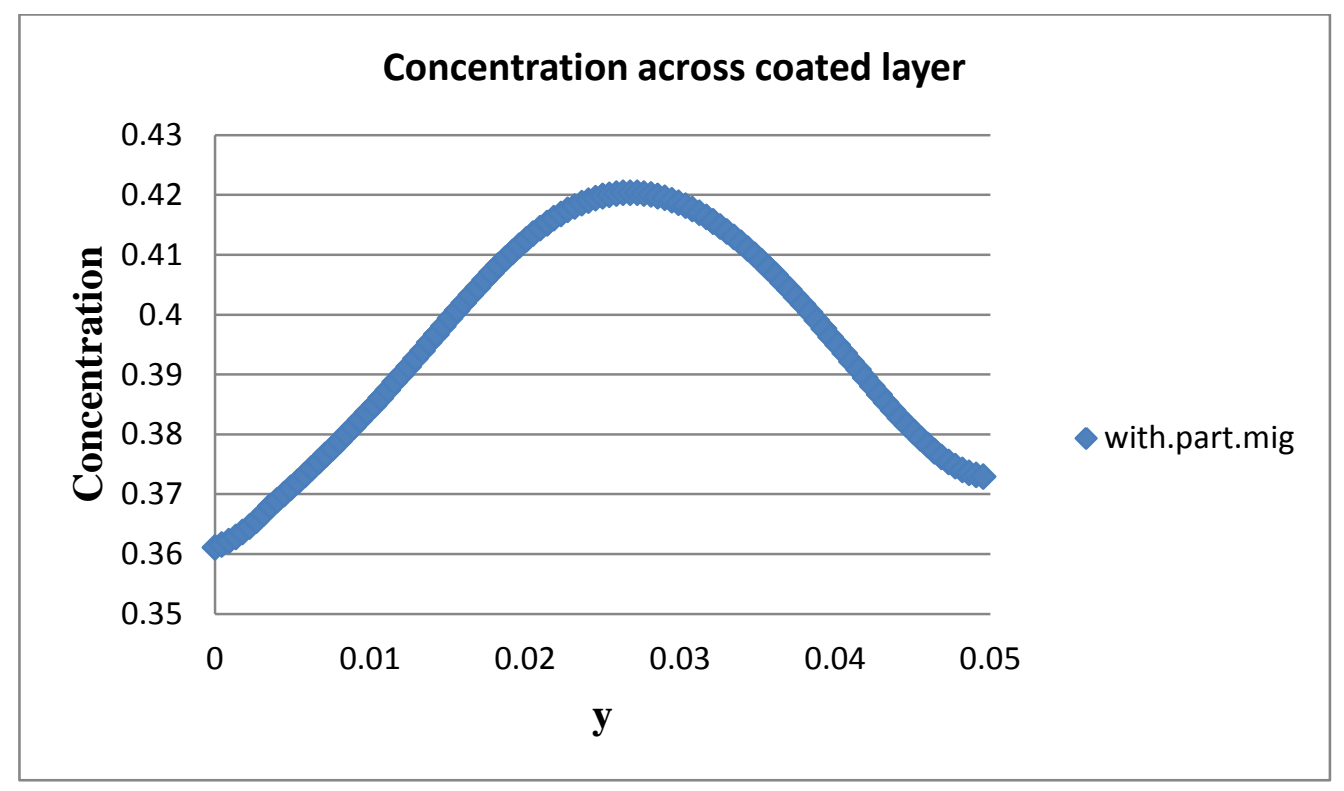

Figure 5.17: Particle concentration across coated layer.

\section{4 . Influence of Film Thickness on Particle Concentration}

The thickness of the coated layer can be lowered by reducing the flow rate. Assuming incompressible flow, mass conservation principle leads to flow rate being directly proportional to film thickness, keeping constant the substrate speed.

Several theoretical and experimental investigations (Carvalho \& Kheshgi, 2000; Lee et al., 2002; Romero et al., 2004) show that the flow pattern changes deeply as the film thickness varies. Therefore, there are expected different particle concentration distributions in the flow, as the film thickness falls.

According to the model for one-dimensional flow of a Newtonian liquid in the coating bead (Romero, 2003) the velocity profile resulting from a combination of Couette and Poiseuille flows is written as a function of the imposed flow rate $q$ and web speed as: 


$$
u(y)=\left(-6 \frac{q}{H_{0}^{3}}+3 \frac{V_{w}}{H_{0}^{2}}\right) y^{2}+\left(6 \frac{q}{H_{0}^{2}}-4 \frac{V_{w}}{H_{0}}\right) y+V_{w}
$$

At $t=\frac{H_{0}}{3}$ the shear-rate at the upstream wall is zero, $\dot{\gamma}_{x y}\left(y=H_{0}\right)=0$. This is the flow rate or film thickness $\left(t<\frac{H_{0}}{3}\right)$ at which a recirculation appears under the downstream die lip.

As it was pointed out in equations 5.1 to 5.4 , the velocity and shear-rate profiles for a suspension are different than that of a Newtonian liquid with constant viscosity. Therefore, the point where the backflow begins would be different. It is expected to be slightly different from the Newtonian liquid, because as it could be observed in Figure 5.14 the velocity profile is slightly nonlinear.

It is important to analyze the case at the onset of the recirculation under the downstream die lip. The zero shear rate gradient zones occur at the die wall; thus, particle migration towards this region is expected, reaching maximum concentration values. This can have a strong effect on the interface shapes, and concentration at the downstream interface and coated layer.

If the coated layer thickness is less than $1 / 3$ of the coating gap, a recirculation is present under the downstream slot die lip. It is important to know the particle distribution in this region and how the recirculation affects the concentration field. In addition, as in the previous case, it is crucial to observe the influence of the flow pattern in the final downstream interface shape, and particle distribution at downstream interface and coated layer.

The region of interest to be analyzed is the flow under the downstream die lip. Simulations were performed taking vacuum pressure values sufficiently high to keep the upstream interface far from the feed slot, so thin films could be obtained. A discussion on particle distribution on downstream free surface and final coated layer is presented next.

\subsection{1.}

\section{Analysis for $t=0.5 \mathrm{H}_{0}$}

The analysis for film thickness equal to half of the coating gap was performed at two capillary numbers. The parameters used for each simulation are shown in Table 5.4. Both cases have the same parameters except for substrate 
speed (capillary number). The goal is to observe how the viscous forces affects particle distribution and interface shape as they become stronger relative to capillary forces.

Table 5.4: Parameters for slot coating simulation for $t=0.5 H_{0}$.

\begin{tabular}{|c|c|c|}
\hline Case & slots4-017 & slots4-015 \\
\hline Bulk viscosity & $0.060 \mathrm{~Pa} . \mathrm{s}$ & $0.060 \mathrm{~Pa} . \mathrm{s}$ \\
\hline Substrate velocity & $100.0 \mathrm{~mm} / \mathrm{s}$ & $1000.0 \mathrm{~mm} / \mathrm{s}$ \\
\hline Surface tension & $0.060 \mathrm{~kg} / \mathrm{s} 2$ & $0.060 \mathrm{~kg} / \mathrm{s} 2$ \\
\hline Capillary number & $\mathbf{0 . 1 0}$ & $\mathbf{1 . 0}$ \\
\hline Continuous phase viscosity & $0.0119 \mathrm{~Pa} . \mathrm{S}$ & $0.0119 \mathrm{~Pa} . \mathrm{s}$ \\
\hline Particle concentration & 0.40 & $4.0 \mu \mathrm{m}$ \\
\hline Particle radius & $4.0 \mu \mathrm{m}$ & $0.10 \mathrm{~mm}$ \\
\hline Gap size of slot $\left(H_{S}\right)$ & $0.10 \mathrm{~mm}$ & $0.10 \mathrm{~mm}$ \\
\hline Gap size of coating bead $\left(H_{0}\right)$ & $0.10 \mathrm{~mm}$ & $0.05 \mathrm{~mm}$ \\
\hline Film thickness $(\mathrm{t})$ & $0.05 \mathrm{~mm}$ & 1.20 \\
\hline$k_{c}$ & 1.20 & 1.80 \\
\hline$k_{\eta}$ & 1.80 &
\end{tabular}

The migration coefficients $\left(k_{c}\right.$ and $\left.k_{\eta}\right)$ were kept the same for all cases. Therefore, we are assuming that the migration coefficients are a suspension characteristic that measure migration intensity and that they are independent of flow rate.

\section{Coating bead}

Figure 5.18 depicts the two-dimensional concentration distribution in the downstream region for both cases. It can be observed that the high concentration layer located near the centerline of the feed slot is convected to the downstream region and final coated layer. 

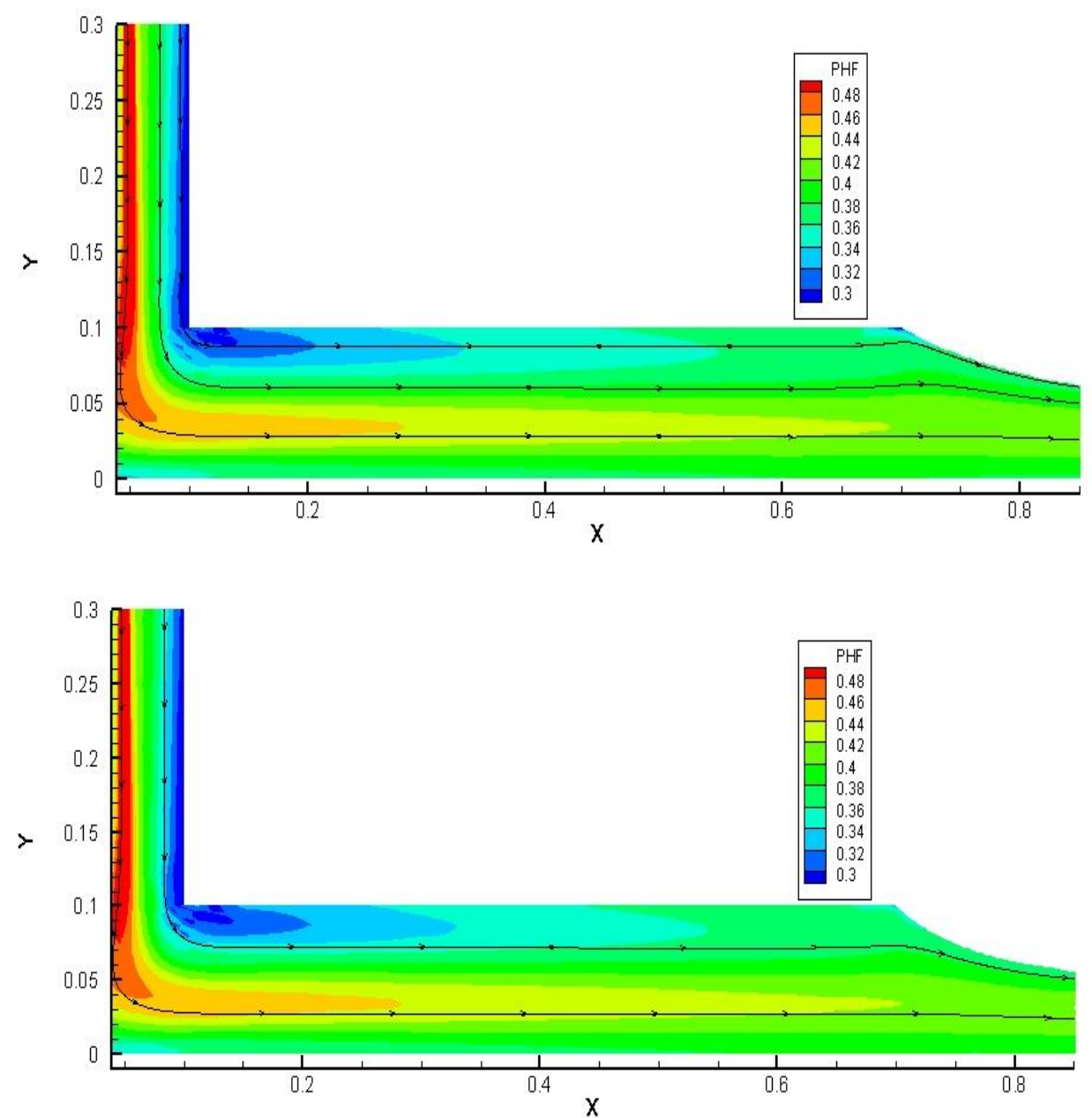

(b)

Figure 5.18: Two-dimensional particle distribution at coating bead for (a) $\mathrm{Ca}=0.10$, "slots4-017" and (b) $C a=1.0$, "slots4-015".

Migration mechanisms in the flow under the downstream die lip tend to homogenize the concentration distribution. According to the diffusive flux model, the main driving migration mechanism must be due to concentration gradient, because for this case, the shear-rate value is nearly constant. It can be observed in Figure 5.18 that the particle distribution for both cases is practically the same, even when there is a difference in shear-rate of about 10 times.

A plot of velocity profile at coordinate $x=0.40 \mathrm{~mm}$ confirms the affirmation made for shear-rate, as seen in Figure 5.19. Both profiles are compared with their respective Newtonian case, which is linear. 


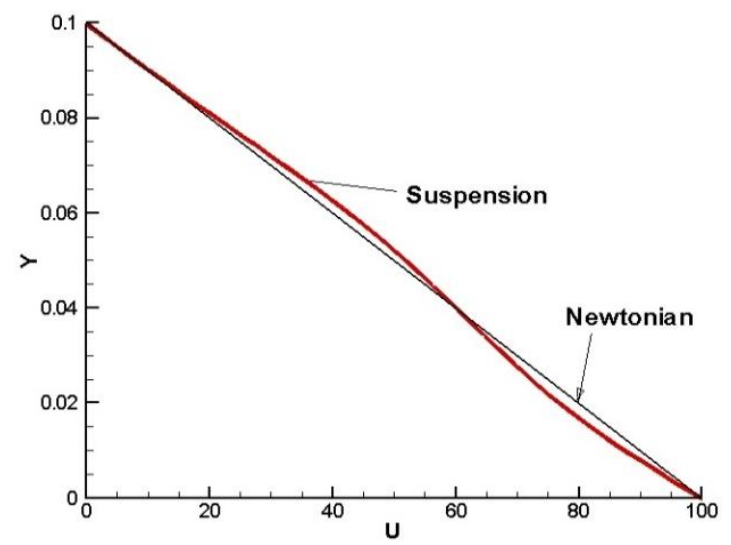

(a)

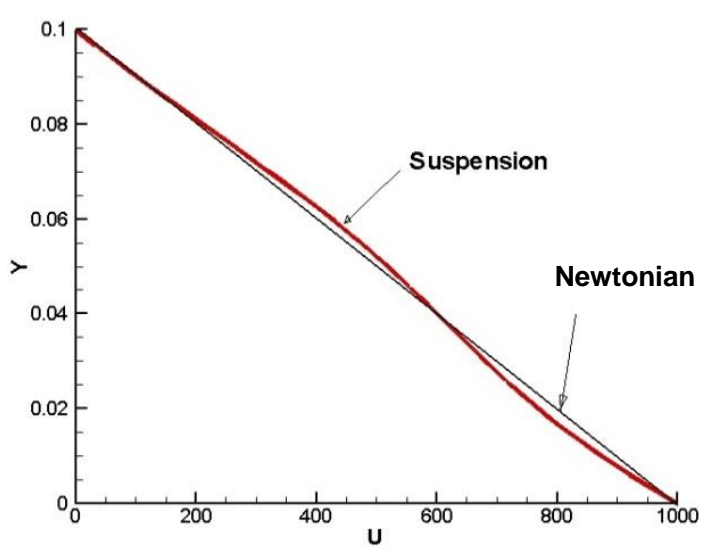

(b)

Figure 5.19: Velocity profile at $x=0.40 \mathrm{~mm}$ for (a) $\mathrm{Ca}=0.10$, "slots4-017" and (b) $C a=1.0$, "slots4-015".

The discrepancy between suspension and Newtonian liquid velocity profile arises due to viscosity stratification, and consequently concentration gradient at that cross section. Then concentration profile, plotted at the same coordinate $(x=0.40 \mathrm{~mm})$, is presented in Figure 5.20.

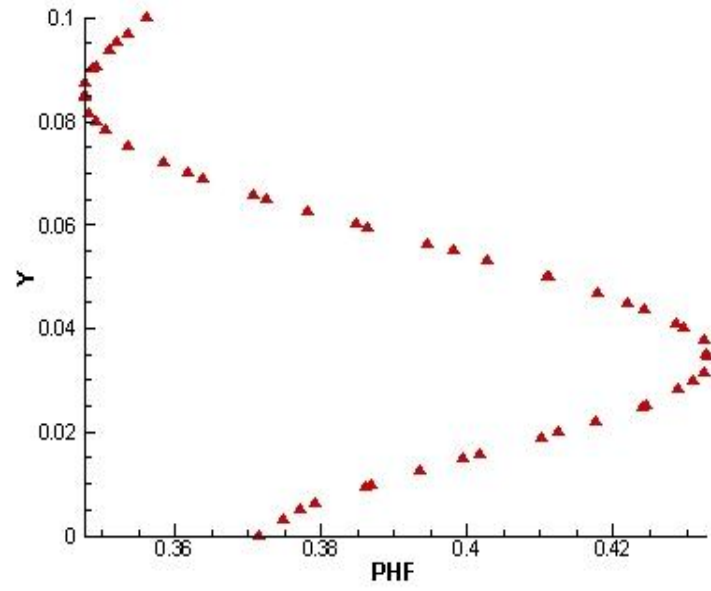

(a)

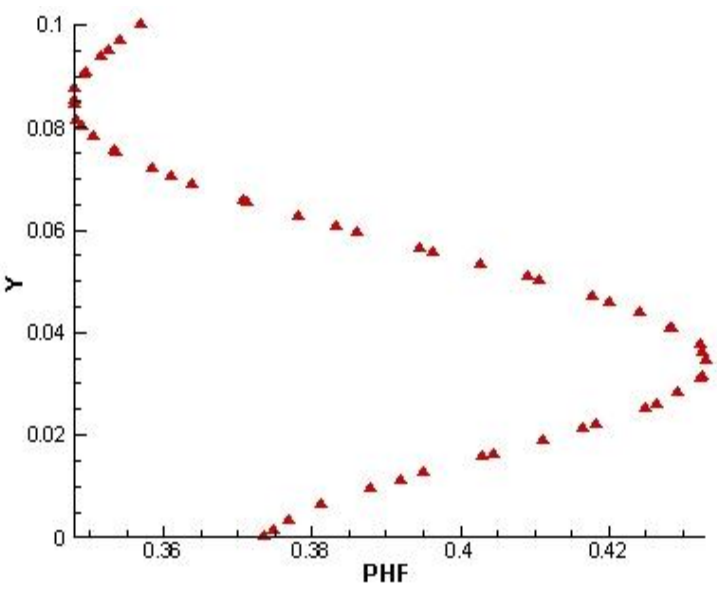

(b)

Figure 5.20: Concentration profiles at $x=0.40 \mathrm{~mm}$, for (a) $\mathrm{Ca}=0.10$, "slots4-017" and (b) $\mathrm{Ca}=1.0$, "slots4-015".

A layer of high particle concentration is formed at $y=0.04 \mathrm{~mm}$. Near the substrate and die surface the particle concentration is below the bulk value $\left(c_{\text {bulk }}=0.4\right)$. 
Finally, a pressure gradient over the substrate occurs due to variation in viscosity, and then in shear-stress (equations 5.1 and 5.2). The pressure over the substrate for suspension and Newtonian flow for each capillary number is plotted in Figure 5.21 and Figure 5.22. A discrepancy is observed due to zero pressure gradient for the case of Newtonian flow at coating bead.

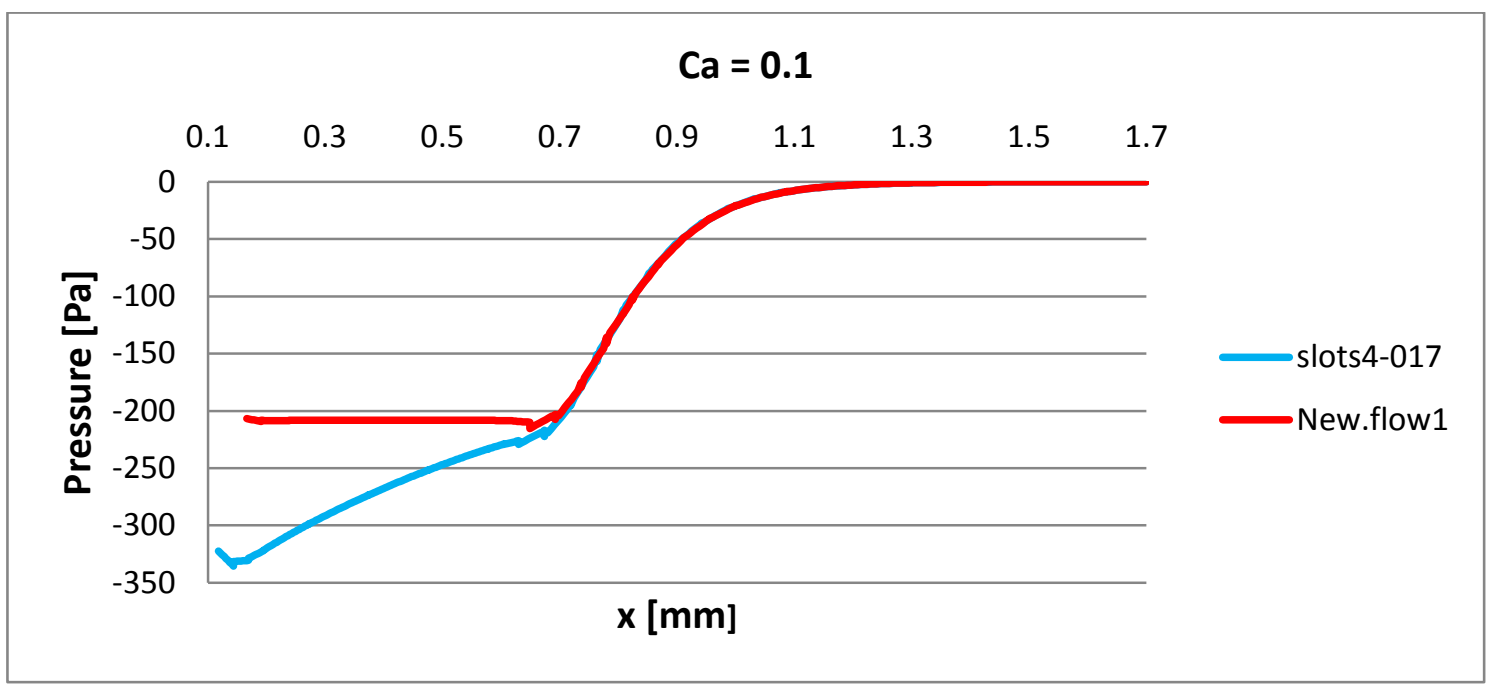

Figure 5.21: Pressure over substrate for $C a=0.10$.

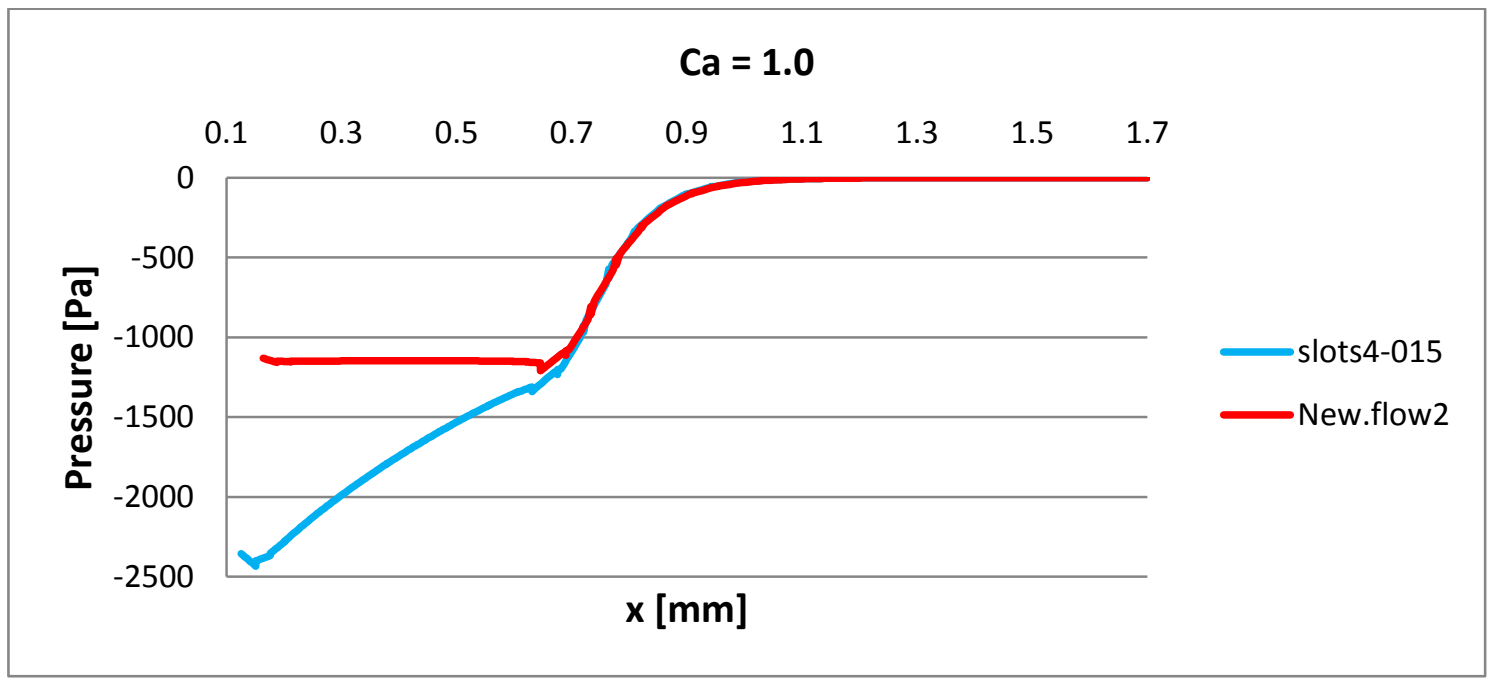

Figure 5.22: Pressure over substrate for $C a=1.0$.

\section{Free surface}

The concentration field near the downstream free surface is shown in Figure 5.23. The high particle concentration layer formed under the die lip is convected 
to the final deposited liquid layer. A region of low concentration is formed near the static contact line, since it is a region of high deformation rate. Along the free surface, the shear rate vanishes. Therefore, a strong particle concentration gradient is formed in the film formation region.

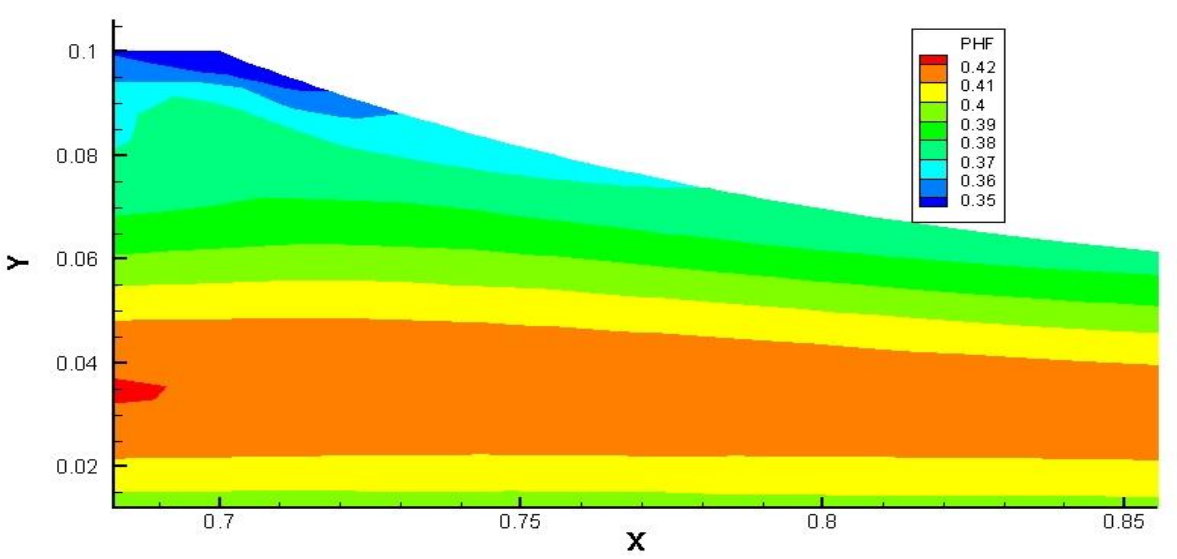

(a)

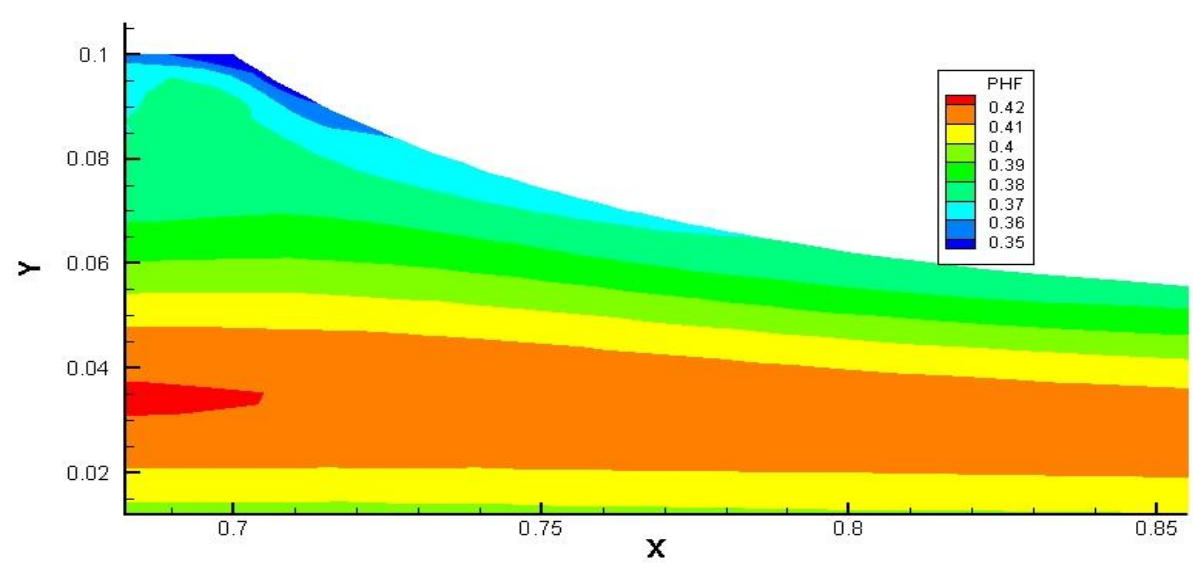

(b)

Figure 5.23: Concentration at free surface region for (a) $C a=0.10$, "slots4-017" and (b) $C a=1.0$, "slots4-015".

Figure 5.24 shows the comparison of the free surfaces of suspension and Newtonian flow, at $C a=1.0$. When particle transport is included, the interface is more curved. In the set of parameters analyzed here, this effect is weak. However, this change is curvature may indicate that the critical conditions of the low-flow limit, which is directly associated to the downstream interface, may be affected by the particle migration in the coating bead. No appreciable deviation was observed at $C a=0.10$. 


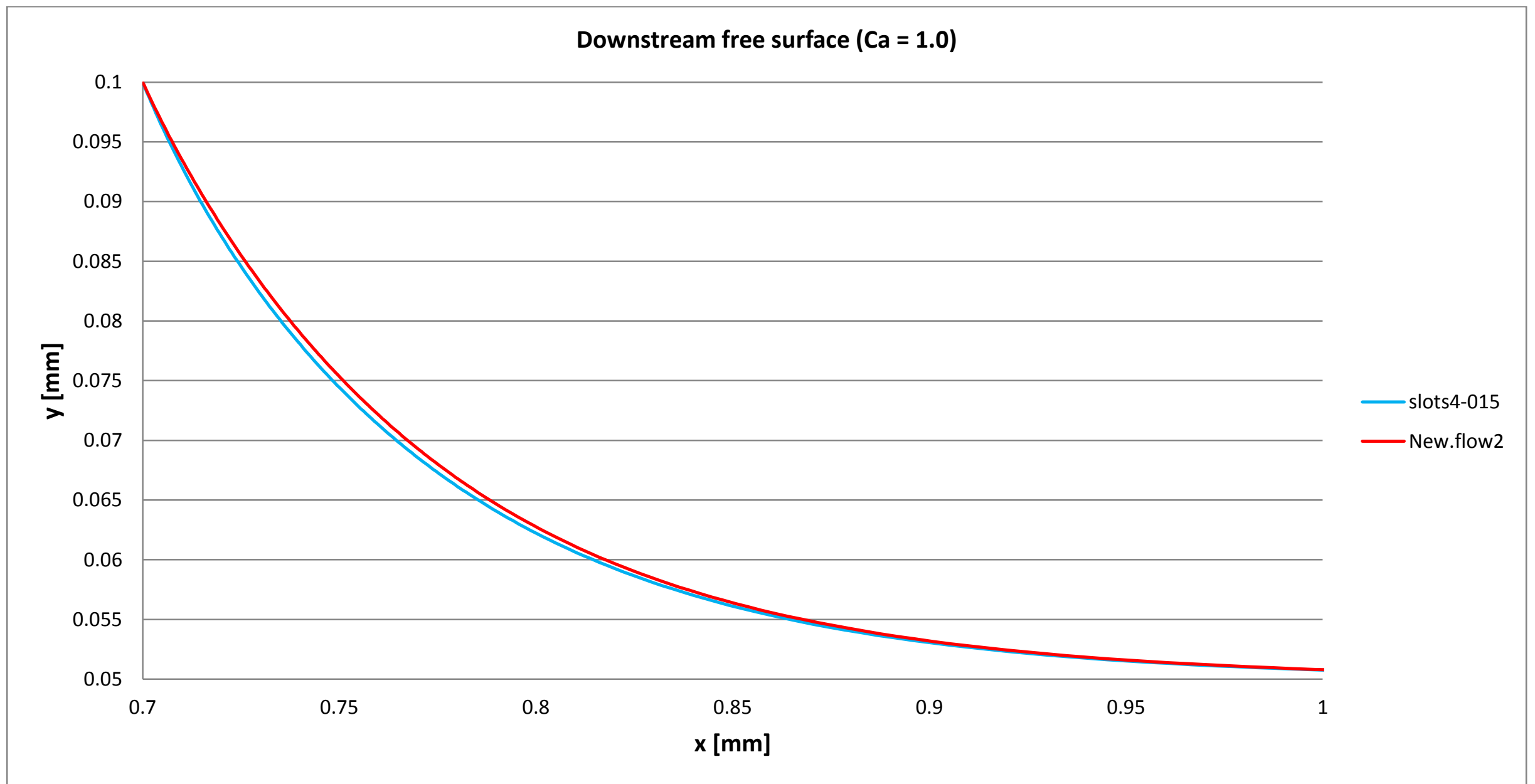

Figure 5.24: Free surface shape for Newtonian liquid ("New.flow2") and suspension ("slots4-015"), for Ca=1.0. 
The concentration along the interface is presented in Figure 5.25, starting from the static contact line to the developed coated layer. A high gradient in concentration near the static contact line is clearly observed. If surface tension dependence on particle concentration is included, this high gradient leads to strong Marangoni effect. It is also important to notice that the concentration on the top of the coated film changes with the capillary number.

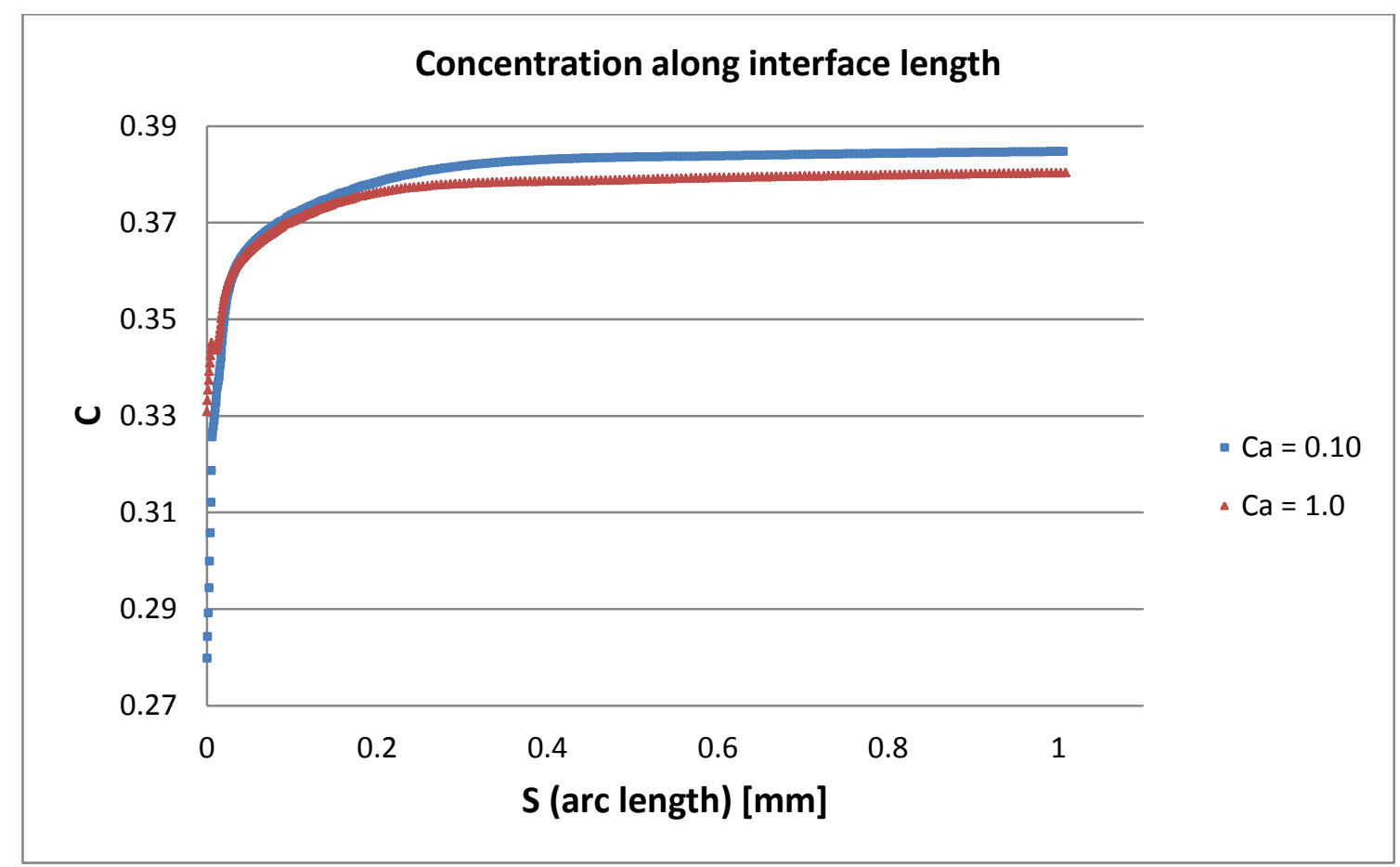

Figure 5.25: Concentration along the interface length for $\mathrm{Ca}=0.10$ ("slots4-017") and $C a=1.0$ ("slots4-015").

\section{Developed coated layer}

As it was explained it section 5.3, the particles in this region do not migrate due to developed flow state, then zero shear-rate gradient. A comparison of particle distribution across this layer is shown in Figure 5.26. Particle concentration distribution presents a region of high concentration near the center of the film, which was convected from feed slot centerline. It is important to note the concentration at the free surface is slightly higher for suspension flow with lower capillary number. The non-uniform concentration profile may have important implications on the drying process. 


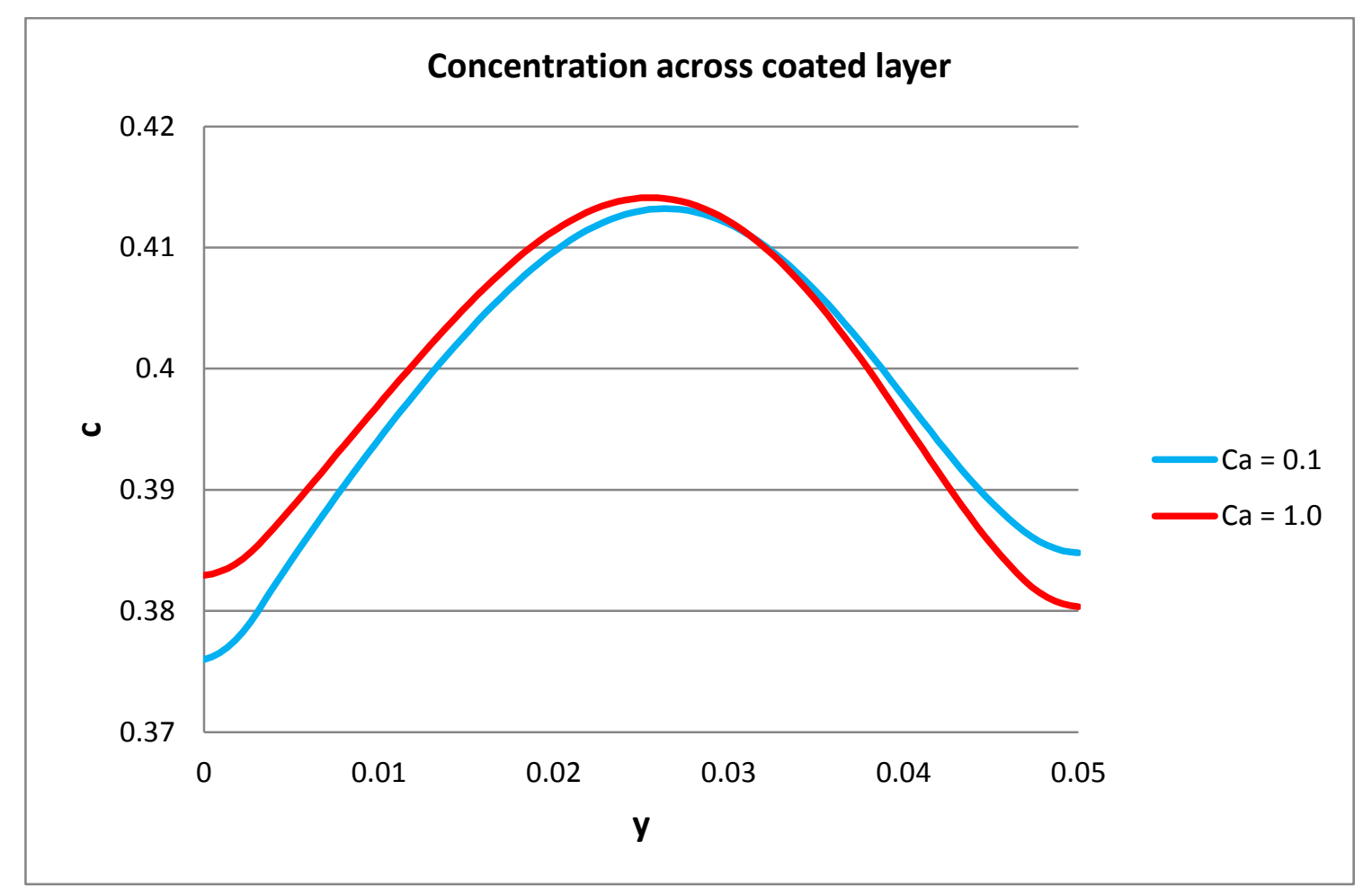

Figure 5.26: Particle concentration across coated layer for $\mathrm{Ca}=0.10$ ("slots4-017") and $C a=1.0$ ("slots4-015").

\subsection{2.}

\section{Analysis when Recirculation Flow Starts}

As discussed before (equation 5.5), at $t=H_{0} / 3$, shear-rate vanishes at the downstream slot die lip. This is a situation interesting to analyze because, according to the diffusive flux model, particles will migrate towards the die wall, leading to higher concentration.

It was performed simulations for different film thickness close to $t=H_{0} / 3$. Convergence problems appear due to an increment in particle concentration above physical values (close packing concentration) near the downstream slot die lip. Equation 4.1 does not avoid concentration increment due to zero local velocity; therefore non-local shear-rate remains zero. Despite of convergence problems, it was possible to obtained results for two different capillary numbers at which accumulation of particles at slot die wall was observed. Table 5.5 presents the parameters used in each case reported here. 
Table 5.5: Parameters for slot coating simulation when recirculation flow starts.

\begin{tabular}{|c|c|c|}
\hline Case & slots4-038 & slots4-060 \\
\hline Bulk viscosity & $0.060 \mathrm{~Pa} . \mathrm{s}$ & $0.060 \mathrm{~Pa} . \mathrm{s}$ \\
\hline Substrate velocity & $100.0 \mathrm{~mm} / \mathrm{s}$ & $1000.0 \mathrm{~mm} / \mathrm{s}$ \\
\hline Surface tension & $0.060 \mathrm{~kg} / \mathrm{s} 2$ & $0.060 \mathrm{~kg} / \mathrm{s} 2$ \\
\hline Capillary number & $\mathbf{0 . 1 0}$ & $\mathbf{1 . 0}$ \\
\hline Continuous phase viscosity & $0.0119 \mathrm{~Pa} . \mathrm{s}$ & $0.0119 \mathrm{~Pa} . \mathrm{s}$ \\
\hline Particle concentration & 0.40 & 0.40 \\
\hline Particle radius & $4.0 \mu \mathrm{m}$ & $0.10 \mathrm{~mm}$ \\
\hline Gap size of slot $\left(H_{S}\right)$ & $0.10 \mathrm{~mm}$ & $0.10 \mathrm{~mm}$ \\
\hline Gap size of coating bead $\left(H_{0}\right)$ & $0.10 \mathrm{~mm}$ & $0.037 \mathrm{~mm}$ \\
\hline Film thickness $(\mathrm{t})$ & $0.037 \mathrm{~mm}$ & 2.0 \\
\hline$k_{c}$ & 2.0 & 3.0 \\
\hline$k_{\eta}$ & 3.0 &
\end{tabular}

\section{Coating bead}

Figure 5.27 depicts the two-dimensional concentration distribution under the downstream die lip for both cases analyzed. The particle distribution is virtually the same for both cases. The accumulation of particles along the die lip wall is clearly observed. The migration of particles towards the wall eliminates the high concentration layer observed in case $t=H_{0} / 2$. 


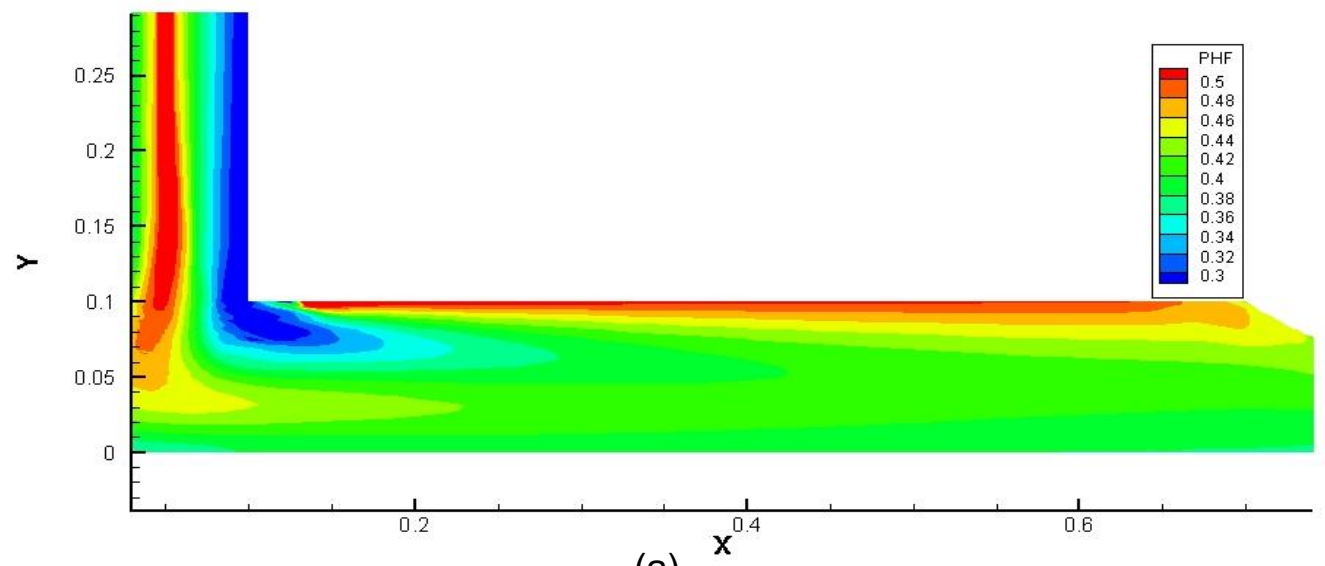

(a)

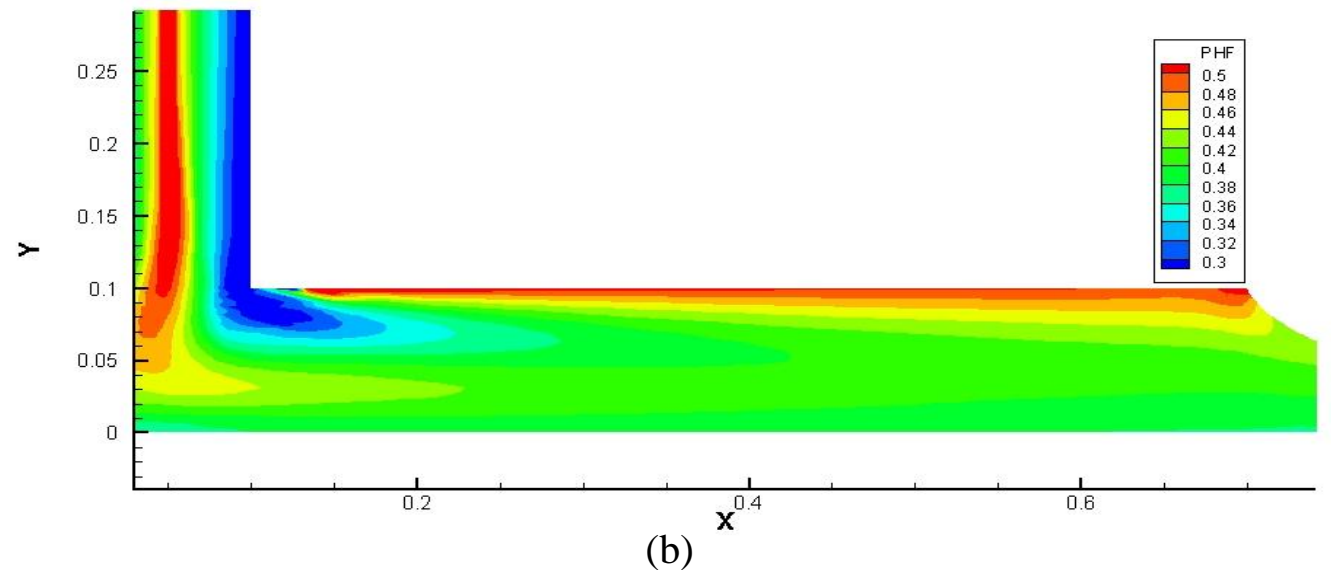

Figure 5.27: Two-dimensional particle distribution at coating bead for (a) $\mathrm{Ca}=0.1$ ("slots4-038") and (b) $\mathrm{Ca}=1.0$ ("slots4-060").

A plot of velocity profile at $x=0.40 \mathrm{~mm}$ is presented in Figure 5.28. The near vanishing shear rate at the die wall is clearly observed.

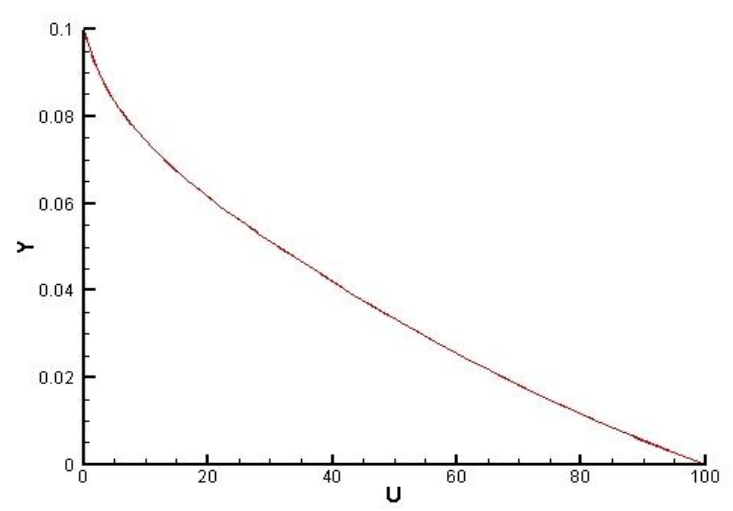

(a)

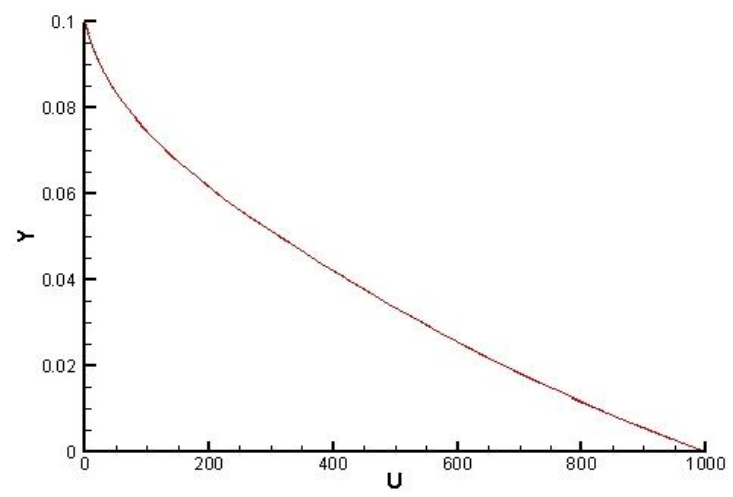

(b)

Figure 5.28: Velocity profile at $x=0.40 \mathrm{~mm}$ for (a) $\mathrm{Ca}=0.1$ ("slots4-038") and (b) $C a=1.0$ ("slots4-060"). 
The concentration profile at the same position $(x=0.40 \mathrm{~mm})$ is plotted in Figure 5.29. As indicated before, the concentration is independent of the substrate velocity. An important feature to observe is the high concentration reached at the slot die lip. For $t \approx H_{0} / 3$, the layer of high particle concentration is not located in the middle of the gap, but is attached to the wall. The concentration distribution along the wall die lip is presented in Figure 5.30. The distribution is virtually the same for both cases, except near the static contact line.

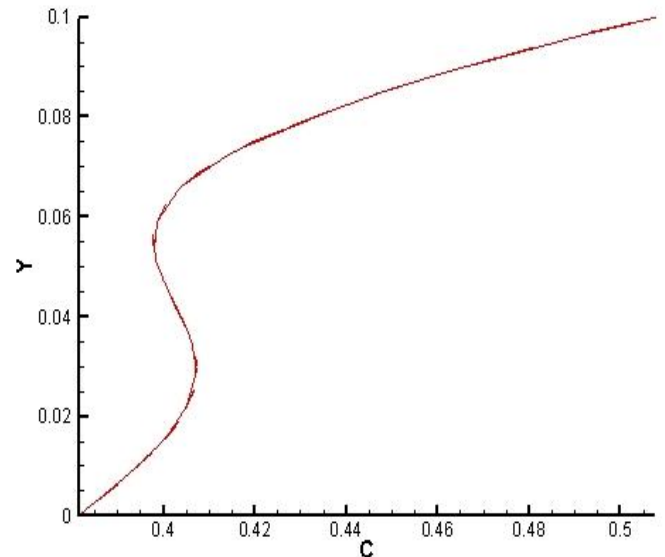

(a)

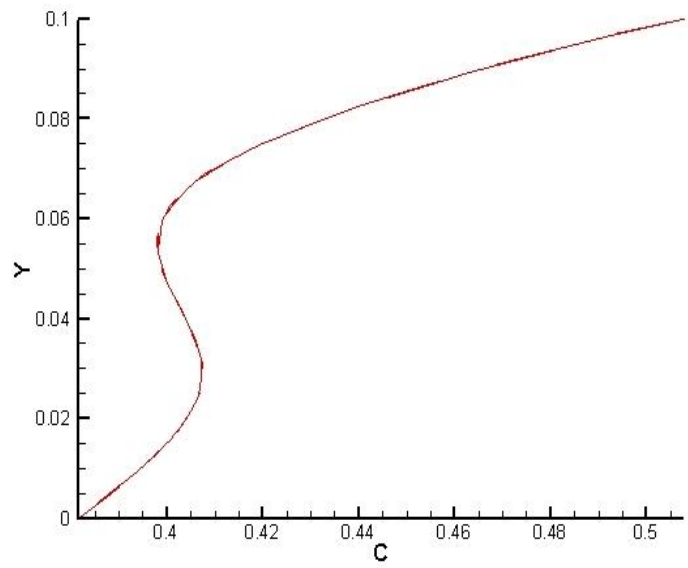

(b)

Figure 5.29: Concentration profiles at $x=0.40 \mathrm{~mm}$, for (a) $\mathrm{Ca}=0.1$ ("slots4-038") and (b) $C a=1.0$ ("slots4-060").

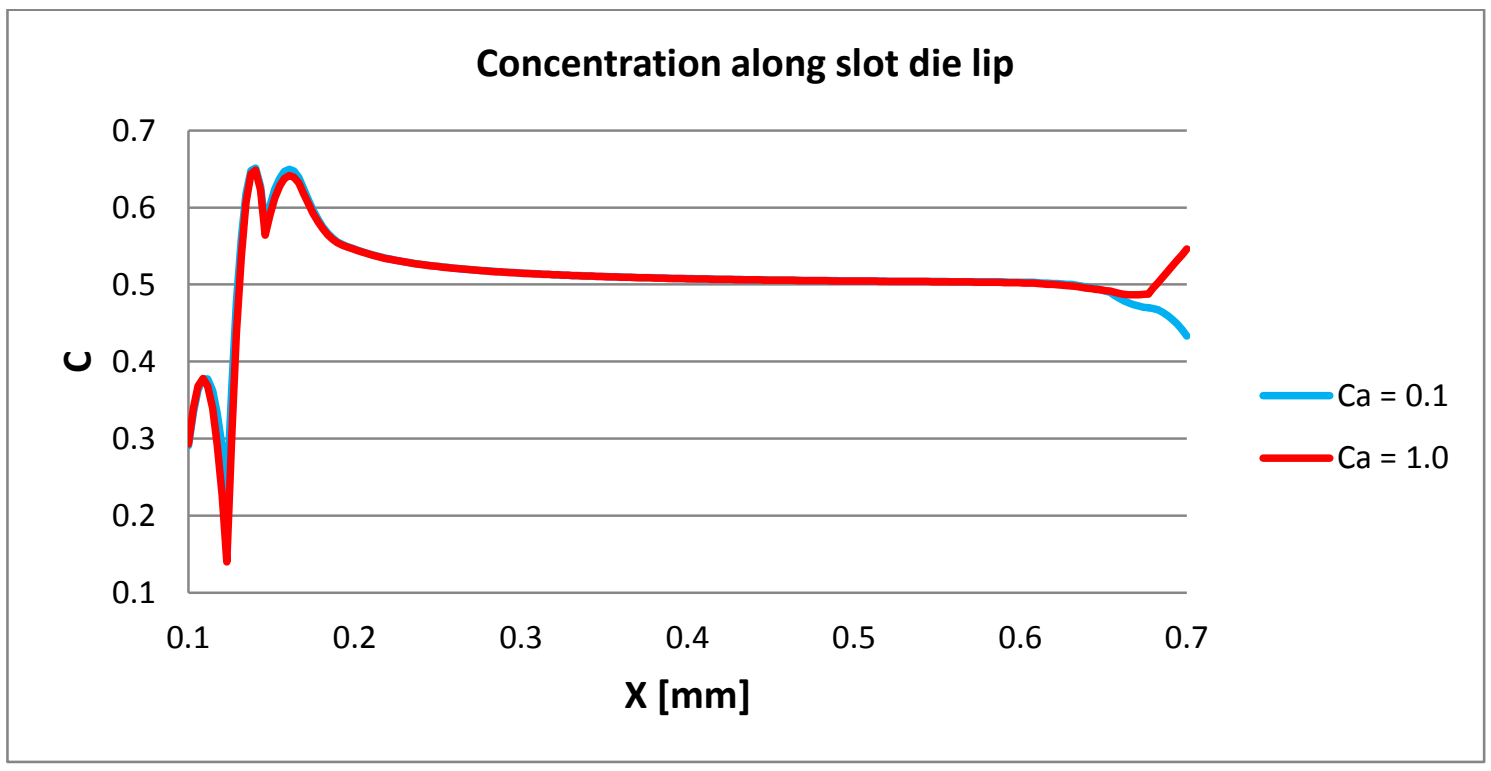

Figure 5.30: Concentration distribution along downstream slot die lip for $\mathrm{Ca}=0.1$ ("slots4038 ") and $C a=1.0$ ("slots4-060"). 
The pressure over the substrate for suspension and Newtonian flow for each capillary number is plotted in Figure 5.31 and Figure 5.32. The suspension flow presents a slightly higher pressure gradient, compared with their Newtonian counterparts, for both capillary numbers.

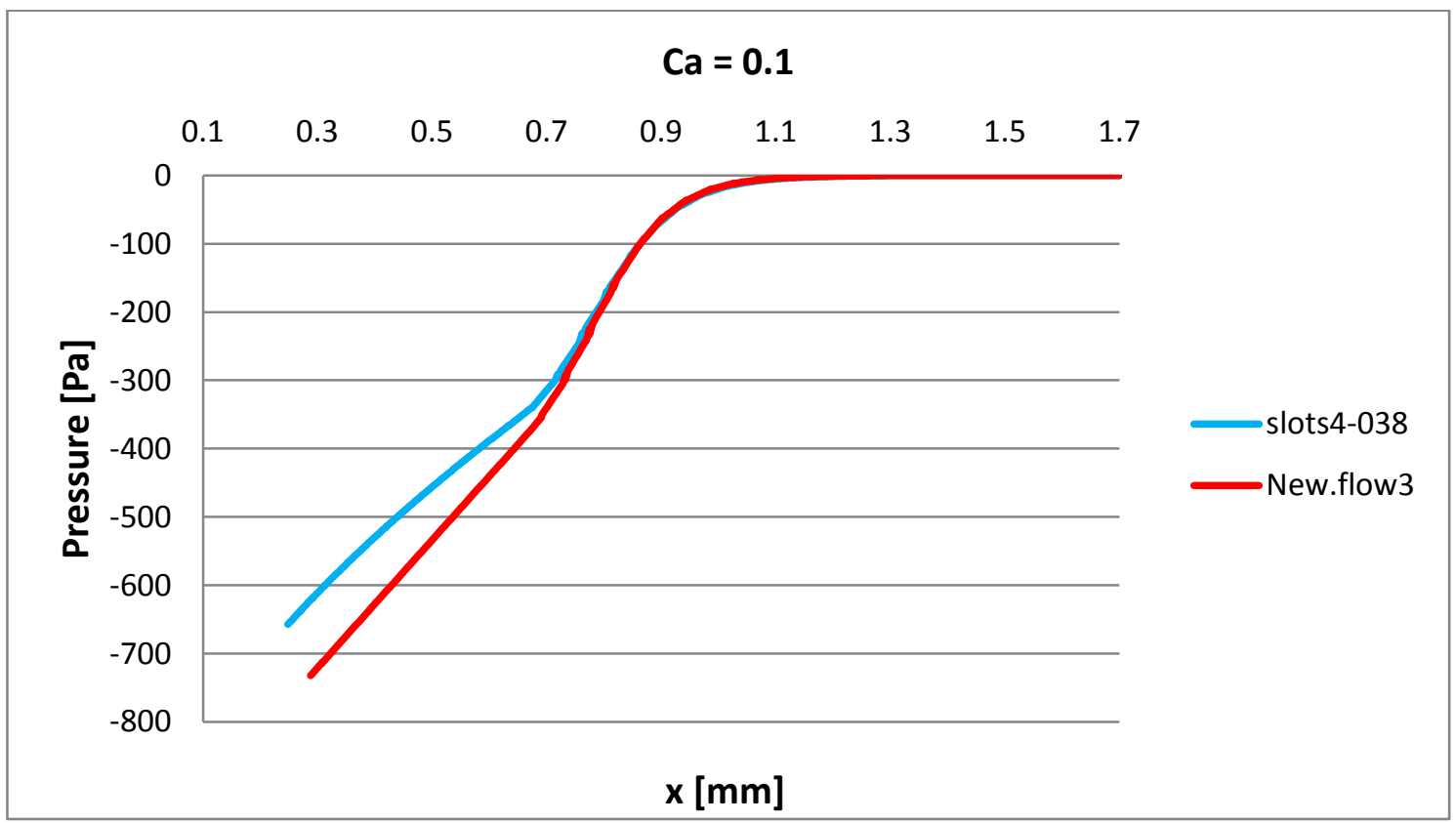

Figure 5.31: Pressure over substrate for $\mathrm{Ca}=0.10$.

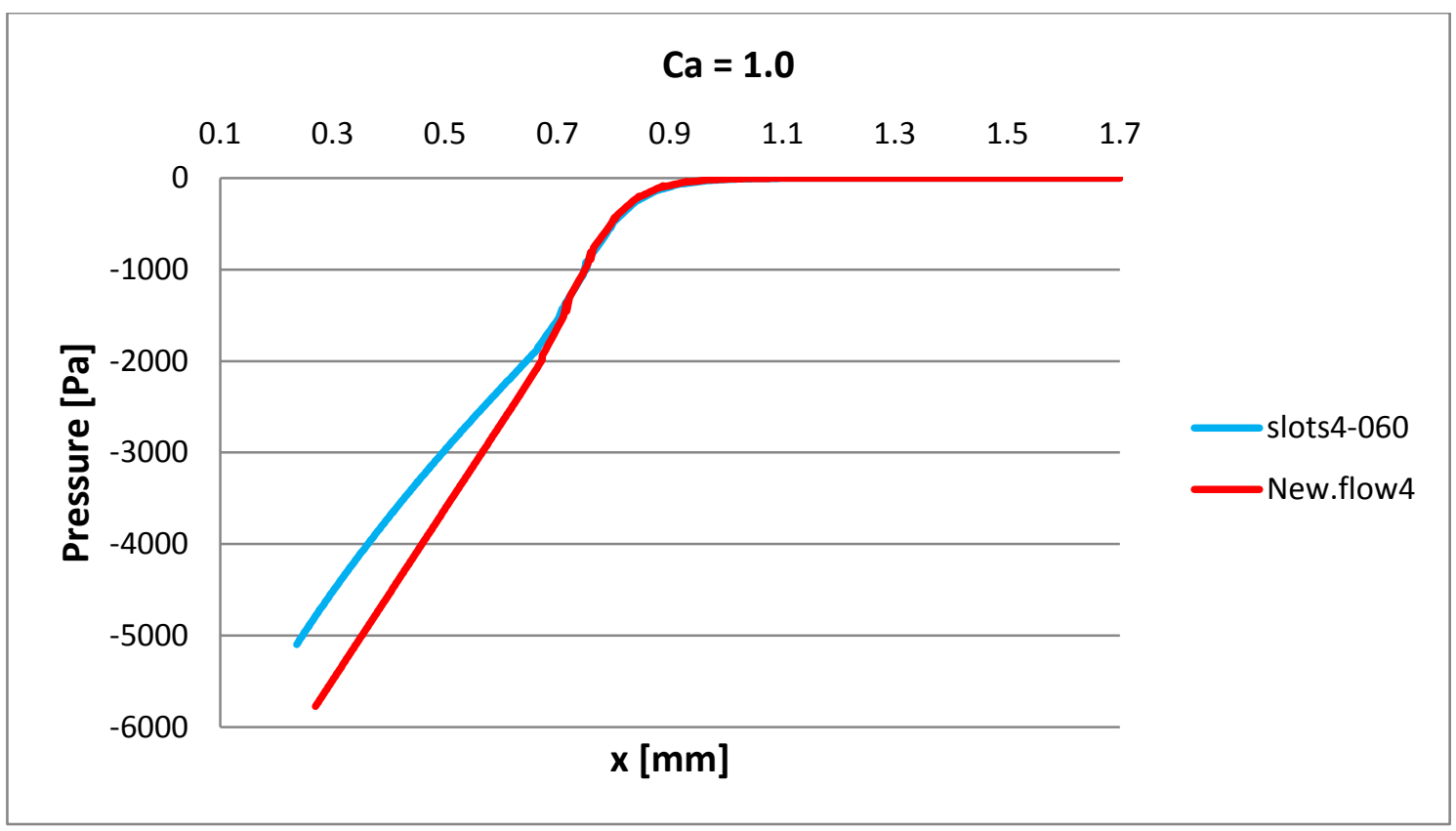

Figure 5.32: Pressure over substrate for $\mathrm{Ca}=1.0$ 


\section{Free surface}

A two-dimensional plot of concentration distribution in the film formation region is shown in Figure 5.33. The concentration scale was modified to have a better observation of the field. The high particle concentration near the die lip is convected to the top of the coated layer. Again, large gradients of particle concentration along the interface near the contact line are observed.

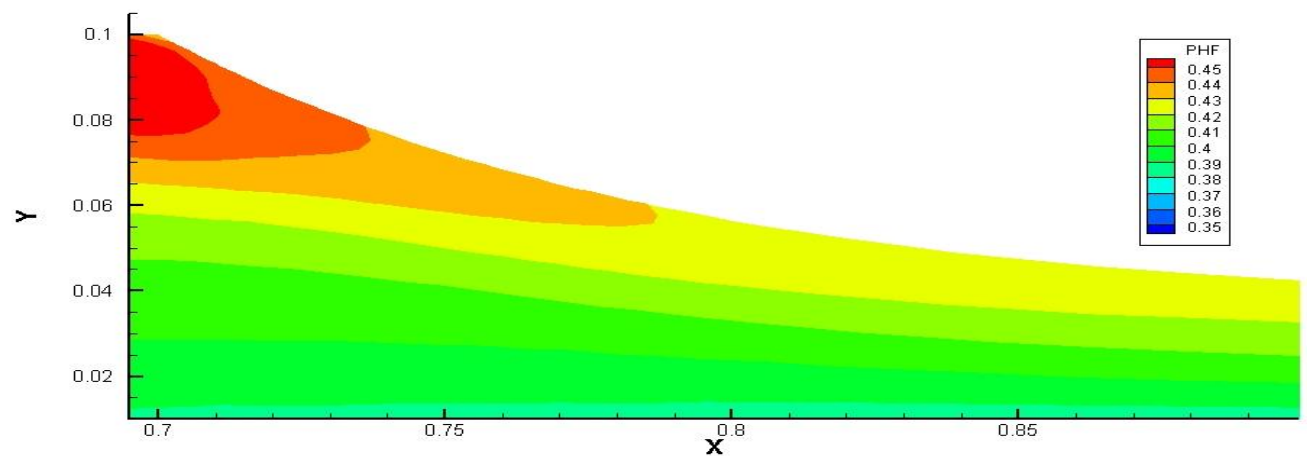

(a)

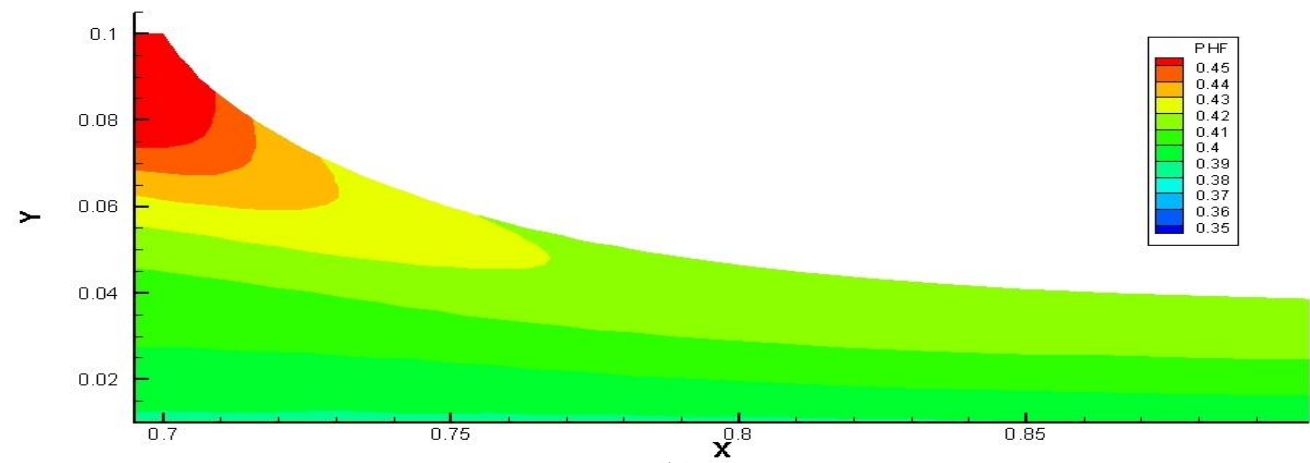

(b)

Figure 5.33: Concentration at free surface region for (a) $C a=0.1$ ("slots4-038") and (b) $C a=1.0$ ("slots4-060").

To get a real perspective of the particle suspension effects on the interfacial configuration, the interface shapes are compared for the two suspension flows, also with their respective Newtonian counterparts. After that, Figure 5.34 compares the interface profile for suspension and Newtonian flow at $\mathrm{Ca}=0.1$. The suspension interface is less curved than the Newtonian interface, in contrast with interface shape at $t=0.5 H_{0}$ (Figure 5.24). It is noted that the interface concentration and consequently the viscosity is higher than average values. 


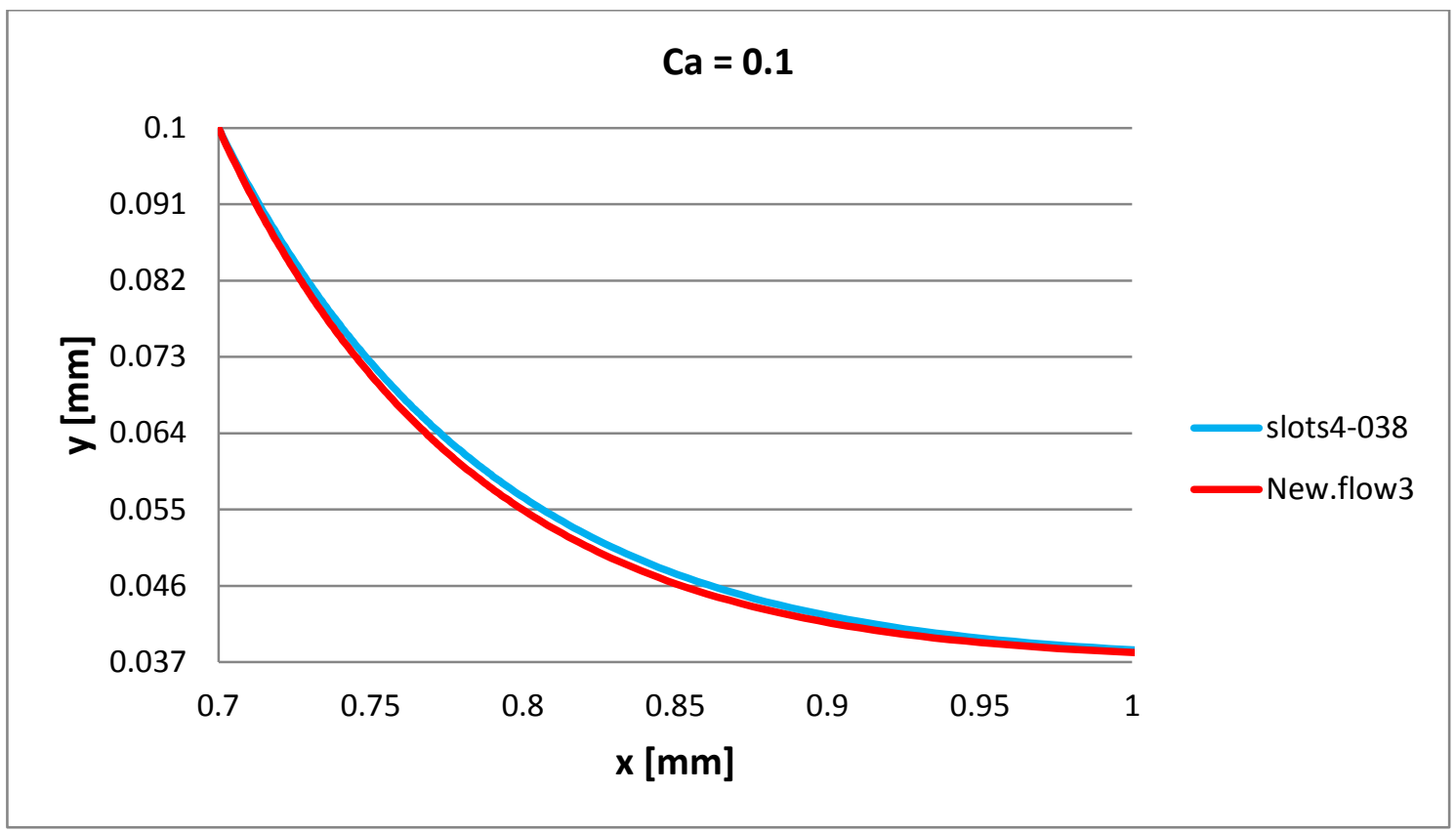

Figure 5.34: Comparison of free surface shape for $\mathrm{Ca}=0.1$ of suspension flow ("slots4038") and Newtonian flow (“New.flow3").

The effect on the interface configuration is stronger at high capillary numbers $C a=1.0$, as shown in Figure 5.35.

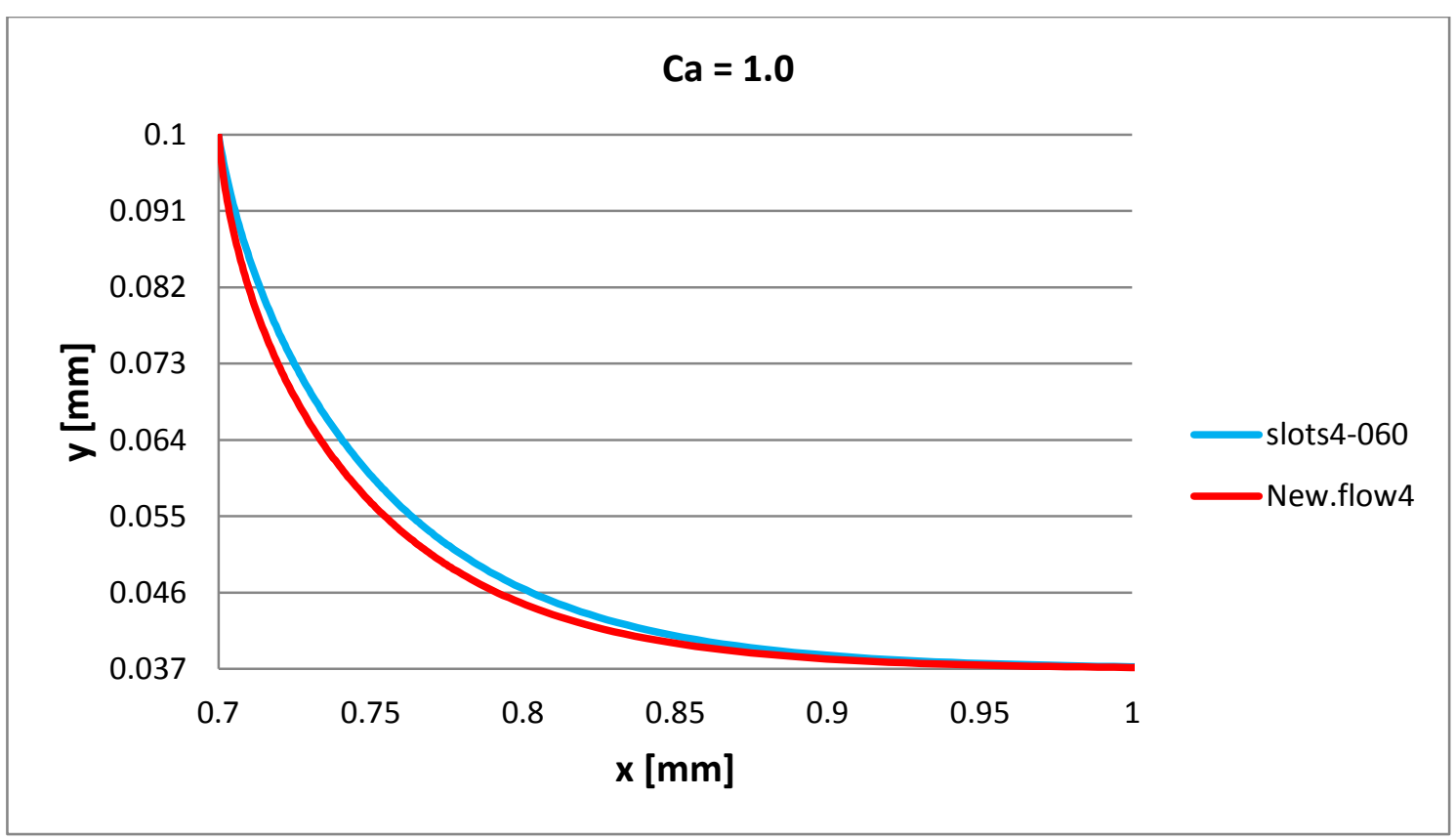

Figure 5.35: Comparison of free surface shape for $\mathrm{Ca}=1.0$ of suspension flow ("slots4060") and Newtonian flow ("New.flow4"). 
Particle concentration along interface length is plotted in Figure 5.36. Concentration remains higher than average concentration for both cases. In addition, higher concentration gradient near the contact line is observed for suspension flow at high capillary number, i.e. $\mathrm{Ca}=1.0$.

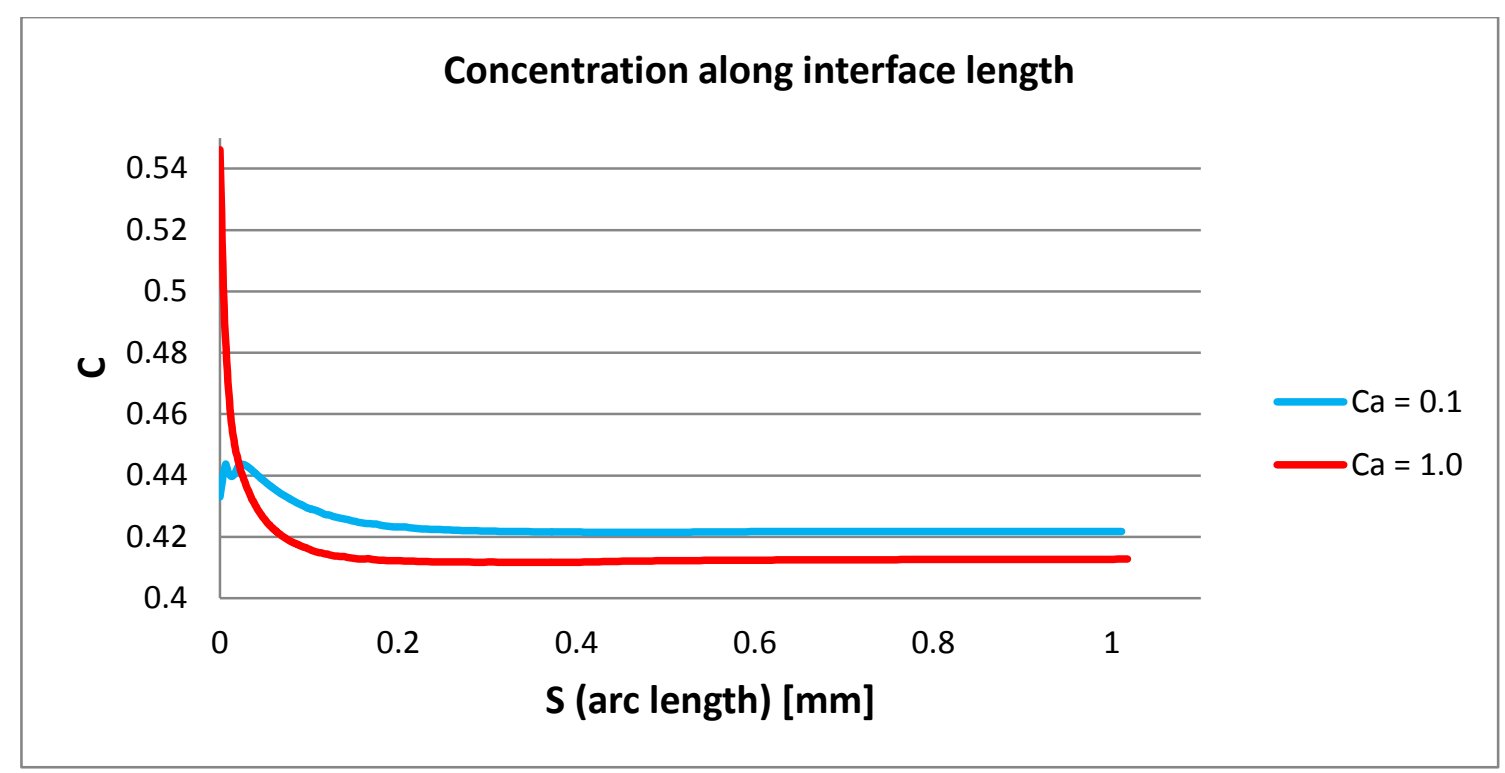

Figure 5.36: Concentration along the interface length for $\mathrm{Ca}=0.1$ ("slots4-038") and $\mathrm{Ca}=1.0$ ("slots4-060").

\section{Developed coated layer}

Figure 5.37 compares concentration profiles at a cross section of fully developed coated layer for the two cases. The results differ from the ones at $t=0.5 H_{0}$ (see Figure 5.26), that present a high concentration layer at the middle of the coated layer. Here, it is observed higher concentration at the top of the coated layer that results from particle convection from downstream slot die lip, as observed in Figure 5.38. 


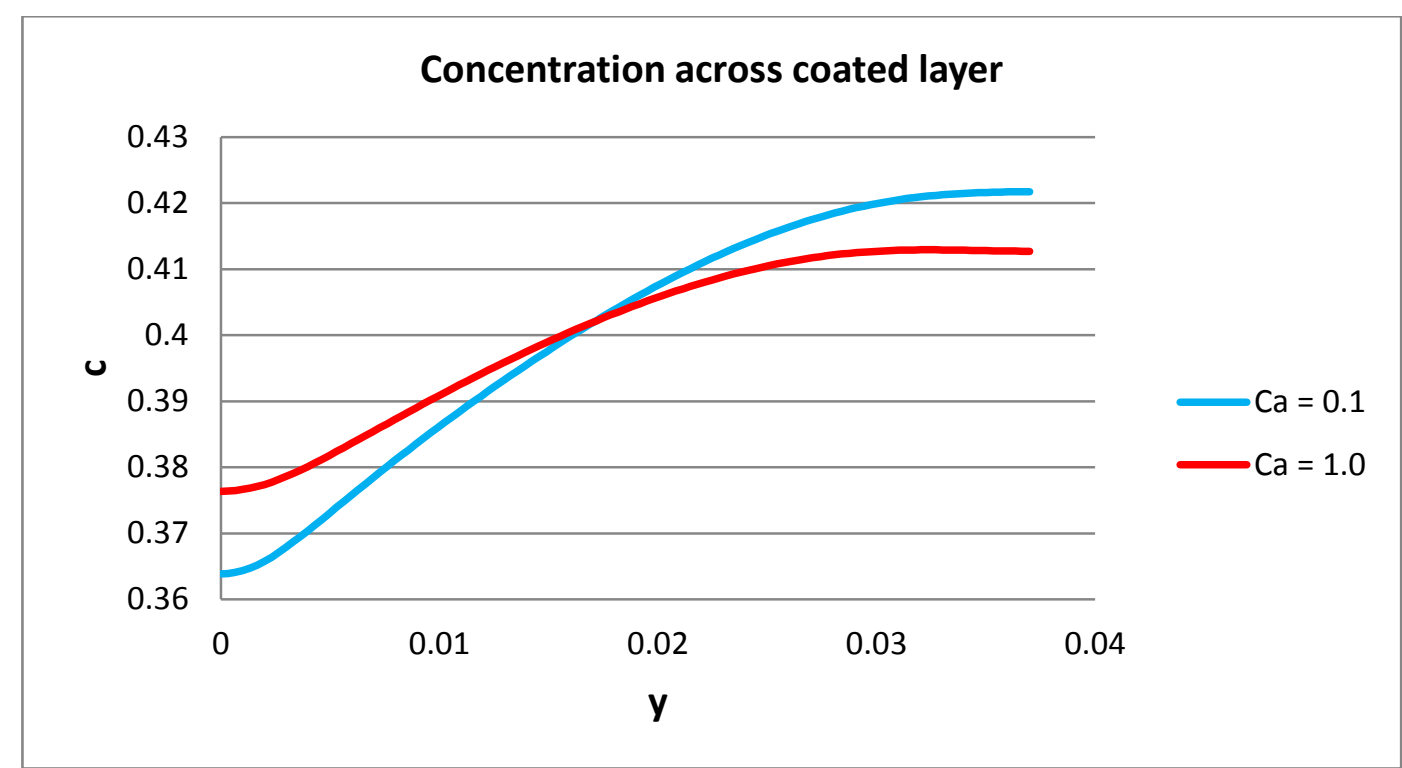

Figure 5.37: Particle concentration across coated layer for $\mathrm{Ca}=0.1$ ("slots4-038") and $\mathrm{Ca}=1.0$ ("slots4-060").

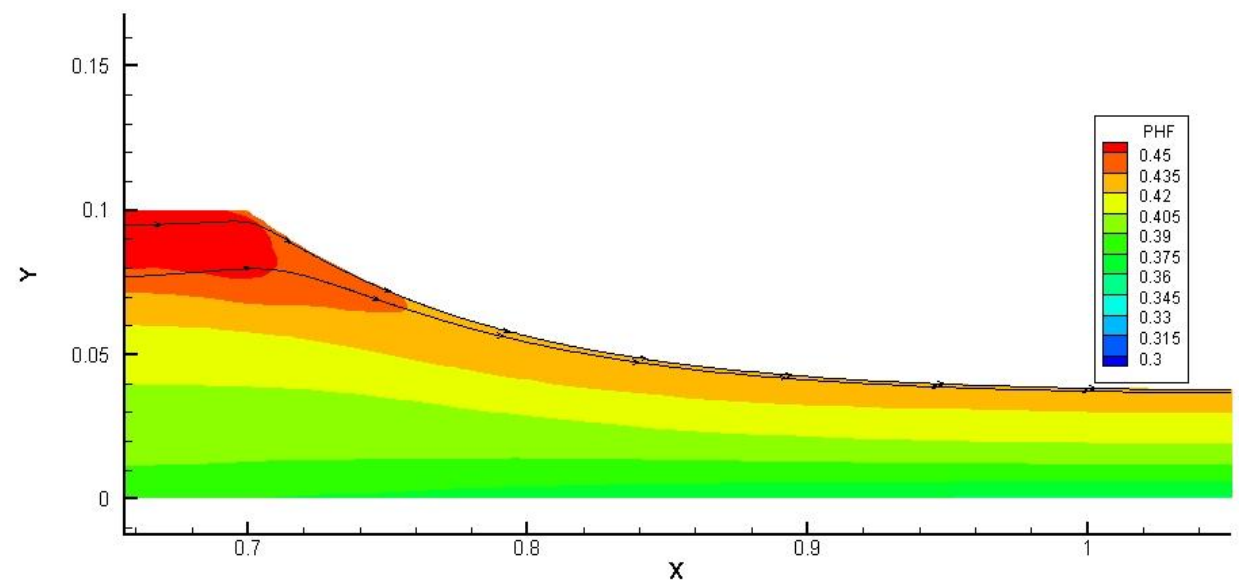

Figure 5.38: Particles convected to coated layer top.

It is important to note that concentration distribution across coated layer was changed through diminishing the film thickness.

\subsection{3.}

\section{Analysis when Recirculation Flow is Present}

Finally, it is presented results for simulation of slot coating when recirculation flow is present. This flow pattern appears when flow rate (then film thickness) is low enough so that a backflow is needed to maintain the flow rate at 
downstream coating bead. In other words, the Poiseuille contribution is high, and then a higher pressure gradient than in previous cases is expected.

As it was observed high concentration values are expected in regions with low shear-rate, then it is important to identify where in the domain this occurs. Again, because of Poiseuille contribution to the backflow, there is a streamline where shear-rate vanishes.

Simulations were performed for different film thickness close to Newtonian case low-flow limit. Convergence problems appear due to an increment in particle concentration above maximum packing concentration at downstream static contact line. Despite convergence problems, it was possible to obtain a simulation were high particle migration is observed, and concentration at feed slot exit is close to reach developed profile. It is presented the result for suspension flow with parameters shown in Table 5.6.

Table 5.6: Parameters for slot coating simulation when recirculation flow is present.

\begin{tabular}{|c|c|}
\hline Case & slots4-092 \\
\hline Bulk viscosity & 0.060 Pa.s \\
\hline Substrate velocity & $100.0 \mathrm{~mm} / \mathrm{s}$ \\
\hline Surface tension & $0.060 \mathrm{~kg} / \mathrm{s} 2$ \\
\hline Capillary number & $\mathbf{0 . 1 0}$ \\
\hline Continuous phase viscosity & $0.0119 \mathrm{~Pa} . \mathrm{s}$ \\
\hline Particle concentration & 0.40 \\
\hline Particle radius & $4.0 \mu \mathrm{m}$ \\
\hline Gap size of slot $\left(H_{s}\right)$ & $0.10 \mathrm{~mm}$ \\
\hline Gap size of coating bead $\left(H_{0}\right)$ & $0.10 \mathrm{~mm}$ \\
\hline Film thickness $(\mathrm{t})$ & $0.014 \mathrm{~mm}$ \\
\hline$k_{c}$ & 0.5 \\
\hline$k_{\eta}$ & 0.75 \\
\hline
\end{tabular}

\section{Coating bead}

Suspension flow at coating bead is presented in Figure 5.39. It could be observed the recirculation region, with high concentration in it, and coated layer streamlines. The accumulation of particles is clearly observed in the recirculation region. Besides, low particle concentration is observed at downstream die lip. 


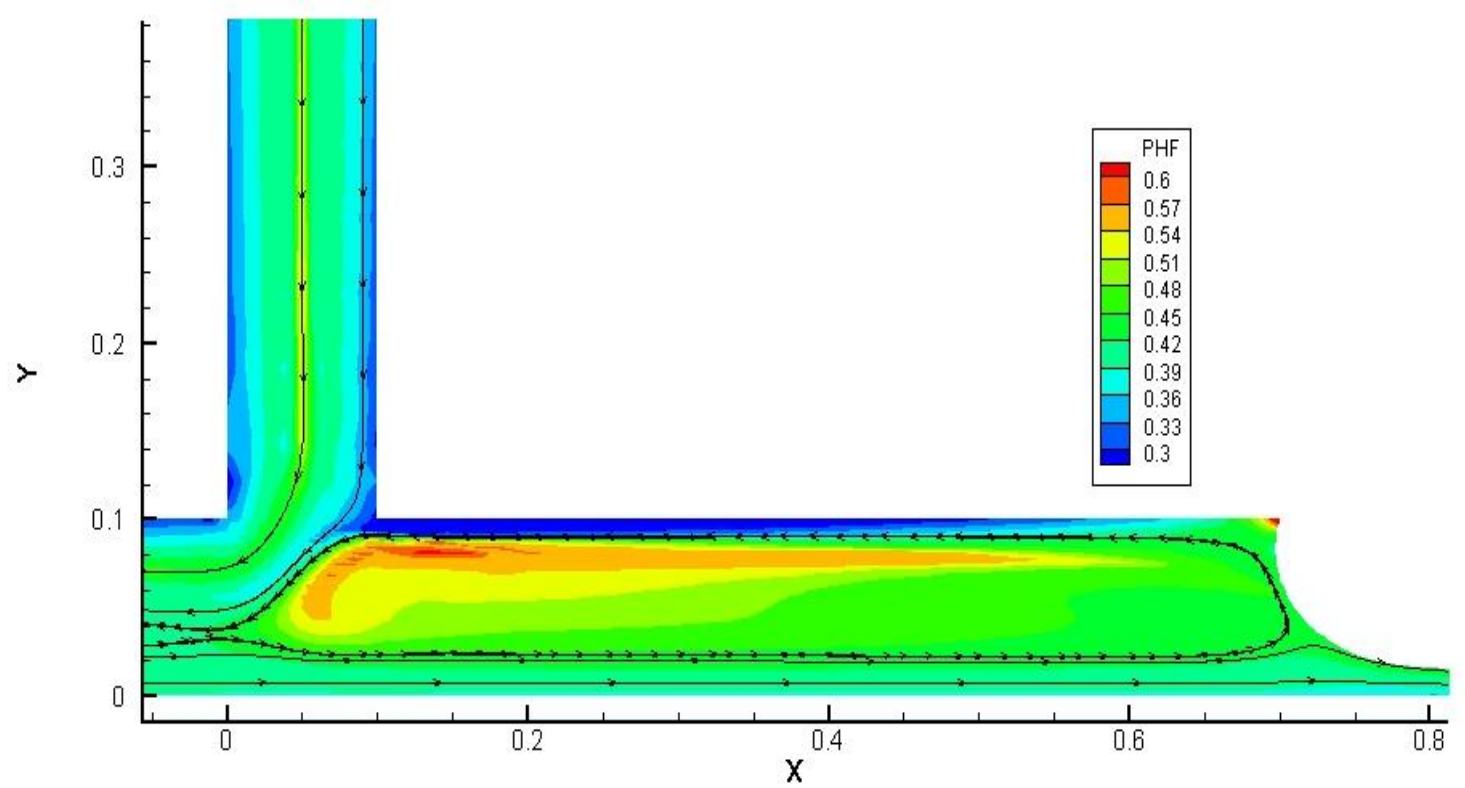

Figure 5.39: Two-dimensional particle distribution at coating bead for $\mathrm{Ca}=0.1$ ("slots492").

A closer view of recirculation region around $x=0.15 \mathrm{~mm}$ is plotted in Figure 5.40. The velocity field is plotted, and then maximum concentration is observed where maximum velocity is localized. The flow in this region is like a flow between parallel plates with maximum velocity near $y=0.08 \mathrm{~mm}$.

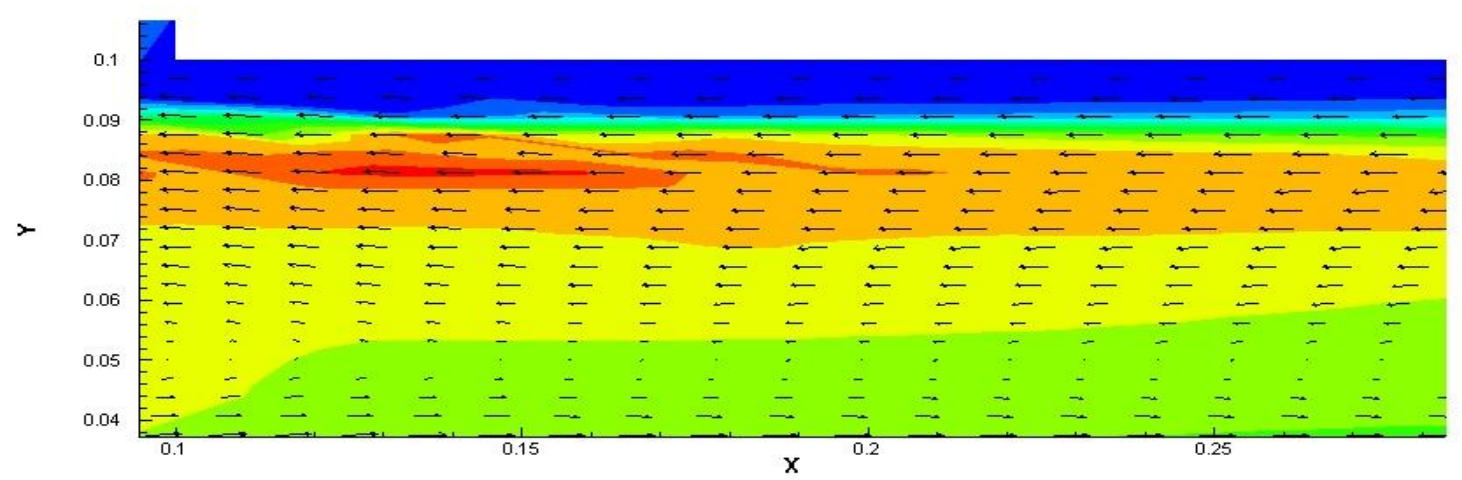

Figure 5.40: Closer view of recirculation region.

Because of the recirculation region the suspension that is deposited over the substrate has to flow upstream, as it is observed for the four streamlines of Figure 5.41. In addition, the centerline streamline of the feed slot diminishes its concentration along the flow, and then it is not expected high particle concentration at the center of the coated layer. 


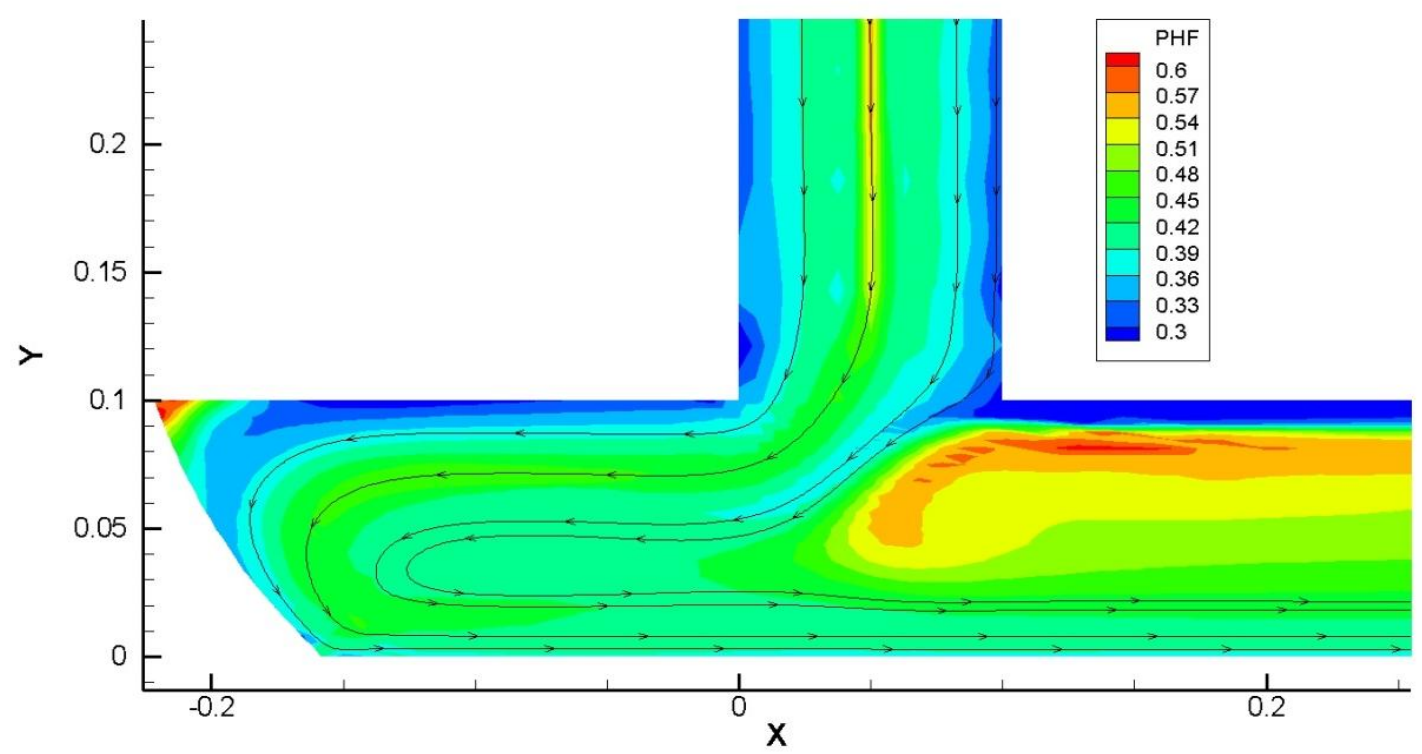

Figure 5.41: Streamlines for coated layer.

The velocity and concentration profiles across the coating bead at coordinate $x=0.3 \mathrm{~mm}$ are presented in Figure 5.42. As expected, higher backflow velocity is around $y=0.08 \mathrm{~mm}$, and then zero velocity gradient (Figure 5.42-a). Figure 5.42-b depicts concentration profile, with high particle concentration around $y=0.08 \mathrm{~mm}$. Another interesting feature is the concentration value at slot die lip, lower than concentration at the wall for fully developed Poiseuille flow between parallel plates. Flow in this zone has Poiseuille and Couette flow contributions, then particle concentration profile, and concentration at the walls are different.

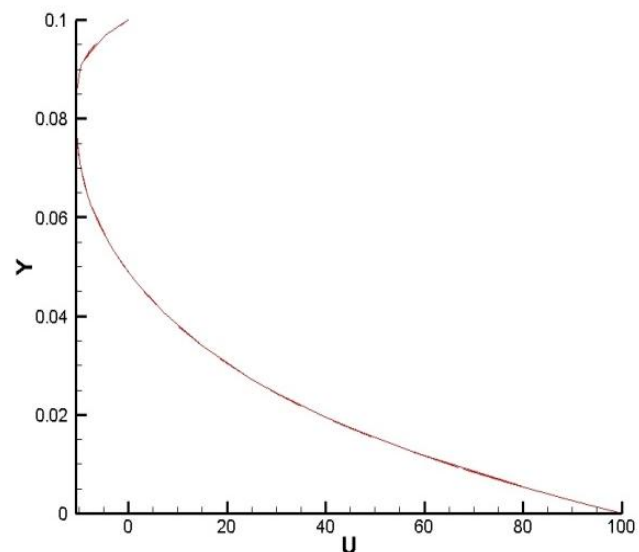

(a)

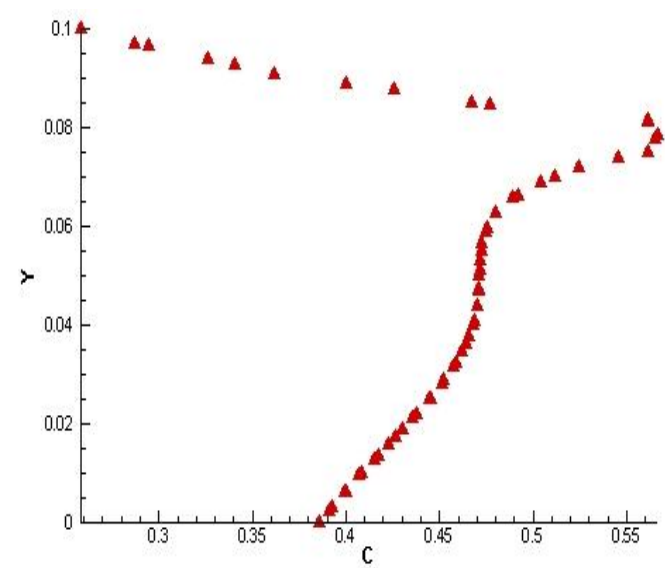

(b)

Figure 5.42: (a) Velocity and (b) concentration profiles at $x=0.3 \mathrm{~mm}$. 
Figure 5.43 compares the pressure over the substrate for suspension and Newtonian flow at same capillary number $(\mathrm{Ca}=0.1)$. There is virtually the same pressure distribution over the substrate.

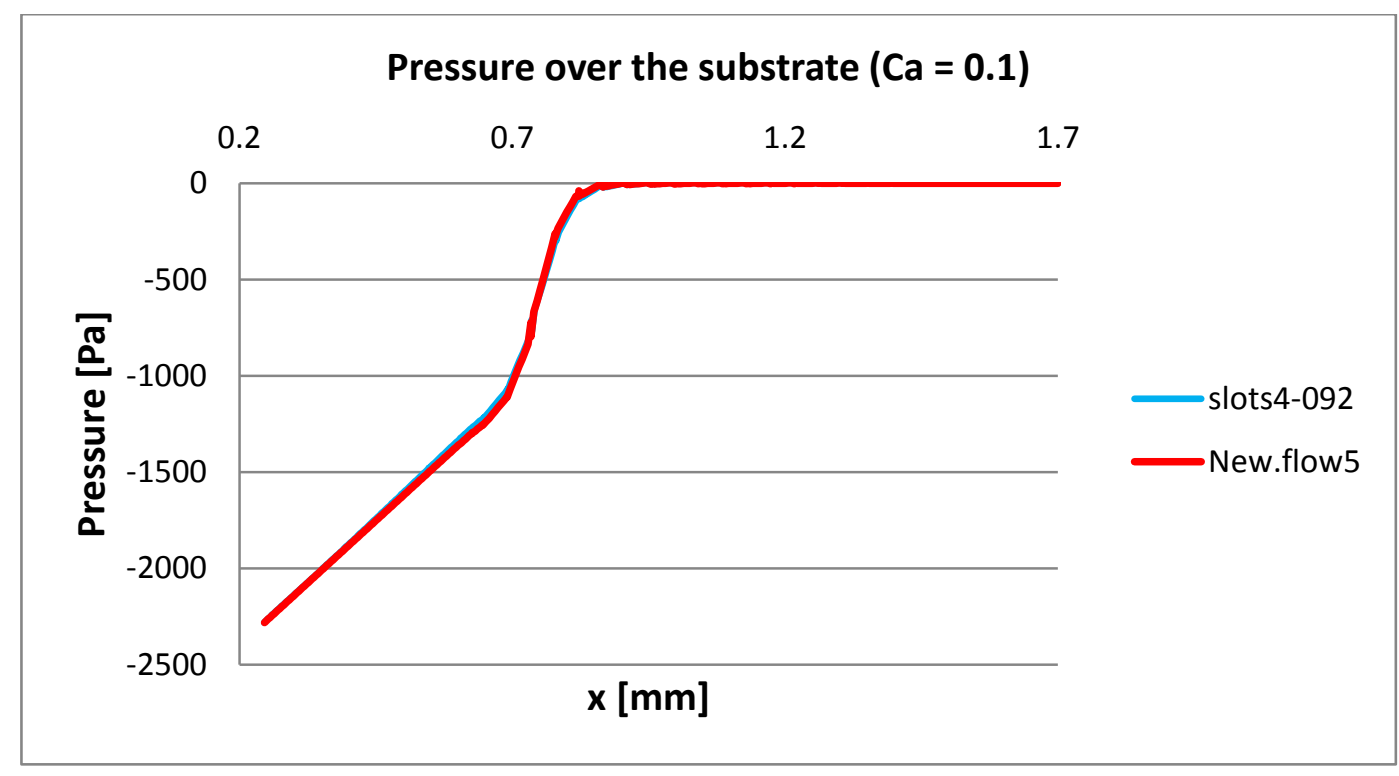

Figure 5.43: Pressure over the substrate for case "slots4-092".

\section{Free surface}

Figure 5.44 depicts the two-dimensional plot of concentration distribution in the film formation region. The recirculation region has higher concentration values than the suspension above, at coating bead, and then suspension with high concentration is convected to the top layer of the coated film. The results show that particle concentration along the interface is higher than the average concentration; besides large concentration gradients close to the contact line can be observed. 


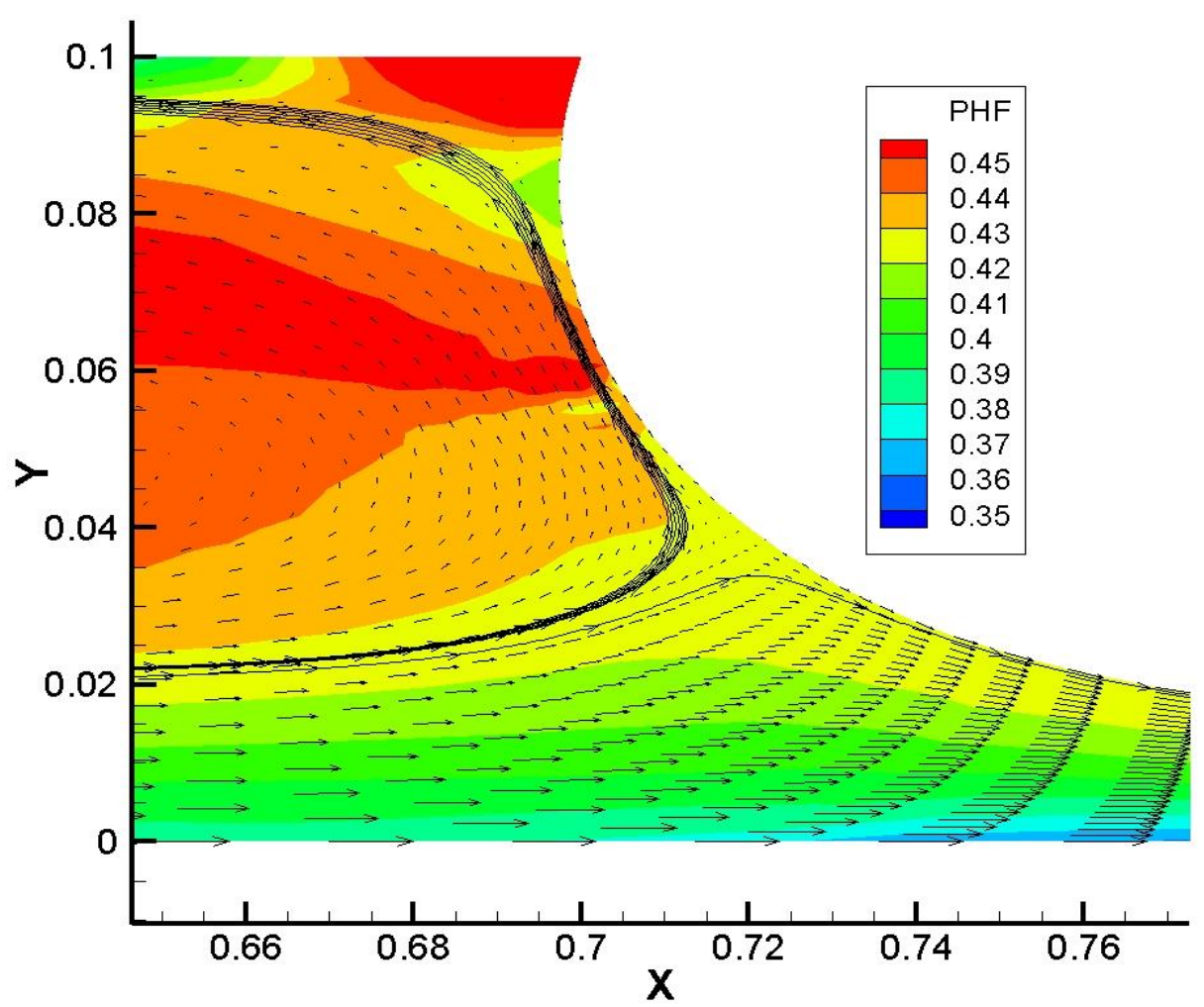

Figure 5.44: Concentration at free surface region for case "slots4-092".

In Figure 5.45 the free surface shape is compared with the corresponding shape of a Newtonian flow for the same capillary number. As observed in previous simulation results (section 5.4.2), it is also observed that the interface for a suspension is less curved than its Newtonian interface counterpart. It is important to mention that this behavior is observed when the concentration at interface is higher than average concentration $(c=0.4)$. A possible explanation for this phenomenon comes from the stress balance at the interface: basically, the interface curvature compensates the difference between pressure and viscous stresses (see equation 2.22). If the viscous term is augmented, this difference is reduced and smaller curvature is enough to close the balance. 


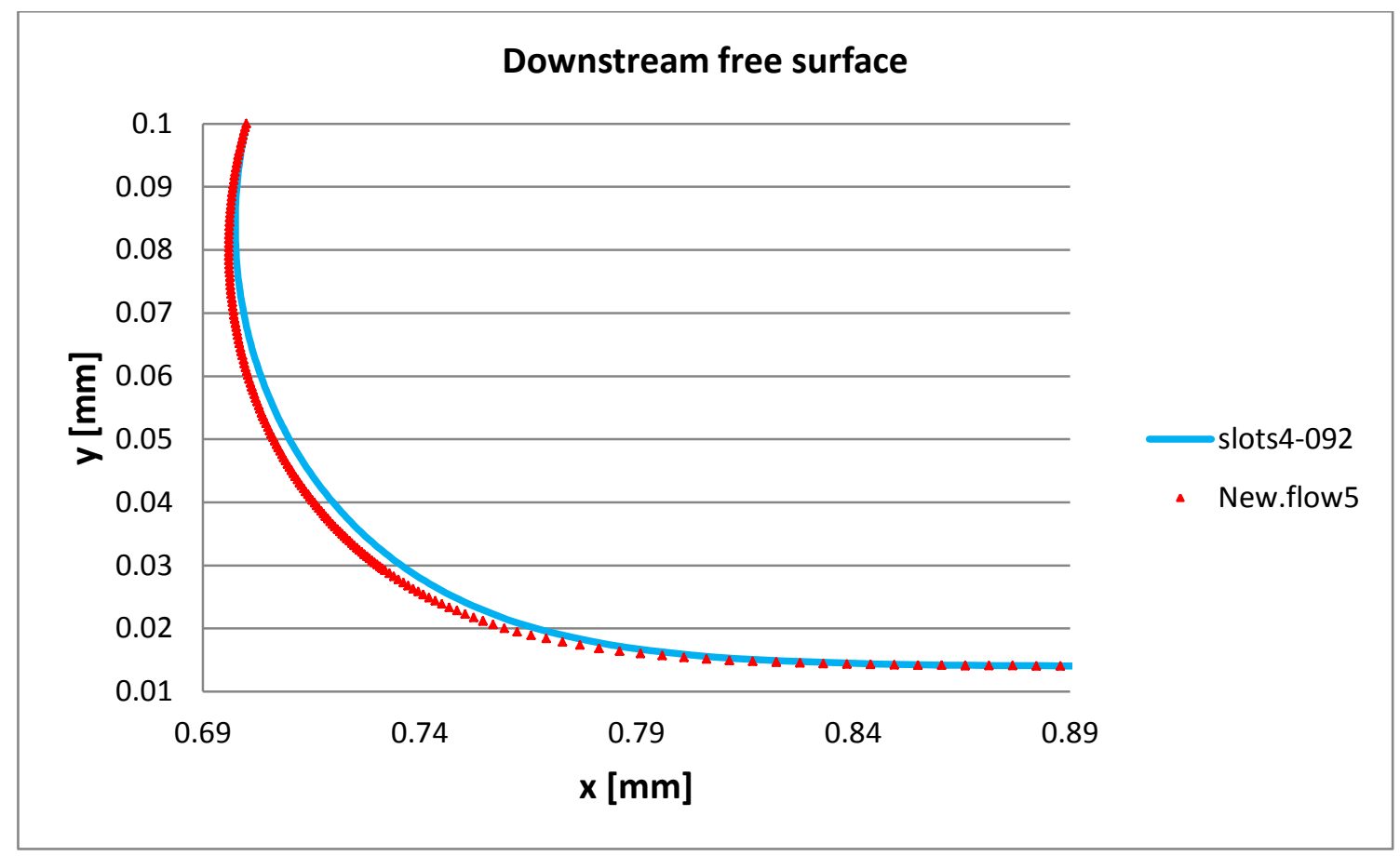

Figure 5.45: Free surface shape for Newtonian liquid and suspension ("slots4-092").

The concentration of particles along the interface length is plotted in Figure 5.46. Again, it is observed a high gradient concentration near the static contact line, where the particle concentration is close to the maximum packing fraction. Then, the concentration reaches its developed value slightly above the bulk concentration. Hence, it is expected higher concentration at top layers of the final coated layer.

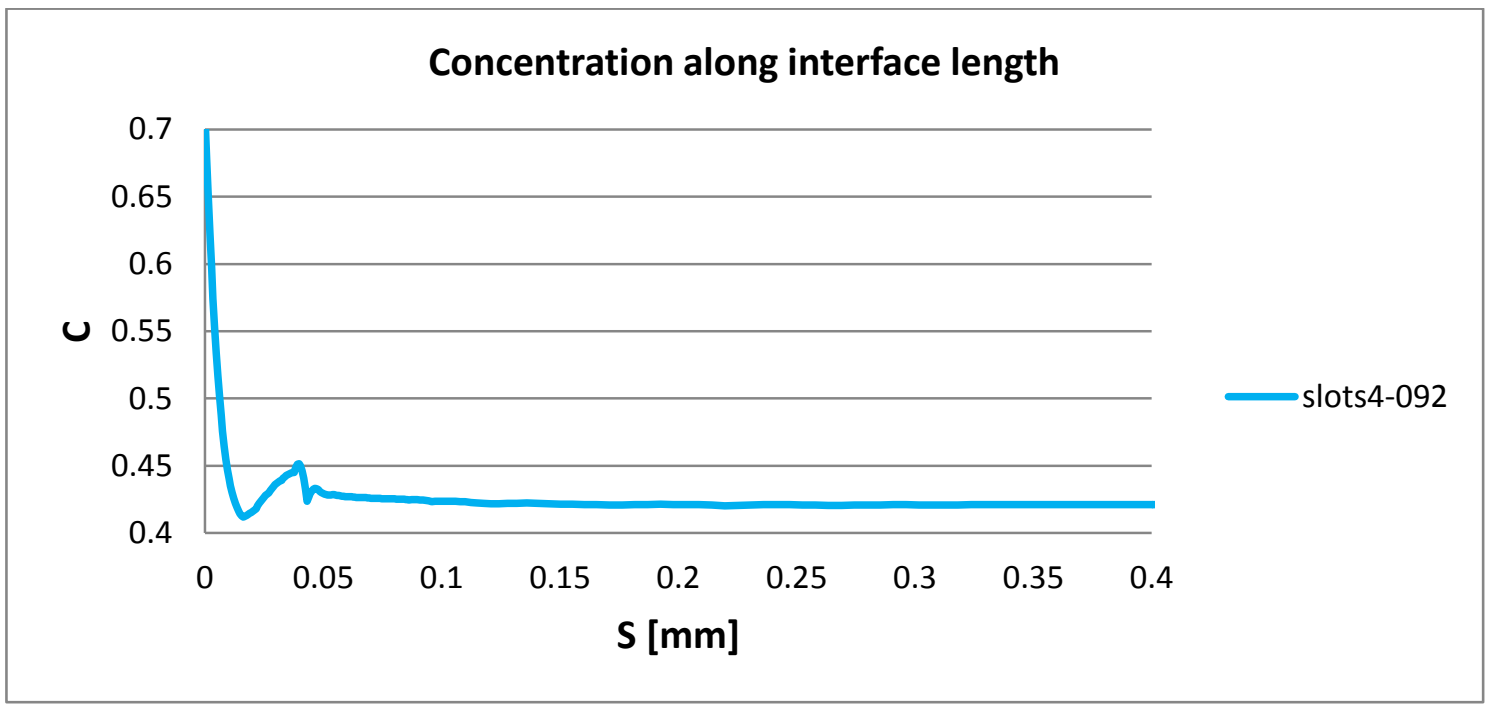

Figure 5.46: Concentration along interface length for case "slots4-092". 
In addition, the suspension flow modifies slightly the position of the stagnation point compared with a Newtonian flow. Figure 5.47 depicts the location of this point for both flows.

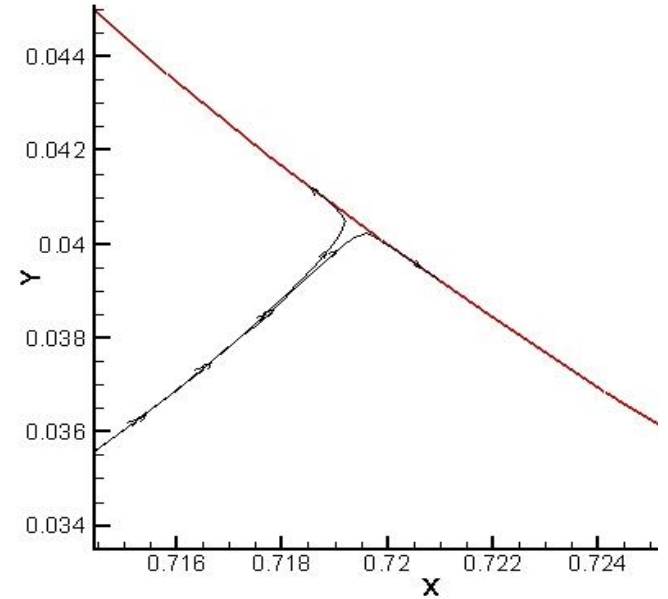

(a)

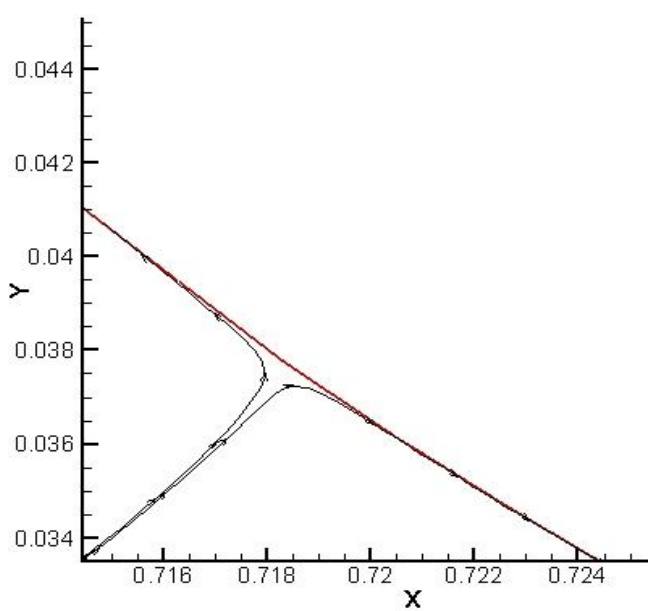

(b)

Figure 5.47: Location of stagnation point for (a) suspension flow ("slots4-092") and (b) Newtonian flow.

Another plot that helps visualize the stagnation point is the tangential velocity along the interface, as plotted in Figure 5.48. This point is localized where the suspension velocity changes sign. Additionally, it is observed that the developed flow is reached close to $s=0.3 \mathrm{~mm}$.

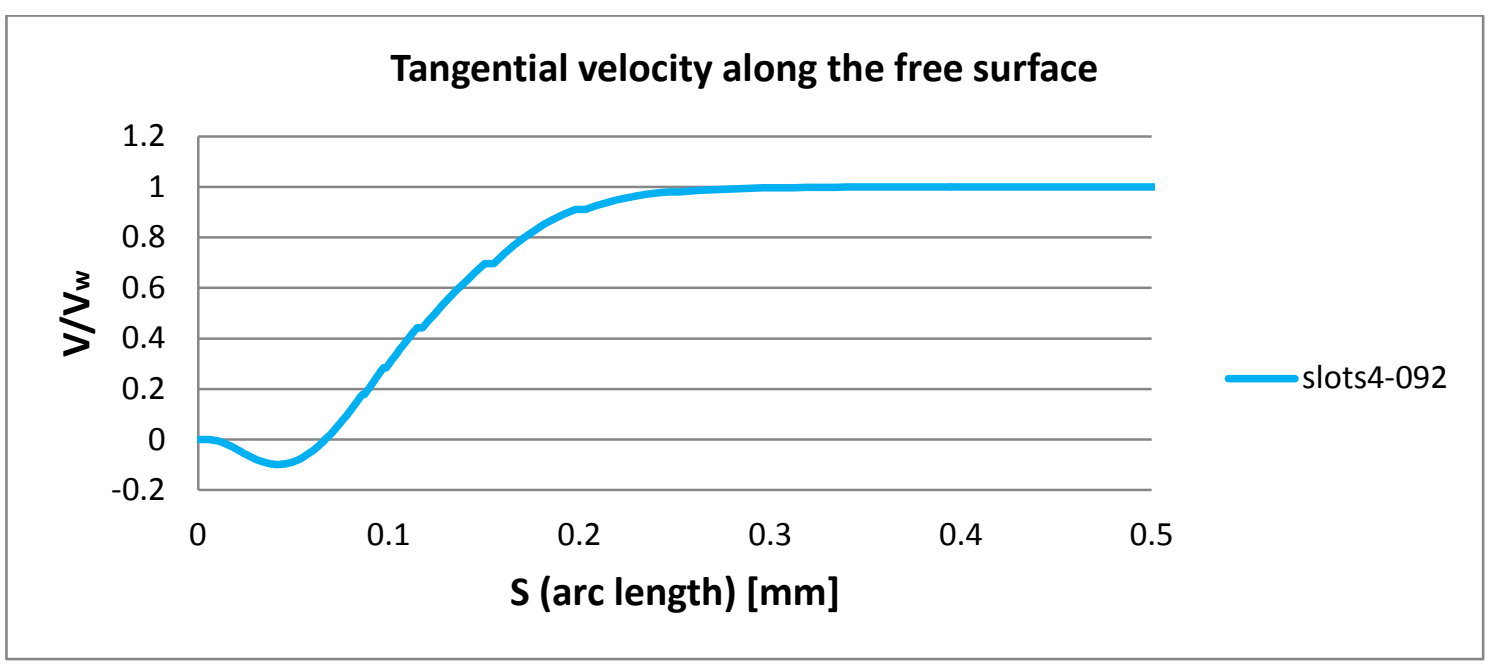

Figure 5.48: Tangential velocity along the free surface for case "slots4-092". 


\section{Developed coated layer}

Figure 5.49 depicts particle concentration across developed coated layer. It is observed higher concentration values at the top of the film. As plotted in Figure 5.44 , the streamlines with high concentration are the ones close to recirculation region, where the concentration is above average value. This concentration profile is similar to the one presented when recirculation flow starts (section 5.4.2). Then no significant change in concentration profile is expected for intermediate values of film thickness.

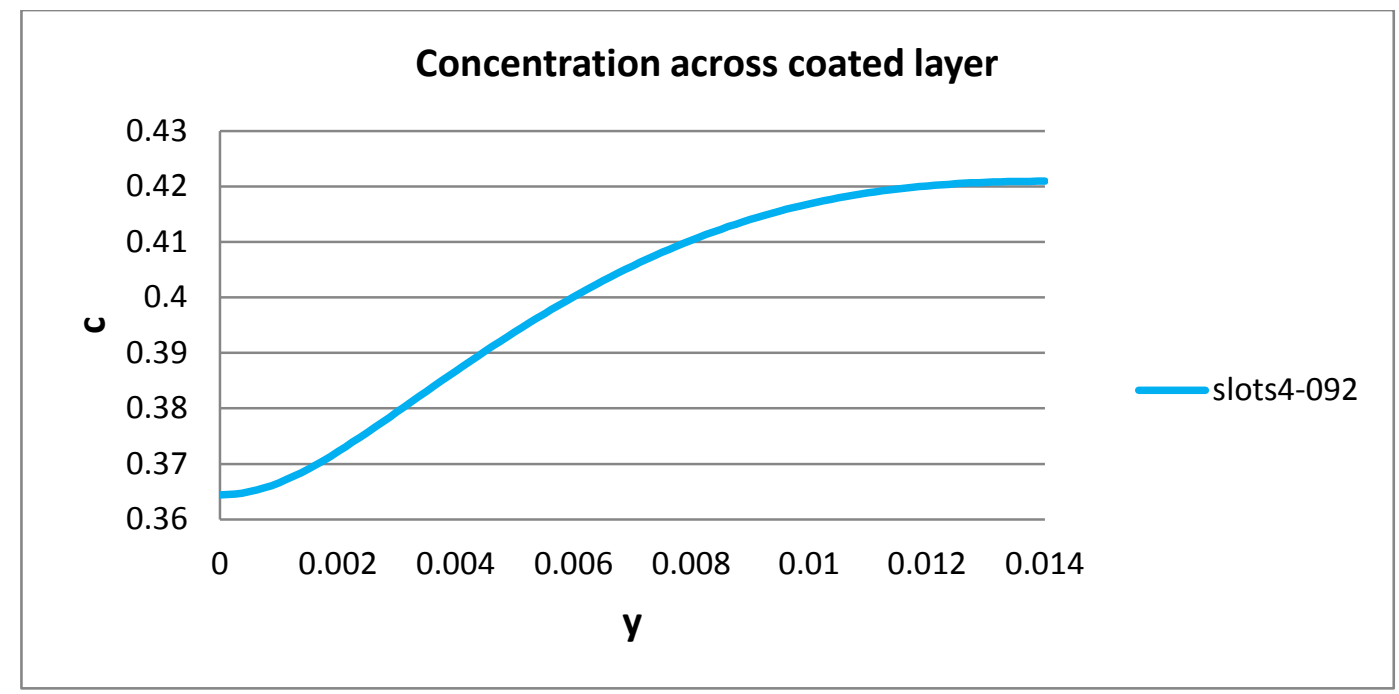

Figure 5.49: Particle concentration across coated layer for case "slots4-092".

\section{5.}

Influence of Film Thickness on Coated Layer Concentration Profile

Simulations for different film thickness were performed using the same values for the parameters of Table 5.4. The flow rate, consequently the film thickness, was diminished gradually. The fully developed particle concentration profile at the coated film as a function of the film thickness is plotted in Figure 5.50 . 


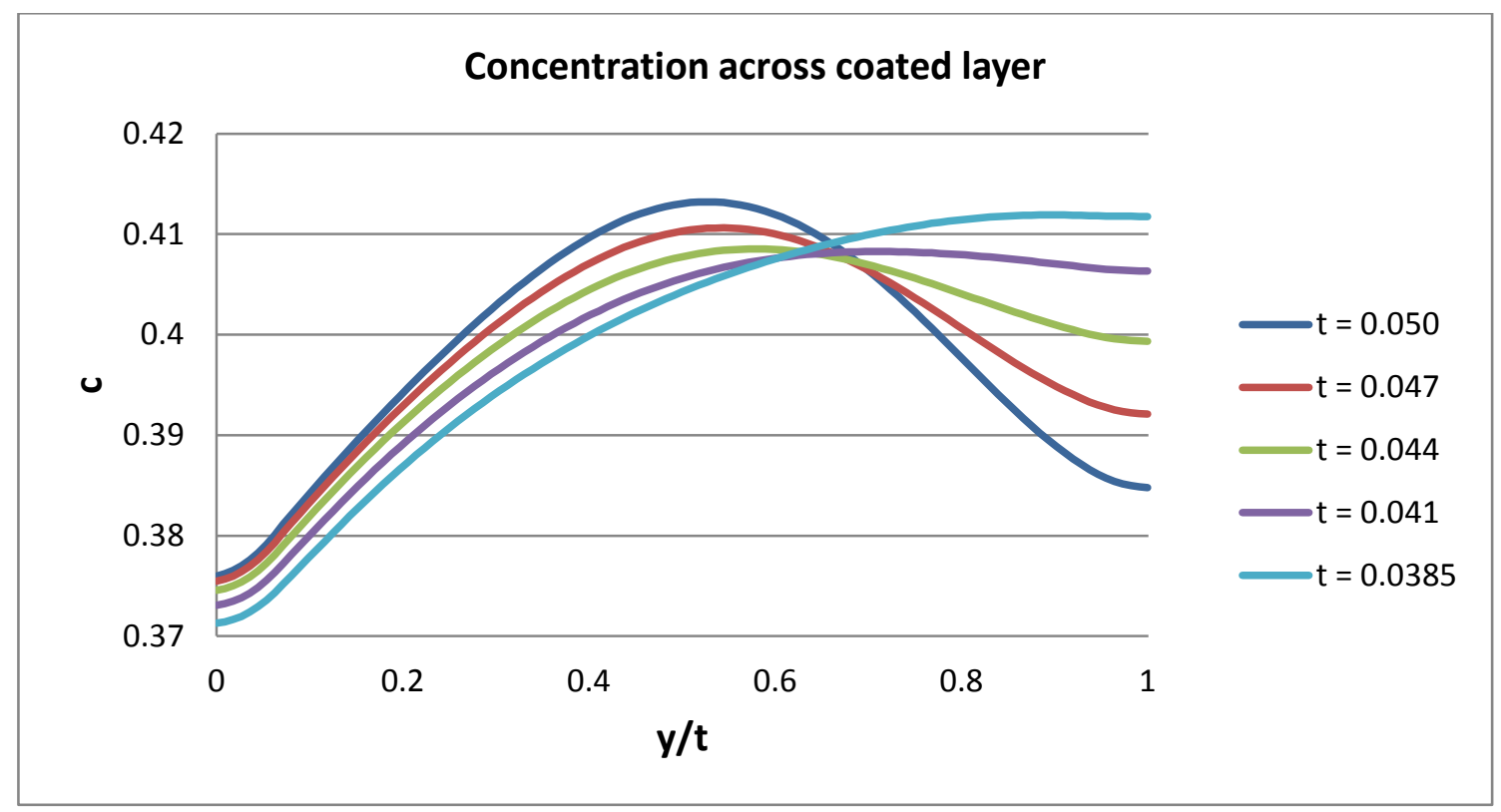

Figure 5.50: Fully developed concentration profile at different film thickness.

As explained in section 5.4, when diminishing the film thickness starting from $t=H_{0} / 2$, the shear-rate at downstream slot die lip diminishes, consequently particles migrate towards that zone. Therefore, high concentration streamlines near slot die lip flow to top layer of the coated layer. Figure 5.50 gives a clear idea of the concentration profile for different film thickness. The particle concentration at the substrate is virtually the same, while the concentration at the top layer varies. All this simulation cases were performed without recirculation under the downstream die lip. 


\section{6 \\ Conclusions}

\section{1. Conclusions}

Slot coating process considering a particle suspension as the coating liquid was studied. The continuity, momentum and particle transport equations describe the flow and particle concentration fields. This free surface problem was solved using the Galerkin/Finite Element Method. The generated set of algebraic nonlinear equations was solved using the Newton's method.

Simulation in full domain slot coating flow was performed, showing the concentration fields. It was observed a good qualitative agreement at identifying regions of high concentration, which are located where shear rate vanishes. Maximum packing particle concentration was always observed at both static contact lines. Besides, at upstream maximum packing concentration could be reach.

The slot coating flow simulations capture particle migration when the velocity profile changes from nearly parabolic, at the end of feed slot, to a plug flow at developed coated layer. It was observed that migration depends on the ratio of film thickness to coating gap $\left(t / H_{0}\right)$. No uniform distribution of particles at coated layer was found. When film thickness is half of coating gap, a layer of high particle concentration is localized at the center of the deposited coated layer. Lower film thickness, such that the velocity gradient at downstream slot die lip was close to zero, led to a high particle concentration at the wall, which was convected to the top layer of the final coated film. This leads to different particle concentration configurations at the final coated layer.

When the film thickness is diminished to a point where recirculation flow appears, it was found that higher concentration values are localized in recirculating flow. The particle concentration profile at the coated layer was found to be similar to case when shear rate is close to zero at downstream slot die lip. 
The results clearly show that an analysis of the coating process of particle suspension should include particle transport. The hypothesis that the particle concentration is constant within the flow is not valid. Non-uniform particle concentration profile in the coated film may have a strong impact on the drying process.

\section{2.}

\section{Future Works}

The following recommendations are proposed, as future works, in order to improve the present numerical model and to expand the scope of this study.

The length of downstream slot die can be varied in order to see the influence on particle diffusion at different thickness to gap ratio $t / H_{0}$, and on concentration distribution at downstream interface and coated layer.

Improvement of the prediction of the present model can be studied in order to avoid increment of particle concentration above maximum packing fraction in zones where suspension velocity and shear rate are close to zero. The static contact lines are example of this. Apparently, this was the main problem to get converged solutions in this research. With this problem solved, low-flow limits of suspension in slot coating flow could be explored.

Further study on migration coefficients can be made, in order to determine the adequate geometry to obtain a desired concentration profile at the coated layer.

An expansion of the model can be made by considering the surface tension as a function of particle concentration. The results showed concentration gradient at downstream interface, so that Marangoni effects cannot be negligible and could have a deep impact on the flow field, the interface shape and, consequently on the low-flow limit of the process.

Other particle migration models can also be explored, in order to analyze which one will give the best description of the process.

Finally, experimental studies should be performed to measure particle concentration distribution, failure mechanisms, interfaces shape and low-flow limit. This will provide a better understanding of particle migration in very narrow channels. 


\section{References}

ABBOTT, J. R. et al. Experimental observations of particle migration in concentrated suspensions: Couette flow. Journal of Rheology, Vol. 35, No. 5, pp. 773-795, 1991.

ALTOBELLI, S. A.; GIVLER, R. C.; FUKUSHIMA, E. Velocity and concentration measurements of suspensions by nuclear magnetic resonance imaging. Journal of Rheology, Vol. 35, No. 5, pp. 721-734, 1991.

BARNER, H. A. A handbook of elementary rheology. The University of Wales Institute of Non-Newtonian Fluid Mechanics, University of Wales Aberystwyth, Penglais, Aberystwyth, Dyfed, Wales, 2000.

BEGUIN, A. E. Method of coating strip material. U.S. Patent 2681294, 1954.

BENJAMIN, D. F. Roll Coating Flows and Multiple Rolls Systems. PhD thesis, University of Minnesota, Minneapolis, MN, USA, 1994.

BIRD, R. B.; ARMSTRONG, R. C.; HASSAGER, O. Dynamics of polymeric liquids: Fluid mechanics, volume 1. John Wiley \& Sons, New York, USA, 1977.

CARVALHO, M. S. Deformable Roll Coating in Rigid and Deformable Gaps. PhD thesis, University of Minnesota, Minneapolis, MN, USA, 1996.

CARVALHO, M. S.; KHESHGI, H.S. Low-Flow Limit in Slot Coating: Theory and Experiments. AIChE Journal, Vol. 46, No. 10, pp. 19071917, 2000. 
CHRISTODOULOU, K. N.; SCRIVEN, L. E. Discretization of free surface flows and other moving boundary problems. Journal of Computational Physics, Vol. 99, No. 1, pp. 39-55, 1992.

EINSTEIN, A. Eine neue bestimmung der Moleküldimensionen. Annalen der Physik, Vol. 324, No. 2, pp. 289-306, 1906.

FANG, Z.; PHAN-THIEN, N. Numerical simulation of particle migration in concentration suspensions by a finite volume method. Journal of Non-Newtonian Fluid Mechanics, Vol. 58, No. 1, pp. 67-81, 1995.

GALADA-MARIA, F.; ACRIVOS, A. Shear-Induced Structure in a Concentrated Suspension of Solid Spheres. Journal of Rheology, Vol. 24, No. 6, pp. 799-814, 1980.

GATES, I. A. Slot Coating Flows: Feasibility, Quality. PhD thesis, University of Minnesota, Minneapolis, MN, USA, 1999.

GRAHAM, A. L.; MAMMOLI, A. A.; BUSCH, M. B. Effects of demixing on suspension rheometry. Rheologica Acta, Vol. 37, No. 2, pp. 139-150, 1998.

HOFFMAN, R. L. Discontinuous and Dilatant Viscosity Behavior in Concentrated Suspensions. I. Observation of a Flow Instability. Transactions of the Society of Rheology, Vol. 16, No. 1, pp. 155-173, 1972.

KISTLER, S. F.; SCHWEIZER, P. M. Liquid film coating. Chapman \& Hall, 1997.

HIGGINS, B. G.; SCRIVEN, L. E. A new mixed finite element method for computing viscoelastic flows. Chemical Engineering Science, Vol. 35, No. 3, pp. 673-682, 1980.

HOOD, P. Frontal solution program for unsymmetric matrices. International Journal for Numerical Methods in Engineering, Vol. 10, No. 2, pp. 379-399, 1976. 
KARNIS, A.; GOLDSMITH, H. L.; MASON, S. G. The Kinetics of Flowing Dispersions I. Concentrated Suspensions of Rigid Particles. Journal of Colloid and Interface Science, Vol. 22, No. 6, pp. 531-553, 1966.

KIM, J. M.; LEE, S. G.; KIM, C. Numerical simulations of particle migration in suspension flows: Frame-invariant formulation of curvature-induced migration. Journal of Non-Newtonian Fluid Mechanics, Vol. 150, No. 2-3, pp. 162-176, 2008.

KRIEGER, I. M. Rheology of monodispersed latices. Advances in Colloid and Interface Science, Vol. 3, No. 2, pp. 111-136, 1972.

KRISHNAN, G. P.; BEIMFOHR, S.; LEIGHTON, D. T. Shear-induced radial segregation in bidisperse suspensions. Vol. 321, pp. 371-393, 1996.

LEE, A. G.; SHAQFEH, E. S. G.; KHOMAMI, B. A study of viscoelastic free surface flows by the finite element method: Hele-Shaw and slot coating flows. Journal of Non-Newtonian Fluid Mechanics, Vol. 108, No. 1-3, pp. 327-362, 2002.

LEIGHTON, D. Migration arising from gradients in shear stress particle distributions in Poiseuille flow. NASA Conference Publication 3006, pp. 109-124, 1988.

LEIGHTON, D.; ACRIVOS, A. Measurement of shear-induced selfdiffusion in concentrated suspensions of spheres. Journal of Fluid Mechanics, Vol. 177, pp. 109-131, 1987a.

LEIGHTON, D.; ACRIVOS, A. The shear-induced migration of particles in concentrated suspensions. Journal of Fluid Mechanics, Vol. 181, pp. 415-439, 1987b.

LYON, M. K.; LEAL, L. G. An experimental study of the motion of concentrated suspensions in two-dimensional channel flow. Part 1. Monodisperse systems. Journal of Fluid Mechanics, Vol. 363, pp. 25-56, 1998. 
MCTIGUE, D. F.; JENKINS, J. T. Channel flow of a concentrated suspension. In Advances in Micromechanics of Granular Materials, pp. 381-481, 1992.

MIN, K. H.; KIM, C. Simulation of particle migration in free-surface flows. AIChE Journal, Vol. 56, No. 10, pp. 2539-2550, 2010.

MILLER, R. M.; MORRIS, J. F. Normal stress-driven migration and axial development in pressure-driven flow of concentrated suspensions. Journal of Non-Newtonian Fluid Mechanics, Vol. 135, No. 2-3, pp. 149-165, 2006.

MILLS, P.; SNABRE, P. Rheology and structure of concentrated suspensions of hard spheres, shear induced particle migration. Journal de Physique II, Vol. 5, No. 10, pp. 1597-1608, 1995.

NOTT, P. R.; BRADY, J. F. Pressure-driven flow of suspensions: simulation and theory. Journal of Fluid Mechanics, Vol. 275, pp. 157199, 1994.

PANTON, R. L. Incompressible Flow. $3^{\text {rd }}$. ed., Hoboken, New Jersey: John Wiley \& Sons, 2005. 821p.

PASQUALI, M.; SCRIVEN, L. E. Free surface flows of polymer solutions with models based on the conformation tensor. Journal of Non-Newtonian Fluid Mechanics, Vol. 108, No. 1-3, pp. 363-409, 2002.

PHILLIPS, R. J. et al. A constitutive equation for concentrated suspensions that accounts for shear-induced particle migration. Physics of Fluids A, Vol. 4, No. 1, pp. 30-40, 1992.

ROMERO, O. J. Limite da Vazão Mínima do Processo de Revestimento por Extrusão de Soluções Poliméricas. $\mathrm{PhD}$ thesis, Pontifícia Universidade Católica do Rio de Janeiro, Rio de Janeiro, RJ, Brasil, 2003. 
ROMERO, O. J. et al. Low-flow limit in slot coating of dilute solutions of high molecular weight polymer. Journal of Non-Newtonian Fluid Mechanics, Vol. 118, No. 2-3, pp. 137-156, 2004.

RUSCHACK, K. J. Limiting flow in a pre-metering coating device. Chemical Engineering Science, Vol. 31, No. 11, pp. 1057-1060, 1976.

SAITO, H.; SCRIVEN, L. E. Study of Coating Flow by the Finite Element Method. Journal of Computational Physics, Vol. 42, No. 1, pp. 53-76, 1981.

SANI, R. L. et al. The cause and cure (?) of the spurious pressures generated by certain FEM solutions of the incompressible NavierStokes equations: Part 1, 2. International Journal for Numerical Methods in Fluids, Vol. 1, No. 1, pp. 17-43; No. 2, pp. 171-204, 1981.

SARTOR, L. Slot Coating: Fluid Mechanics and Die Design. PhD thesis, University of Minnesota, Minneapolis, MN, USA, 1990.

STICKEL, J. J.; POWELL, R. L. Fluid Mechanics and Rheology of Dense Suspensions. Annual Review of Fluid Mechanics, Vol. 45, pp. 129-149, 2005.

STOCKER, T. Introduction to Climate Modelling. Springer-Verlag Berlin Heidelberg 2011. 179p.

XI, C.; SHAPLEY, N. C. Flows of concentrated suspensions through an asymmetric bifurcation. Journal of Rheology, Vol. 52, No. 2, pp. 625647, 2008.

YEZAZ, A. G. M.; SINGH, A. Numerical simulation of particle migration in asymmetric bifurcation channel. Journal of Non-Newtonian Fluid Mechanics, Vol. 166, No. 1-2, pp. 42-51, 2011.

ZARRAGA, I. E.; HILL, D. A.; LEIGHTON, D. T. The characterization of the total stress of concentrated suspensions of noncolloidal spheres in Newtonian fluids. Journal of Rheology, Vol. 44, No. 2, pp. 185-220, 2000. 
ZHANG, K; ACRIVOS, A. Viscous resuspension in fully developed laminar pipe flows. International Journal of Multiphase Flow, Vol. 20, No. 3, pp. 579-591, 1994. 